\title{
EQUIVARIANT DEGENERATIONS OF SPHERICAL MODULES: PART II
}

\author{
STAVROS ARGYRIOS PAPADAKIS AND BART VAN STEIRTEGHEM
}

\begin{abstract}
We determine, under a certain assumption, the Alexeev-Brion moduli scheme $\mathrm{M}_{\mathcal{S}}$ of affine spherical $G$-varieties with a prescribed weight monoid $\mathcal{S}$. In [PVS12] we showed that if $G$ is a connected complex reductive group of type $\mathrm{A}$ and $\mathcal{S}$ is the weight monoid of a spherical G-module, then $\mathrm{M}_{\mathcal{S}}$ is an affine space. Here we prove that this remains true without any restriction on the type of $G$.
\end{abstract}

\section{INTRODUCTION AND STATEMENT OF RESULTS}

A natural invariant of an affine variety $X$ equipped with an action of a complex connected reductive group $G$ is its weight monoid $\mathcal{S}(X)$. It is the set of (isomorphism classes of irreducible representations of $G$ that occur in the ring of regular functions $\mathbb{C}[X]$. If every irreducible representation occurs at most once in this ring, then $X$ is called multiplicity-free. If, in addition, $X$ is normal, then it is an affine spherical variety. For multiplicity-free varieties, the weight monoid completely describes the structure of $\mathbb{C}[X]$ as a representation of $G$. Knop's Conjecture, proved by Losev in [Los09], asserts that if $X$ is smooth and multiplicity-free, then $\mathcal{S}(X)$ uniquely determines $X$. This is no longer true without the smoothness assumption. A moduli scheme introduced by V. Alexeev and M. Brion [AB05] brings geometry to the natural question, "to what extent does $\mathcal{S}(X)$ determine $X$ as a variety?"

To describe the moduli scheme, following [Bri13, Section 4.3], we introduce some more notation. Let $B$ be a Borel subgroup of $G$. Then $B=T U$ where $T$ is a maximal torus of $G$ and $U$ is the unipotent radical of $B$. Let $\Lambda^{+}$be the monoid of dominant weights in the weight lattice $\Lambda$. Recall that by highest weight theory, the elements of $\Lambda^{+}$are in bijection with the isomorphism classes of irreducible representations of $G$. Under this identification, the weight monoid $\mathcal{S}(X)$ of a multiplicity-free affine $G$-variety $X$ is a finitely generated submonoid of $\Lambda^{+}$. Now, given a finitely generated submonoid $\mathcal{S}$ of $\Lambda^{+}$, define the following G-module: $V(\mathcal{S}):=\oplus_{\lambda \in \mathcal{S}} V(\lambda)$. By identifying it with the semigroup algebra $\mathbb{C}[\mathcal{S}]$, we equip the space of highest weight vectors $V(\mathcal{S})^{U}$ with a $T$-multiplication law. The moduli scheme $\mathrm{M}_{\mathcal{S}}$ introduced in [AB05] parametrizes the $G$-multiplication laws on $V(\mathcal{S})$ that extend the chosen $T$-multiplication law on the subspace $V(\mathcal{S})^{U}$. We will sometimes write $\mathrm{M}_{\mathcal{S}}^{G}$ instead of $\mathrm{M}_{\mathcal{S}}$ when we need to specify the group under consideration. Alexeev and Brion showed that $\mathrm{M}_{\mathcal{S}}$ is an affine scheme of finite type over $\mathbb{C}$.

In more geometric language, the moduli scheme $\mathrm{M}_{\mathcal{S}}$ parametrizes pairs $(X, \varphi)$ where $X$ is a multiplicity-free affine $G$-variety with weight monoid $\mathcal{S}$ and $\varphi$ is a $T$-equivariant map $\operatorname{Spec}\left(\mathbb{C}[X]^{U}\right) \rightarrow \operatorname{Spec}(\mathbb{C}[\mathcal{S}])$. Alexeev and Brion equipped $\mathrm{M}_{\mathcal{S}}$ with a natural action of the 'adjoint torus' $T_{\mathrm{ad}}:=T / Z(G)$, where $Z(G)$ is the center of $G$. They proved that the orbits correspond to isomorphism classes of multiplicity-free affine varieties with weight monoid $\mathcal{S}$, and that there is a unique closed $T_{\text {ad }}$-orbit, which is a fixed point denoted $X_{0}$. 
Finally, they showed that if $X$ is an affine multiplicity-free variety with weight monoid $\mathcal{S}$, and we think of $X$ as a closed point on $\mathrm{M}_{\mathcal{S}}$, then the closure of the orbit $T_{\mathrm{ad}} \cdot X \subseteq \mathrm{M}_{\mathcal{S}}$ has coordinate ring $\mathbb{C}\left[\Sigma_{X}\right]$, where $\Sigma_{X}$ is the so-called root monoid of $X$ :

$$
\Sigma_{X}:=\left\langle\lambda+\mu-v: \lambda, \mu, v \in \Lambda^{+} \text {such that }\left\langle\mathbb{C}[X]_{(\lambda)} \cdot \mathbb{C}[X]_{(\mu)}\right\rangle_{\mathbb{C}} \cap \mathbb{C}[X]_{(v)} \neq 0\right\rangle_{\mathbb{N}} .
$$

Here $\mathbb{C}[X]_{(\lambda)}$ is the isotypic component of $\mathbb{C}[X]$ of type $\lambda \in \Lambda^{+}$.

1.1. Main results. In [PVS12] we proved that if $\mathcal{S}$ is the weight monoid of a spherical $G$-module, where $G$ is of type $A$, then $M_{\mathcal{S}}$ is an affine space. Here we extend this result to weight monoids of spherical $G$-modules for arbitrary connected reductive groups $G$. That is, we here prove the following.

Theorem 1.1. Assume $W$ is a spherical G-module, where $G$ is a connected reductive algebraic group. Let $\mathcal{S}$ be the weight monoid of $W$ and let $d_{W}$ be the rank of the group $\mathbb{Z} \Sigma_{W}$ generated by the root monoid $\Sigma_{W}$ of $W$. Then

(a) $\Sigma_{W}$ is a freely generated monoid; and

(b) the $T_{\mathrm{ad}}$-scheme $\mathrm{M}_{\mathcal{S}}$ is $T_{\mathrm{ad}}$-equivariantly isomorphic to the $T_{\mathrm{ad}}$-module with weight monoid $\Sigma_{W}$. In particular, the scheme $\mathrm{M}_{\mathcal{S}}$ is isomorphic to the affine space $\mathbb{A}^{d_{W}}$, hence it is irreducible and smooth.

We recall from [PVS12, Lemma 2.7] that for a given spherical G-module $W$, the invariant $d_{W}$ is easy to calculate from the rank of the free abelian group $\langle\mathcal{S}(W)\rangle_{\mathbb{Z}}$ : it is the difference between the rank of $\langle\mathcal{S}(W)\rangle_{\mathbb{Z}}$ and the number of irreducible components of $W$. Thanks to the reduction in Section 4 of [PVS12], which is independent of the type of the group G, the proof of Theorem 1.1, which is formally given in Section 1.2, reduces to the following theorem.

Theorem 1.2. Suppose $(\bar{G}, W)$ is an entry in Knop's List of saturated indecomposable spherical modules (see List 3.1] on page 16). If $G$ is a connected reductive group such that

(1) $\bar{G}^{\prime} \subseteq G \subseteq \bar{G}$; and

(2) $W$ is spherical as a G-module

then

$$
\operatorname{dim} T_{X_{0}} \mathbf{M}_{\mathcal{S}}^{G}=d_{W}
$$

where $\mathcal{S}$ is the weight monoid of $(G, W)$.

In [PVS12, Section 5], we proved Theorem 1.2 for groups $\bar{G}$ of type A. In Section 3 below, we prove it for the remaining modules in Knop's List, i.e. those where the acting group contains a component that is not of type A. As in our previous paper, we do this by determining for each entry in Knop's List the structure of $T_{X_{0}} \mathrm{M}_{\mathcal{S}}$ as a $T_{\mathrm{ad}}$-module: we determine the $T_{\mathrm{ad}}$-weights occuring in $T_{X_{0}} \mathrm{M}_{\mathcal{S}}$ and show that each weight has multiplicity one. It follows from our descriptions that only certain 'special' elements of the root lattice of $G$ occur as $T_{\text {ad }}$-weights in $T_{X_{0}} \mathrm{M}_{\mathcal{S}}$ : every $T_{\text {ad }}$-weight in $T_{X_{0}} \mathrm{M}_{\mathcal{S}}$ is a so-called "spherical root" of $G$ (cf. [Lun01, Section 1.2] for the definition of this notion).

Section 2, which may be of independent interest, contains some auxilary results about the tangent space $T_{X_{0}} \mathrm{M}_{\mathcal{S}}$ to $\mathrm{M}_{\mathcal{S}}$ at the point $X_{0}$. Corollary 2.9 is a sharpening of the extension criterion [PVS12, Proposition 3.4] for invariant sections of the normal sheaf of $X_{0}$ in $V$. 
The Appendix presents the details, in a specific case, of a different technique which explicitly computes the $T_{\text {ad }}$-eigenvectors in $\left(V / \mathfrak{g} \cdot x_{0}\right)^{G_{x_{0}}}$.

After most of the work on this paper had been completed, the preprints [ACF14] and [BVS15] were posted on the arXiv. We do not use the results contained in these papers, and for the weight monoids $\mathcal{S}$ under consideration in the present paper our main result is stronger. More precisely, while [ACF14, BVS15] also prove (and in much greater generality than in the present paper) that the $T_{\text {ad }}$-weights in $T_{X_{0}} \mathrm{M}_{\mathcal{S}}$ are spherical roots of $G$ and have multiplicity one, in the present paper we additionally prove that $\mathrm{M}_{\mathcal{S}}$ is irreducible for the monoids $\mathcal{S}$ under consideration.

1.2. Formal proof of Theorem 1.1. We now give the proof of Theorem 1.1. Corollary 2.6 and Corollary 4.17 of [PVS12] reduce the proof to Theorem 1.2, which we prove by a case-by-case verification in Section 3 ,

1.3. Notations. We will follow the conventions and notations of [PVS12]. In particular, by a variety we mean a reduced, irreducible and separated scheme of finite type over $\mathbb{C}$. We will use $\Lambda$ for the weight lattice of $G$, i.e. the group of characters of a fixed maximal torus $T$, which is identified with the group of characters of a chosen Borel subgroup $B$ of $G$ which contains $T$. Then $\Lambda^{+}$will denote the monoid of dominant weights in $\Lambda$, and we will use $V(\lambda)$ for the irreducible representation of $G$ corresponding to $\lambda \in \Lambda^{+}$, and $v_{\lambda}$ for a highest weight vector in $V(\lambda)$. We will use $\mathfrak{g}$ for the Lie algebra of $G$. If $\alpha$ is a root, then $\alpha^{\vee} \in \operatorname{Hom}_{\mathbb{Z}}(\Lambda, \mathbb{Z})$ will be its coroot (in the sense of [Bou68]), $\mathfrak{g}^{\alpha}$ its root space and $X_{\alpha} \in \mathfrak{g}^{\alpha} \backslash\{0\}$ a root operator. We will use $\Pi$ for the set of simple roots (relative to $T$ and $B$ ) and $\Lambda_{R}$ for the root lattice: $\Lambda_{R}=\langle\Pi\rangle_{\mathbb{Z}} \subseteq \Lambda$.

We will number the fundamental weights and the simple roots of the simple Lie algebras as in [Bou68]. When $G=\mathrm{GL}(n)$ and $i \in\{1, \ldots, n\}$, the highest weight of the module $\wedge^{i} \mathbb{C}^{n}$ will be denoted by $\omega_{i}$. Moreover, we put $\omega_{0}=0$. It is well-known that the simple roots of $\mathrm{GL}(n)$ have the following expressions in terms of the $\omega_{i}$ :

$$
\alpha_{i}=-\omega_{i-1}+2 \omega_{i}-\omega_{i+1} \quad \text { for } i \in\{1,2, \ldots, n-1\} .
$$

We will use $E^{*}$ for the basis of a free monoid $\mathcal{S}$ of dominant weights and $E:=\left\{\lambda^{*}: \lambda \in\right.$ $\left.E^{*}\right\}$. Here $\lambda^{*}$ is the highest weight of the representation $V(\lambda)^{*}$ which is dual to $V(\lambda)$; that is: $V\left(\lambda^{*}\right) \simeq V(\lambda)^{*}$.

1.4. Acknowledgment. The authors thank Michel Brion for suggesting, during a 2011 visit of the second-named author to the University of Grenoble, the general strategy for the extension criterion in Section 2, They also thank an anonymous referee for pointing out a mistake in the proof of Proposition 3.12, for her/his very careful reading and for the numerous and detailed suggestions which improved the paper.

S. P. benefited from experiments with the computer algebra program Macaulay2 [GS]. For different parts of the project he was financially supported by the Portuguese Fundação para a Ciência e a Tecnologia through grant SFRH/BPD/22846/2005 of POCI2010/FEDER and by RIMS, Kyoto University, Japan.

B. V. S. received support from The City University of New York PSC-CUNY Research Award Program and from the National Science Foundation through grant number DMS1407394. He also thanks Michel Brion and the Institut Fourier for their hospitality in the Summer of 2011. 


\section{CRITERION FOR EXTENSION OF SECTIONS}

In this section, $E^{*}$ is a set of linearly independent dominant weights of a complex connected reductive group $G$, and $\mathcal{S}$ is the submonoid of $\Lambda^{+}$generated by $E^{*}$. We do not assume that $\mathcal{S}$ is the weight monoid of a spherical module. Like before, $E=\left\{\lambda: \lambda^{*} \in E^{*}\right\}$. As in [PVS12], we put

$$
\begin{aligned}
V & :=\bigoplus_{\lambda \in E} V(\lambda) \\
x_{0} & :=\sum_{\lambda \in E} v_{\lambda} \in V ; \\
X_{0} & :=\overline{G \cdot x_{0}} \subseteq V
\end{aligned}
$$

and we denote by $\mathcal{N}_{X_{0} \mid V}$ the normal sheaf of $X_{0}$ in $V$.

Remark 2.1. We record some well-known facts about $x_{0}$ and $X_{0}$ that will be of use later in the paper.

(a) Since $E$ is linearly independent, $\mathfrak{t} \cdot x_{0}=\left\langle v_{\lambda}: \lambda \in E\right\rangle_{\mathbb{C}}$.

(b) $X_{0}$ is a spherical $G$-variety with weight monoid $\mathcal{S}$; cf. [VP72, Theorem 6]

(c) By [VP72, Theorem 8], the following map is a one-to-one correspondence between the set of subsets of $E$ and the set of $G$-orbits in $X_{0}$ :

$$
(D \subseteq E) \mapsto G \cdot v_{D} \quad \text { where } v_{D}:=\sum_{\lambda \in D} v_{\lambda}
$$

In [AB05] Alexeev and Brion equipped $\mathrm{M}_{\mathcal{S}}$ with an action of $T_{\mathrm{ad}}$ and showed that $X_{0}$, viewed as a point of $\mathrm{M}_{\mathcal{S}}$, is a fixed point and the unique closed orbit for this action. As in [PVS12] we will work with a 'twist' of the action in [AB05]. It is obtained by composing Alexeev and Brion's action with the automorphism of $T_{\text {ad }}$ induced by the automorphism $\gamma \mapsto$ $w_{0}(\gamma)$ of the root lattice $\Lambda_{R}$, which is the group of characters of $T_{\text {ad }}$. Here $w_{0}$ is the longest element of the Weyl group of $(G, T)$. We will call our action on $\mathrm{M}_{\mathcal{S}}$ and its induced action on $T_{X_{0}} \mathrm{M}_{\mathcal{S}}$ "the $T_{\mathrm{ad}}$-action." As shown in [AB05] and reviewed in [PVS12, §2.2], we have a sequence of $T_{\text {ad }}$-equivariant linear maps

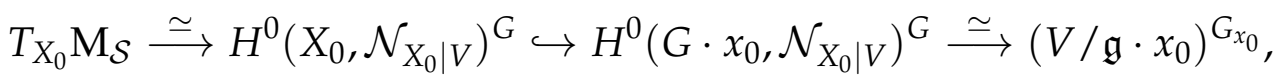

where the first and the third map are isomorphisms, and the second one is an inclusion.

Because they will play a role later on, we recall from [PVS12, §2.2] explicit descriptions of our $T_{\text {ad }}$-actions on $\left(V / \mathfrak{g} \cdot x_{0}\right)^{G_{x_{0}}}$ and on $H^{0}\left(X_{0}, \mathcal{N}_{X_{0} \mid V}\right)^{G}$. For the former, we begin by equipping $V$ with the same action $\alpha$ of $T_{\text {ad }}$ as in [PVS12, Definition 2.11]: if $t \in T, \lambda \in E$ and $v \in V(\lambda) \subseteq V$ then

$$
\alpha(t, v)=\lambda(t) t^{-1} v
$$

It follows from highest weight theory that the center $Z(G)$ of $G$ belongs to the kernel of $\alpha$, and therefore $\alpha$ induces an action of $T_{\mathrm{ad}}=T / Z(G)$ on $V$. Let $G \rtimes T_{\mathrm{ad}}$ be the semidirect product of $G$ and $T_{\mathrm{ad}}$, where $T_{\mathrm{ad}}$ acts on $G$ by conjugation. As explained in [AB05, p. 102] the $T_{\text {ad }}$-action $\alpha$ and the linear $G$-action on $V$ can be extended together to a linear action of $G \rtimes T_{\text {ad }}$ on $V$. Since the $T_{\text {ad }}$-action fixes $x_{0}$, this yields an action of $G_{x_{0}} \rtimes T_{\text {ad }}$ on $V / \mathfrak{g} \cdot x_{0}$, see e.g. [PVS12, p. 1780]. It follows that the subspace $\left(V / \mathfrak{g} \cdot x_{0}\right)^{G_{x_{0}}}$ of $V / \mathfrak{g} \cdot x_{0}$ is preserved 
by the action of $T_{\text {ad }}$. This induced action on $\left(V / \mathfrak{g} \cdot x_{0}\right)^{G_{x_{0}}}$ is what we call "the $T_{\text {ad }}$-action" on $\left(V / \mathfrak{g} \cdot x_{0}\right)^{G_{x_{0}}}$. By slight abuse of notation, we also denote it by $\alpha$.

To describe the $T_{\text {ad }}$-action on $H^{0}\left(G \cdot x_{0}, \mathcal{N}_{X_{0} \mid V}\right)^{G}$ and on $H^{0}\left(X_{0}, \mathcal{N}_{X_{0} \mid V}\right)^{G}$, let $\operatorname{GL}(V)^{G}$ be the group of linear automorphisms of $V$ that commute with the action of $G$. Since the elements of $E$ are distinct, $\operatorname{GL}(V)^{G}$ is isomorphic to the product of $|E|$ copies of $\mathbb{C}^{\times}$. The natural action of $\mathrm{GL}(V)^{G}$ on $V$ stabilizes $G \cdot x_{0}$ and $X_{0}$ and the embedding $H^{0}\left(X_{0}, \mathcal{N}_{X_{0} \mid V}\right)^{G} \hookrightarrow H^{0}\left(G \cdot x_{0}, \mathcal{N}_{X_{0} \mid V}\right)^{G}$ is $\mathrm{GL}(V)^{G}$-equivariant for the induced actions. Composing the action of $\mathrm{GL}(V)^{G}$ with the homomorphism

$$
f: T \rightarrow \mathrm{GL}(V)^{G}, t \mapsto(\lambda(t))_{\lambda \in E}
$$

yields an action of $T$ on $V$. We denote the induced $T$-action on $H^{0}\left(G \cdot x_{0}, \mathcal{N}_{X_{0} \mid V}\right)^{G}$ and on $H^{0}\left(X_{0}, \mathcal{N}_{X_{0} \mid V}\right)^{G}$ by $\widehat{\psi}$. Proposition 2.13 of [PVS12] shows that $Z(G)$ is in the kernel of $\widehat{\psi}$ and that the isomorphism

$$
H^{0}\left(G \cdot x_{0}, \mathcal{N}_{X_{0} \mid V}\right)^{G} \rightarrow\left(V / \mathfrak{g} \cdot x_{0}\right)^{G_{x_{0}}, s} \mapsto s\left(x_{0}\right)
$$

in (2.1) above is indeed $T_{\text {ad }}$-equivariant if $\left(V / \mathfrak{g} \cdot x_{0}\right)^{G_{x_{0}}}$ is equipped with the $T_{\text {ad }}$-action $\alpha$ and $H^{0}\left(G \cdot x_{0}, \mathcal{N}_{X_{0} \mid V}\right)^{G}$ is equipped with the $T_{\text {ad }}$-action $\widehat{\psi}$.

In Section 2.1 we strengthen [PVS12, Proposition 3.4] and obtain necessary and sufficient conditions for a section $s \in H^{0}\left(G \cdot x_{0}, \mathcal{N}_{X_{0} \mid V}\right)^{G}$ to extend to $X_{0}$ : see Corollary 2.9. The proof is given in Section [2.3, after we review some generalities about extending sections of a vector bundle over a normal variety in Section 2.2. In Section 2.4 we gather a few more results on $T_{X_{0}} \mathrm{M}_{\mathcal{S}}$.

2.1. Extending sections. We denote by $X_{0}^{\leq 1} \subset X_{0}$ the union of $G \cdot x_{0}$ with all $G$-orbits of $X_{0}$ that have codimension 1. By [Bri10, Lemma 1.14] $X_{0}^{\leq 1}$ is an open subset of $X_{0}$, and because $X_{0}$ is normal, it is a subset of the smooth locus of $X_{0}$ (see, e.g., the argument in the proof of [PVS12, Lemma 3.3] for details).

Definition 2.2. We say the $\lambda \in E$ has codimension one if

$$
\operatorname{dim} G \cdot\left(x_{0}-v_{\lambda}\right)=\left(\operatorname{dim} G \cdot x_{0}\right)-1 .
$$

As an immediate consequence of, e.g., [PVS12, Proposition 3.1] one has the following simple criterion to determine whether an element of $E$ has codimension one.

Proposition 2.3. For $\lambda \in E$ the following are equivalent

(1) $\lambda$ has codimension one;

(2) for every $\alpha \in \Pi$ such that $\left\langle\alpha^{\vee}, \lambda\right\rangle \neq 0$, there exists $\mu \in E \backslash\{\lambda\}$ such that $\left\langle\alpha^{\vee}, \mu\right\rangle \neq 0$;

(3) for every positive root $\alpha$ such that $\left\langle\alpha^{\vee}, \lambda\right\rangle \neq 0$, there exists $\mu \in E \backslash\{\lambda\}$ such that $\left\langle\alpha^{\vee}, \mu\right\rangle \neq 0$.

The following is an immediate consequence of [Bri13, Lemma 3.9].

Proposition 2.4. If $s \in H^{0}\left(G \cdot x_{0}, \mathcal{N}_{X_{0} \mid V}\right)^{G}$, then the following are equivalent:

(1) s extends to $X_{0}$;

(2) s extends to $X_{0}^{\leq 1}$;

(3) s extends to $G \cdot x_{0} \cup G \cdot\left(x_{0}-v_{\lambda}\right)$ for every $\lambda \in E$ of codimension 1 . 
Proof. The equivalence of (1) and (2) is a special case of [Bri13, Lemma 3.9]. The equivalence of (2) and (3) is a consequence of the definition of a sheaf, once we prove that the collection of sets

$$
\left\{G \cdot x_{0} \cup G \cdot\left(x_{0}-v_{\lambda}\right): \lambda \in E \text { of codimension } 1\right\}
$$

forms an open cover of $X_{0}^{\leq 1}$. We first show that each set $G \cdot x_{0} \cup G \cdot\left(x_{0}-v_{\lambda}\right)$ in the collection (2.5) is open. Indeed, the complement of $G \cdot x_{0} \cup G \cdot\left(x_{0}-v_{\lambda}\right)$ in $X_{0}^{\leq 1}$ is a finite (by Remark 2.1(C) ) union of orbits in $X_{0}^{\leq 1}$ that are all closed because they are of minimal dimension. Secondly, the union of the sets in the collection (2.5) is all of $X_{0}^{\leq 1}$ because, by Remark[2.1(C) and [PVS12, Lemma 2.16] every orbit of codimension 1 in $X_{0}$ is of the form $G \cdot\left(x_{0}-v_{\lambda}\right)$ for some $\lambda \in E$ of codimension 1 .

We recall some well-known facts about $T_{\text {ad }}$-weights and $T_{\text {ad }}$-eigenvectors in $(V / \mathfrak{g}$. $\left.x_{0}\right)^{G_{x_{0}}}$.

Lemma 2.5. Let $\beta \in \Lambda$. If $v \in V$ such that $[v] \in\left(V / \mathfrak{g} \cdot x_{0}\right)^{G_{x_{0}}}$ is a nonzero $T_{\text {ad-eigenvector of }}$ $T_{\mathrm{ad}}$-weight $\beta$, then there exists a $T_{\mathrm{ad}}$-eigenvector $\widehat{v} \in V$ of $T_{\mathrm{ad}}$-weight $\beta$ such that $[v]=[\widehat{v}] \in$ $\left(V / \mathfrak{g} \cdot x_{0}\right)^{G_{x_{0}}}$.

Proof. This follows from the following standard argument. Note that $\left(V / \mathfrak{g} \cdot x_{0}\right)^{G_{x_{0}}}$ is a

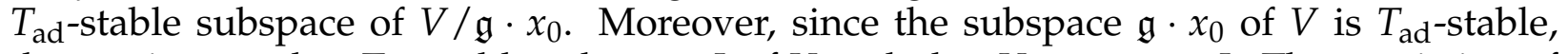
there exists another $T_{\text {ad }}$-stable subspace $L$ of $V$ such that $V=\mathfrak{g} \cdot x_{0} \oplus L$. The restriction of the quotient map $V \rightarrow V / \mathfrak{g} \cdot x_{0}$ to $L$ is an isomorphism $L \rightarrow V / \mathfrak{g} \cdot x_{0}$ of $T_{\text {ad }}$-modules. We can take $\widehat{v}$ to be the inverse image in $L$ of $[v]$ under this isomorphism.

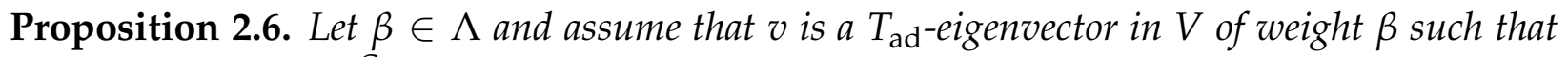
$0 \neq[v] \in\left(V / \mathfrak{g} \cdot x_{0}\right)^{G_{x_{0}}}$. Then

(a) there exists $\alpha \in \Pi$ such that $X_{\alpha} \cdot v \neq 0$ and $\beta-\alpha \in R^{+} \cup\{0\}$;

(b) $\beta \in\langle E\rangle_{\mathbb{Z}}$;

(c) $X_{\alpha} \cdot v \in\left\langle X_{-(\beta-\alpha)} \cdot x_{0}\right\rangle_{C}$ for all $\alpha \in \Pi$ such that $\beta-\alpha \in R^{+}$;

(d) $X_{\alpha} \cdot v=0$ for all $\alpha \in \Pi$ such that $\beta-\alpha \notin R^{+} \cup\{0\}$.

Proof. Assertion (b) is a consequence in Lemma 2.17(c) of [PVS12]. Let $\alpha \in \Pi$. Recalling that if $X_{\alpha} \cdot v$ is nonzero, then it has $T_{\text {ad }}$-weight $\beta-\alpha$, assertions (a) and (d) follow from Lemma 2.18 in loc.cit. Since the root operator $X_{\alpha}$ belongs to the Lie algebra of $G_{x_{0}}$, we have that $X_{\alpha} \cdot v \in \mathfrak{g} \cdot x_{0}$. Assertion (ㄷ) now follows from the fact that if the $T_{\text {ad }}$-weight $\beta-\alpha$ occurs in $\mathfrak{g} \cdot x_{0}$ then the corresponding weight space is $\left\langle X_{-(\beta-\alpha)} \cdot x_{0}\right\rangle_{\mathbb{C}}$.

Remark 2.7. Since we will frequently make use of it later, we note the following consequence of (a) and (b) in Proposition 2.6 if $\beta$ is a $T_{\text {ad }}$-weight in $\left(V / \mathfrak{g} \cdot x_{0}\right)^{G_{x_{0}}}$ then

$$
0 \neq \beta \in\langle\Pi\rangle_{\mathbb{N}} \cap\langle E\rangle_{\mathbb{Z}}
$$

Theorem 2.8. Assume that $v$ is a $T_{\mathrm{ad}}$-eigenvector in $V$ of $T_{\mathrm{ad}}$-weight $\beta$ such that

$$
0 \neq[v] \in\left(V / \mathfrak{g} \cdot x_{0}\right)^{G_{x_{0}}} .
$$

Denote by $s \in H^{0}\left(G \cdot x_{0}, \mathcal{N}_{X_{0} \mid V}\right)^{G}$ the G-equivariant section defined by $s\left(x_{0}\right)=[v]$. Let $\lambda$ be an element of $E$ which has codimension 1 and put $Z=G \cdot x_{0} \cup G \cdot\left(x_{0}-v_{\lambda}\right)$. Let a be the coefficient of $\lambda$ in the unique expression of $\beta$ as a linear combination of elements of $E$. 
A) If $a \leq 0$, then s extends to an element of $H^{0}\left(Z, \mathcal{N}_{X_{0} \mid V}\right)^{G}$.

B) If $a>1$, then $s$ does not extend to an element of $H^{0}\left(Z, \mathcal{N}_{X_{0} \mid V}\right)^{G}$.

C) Assume $a=1$. Then the following are equivalent:

i) sextends to an element of $H^{0}\left(Z, \mathcal{N}_{X_{0} \mid V}\right)^{G}$;

ii) There exists $\hat{v} \in V(\lambda)$ such that $[v]=[\hat{v}]$ as elements of $V / \mathfrak{g} \cdot x_{0}$.

The proof of Theorem 2.8 will be given in Section 2.3 which starts on page 10. Before that, we gather some general results on extending sections in Section 2.2. The following is a synthesis of Proposition 2.4 and Theorem 2.8.

Corollary 2.9. Assume that $v$ is a $T_{\text {ad }}$-eigenvector in $V$ of $T_{\text {ad }}$-weight $\beta$ such that

$$
0 \neq[v] \in\left(V / \mathfrak{g} \cdot x_{0}\right)^{G_{x_{0}}} .
$$

Denote by $s \in H^{0}\left(G \cdot x_{0}, \mathcal{N}_{X_{0} \mid V}\right)^{G}$ the G-equivariant section defined by $s\left(x_{0}\right)=[v]$. Let

$$
\beta=\sum_{\lambda \in E} a_{\lambda} \lambda
$$

be the unique expression of $\beta$ as a $\mathbb{Z}$-linear combination of the elements of $E$.

The section sextends to an element of $H^{0}\left(X_{0}, \mathcal{N}_{X_{0} \mid V}\right)^{G}$ if and only if for all $\lambda \in E$ of codimension 1 we have

$-a_{\lambda} \leq 1 ;$ and

- if $a_{\lambda}=1$ then there exists $\hat{v} \in V(\lambda)$ such that $[v]=[\hat{v}]$ as elements of $V / \mathfrak{g} \cdot x_{0}$.

Remark 2.10. It follows from Proposition 2.20 below that if $s$ extends, then at most two of the $a_{\lambda}$ in equation 2.7 are positive, irrespective of whether $\lambda$ is of codimension 1 or not.

Example 2.11 (Luna). Let $G=\mathrm{SL}_{2} \times \mathrm{SL}_{2}$, and $E=\left\{\lambda_{1}, \lambda_{2}\right\}$ with $\lambda_{1}=2 \omega, \lambda_{2}=4 \omega+$ $2 \omega^{\prime}$. Here $\omega$ is the fundamental weight of the first component of $G$, and $\omega^{\prime}$ is that of the second component. Similarly, we will use $\alpha$ and $\alpha^{\prime}$ for the simple root of the first and second component of $G$, respectively. Using Proposition 2.3, it follows that $v_{\lambda_{2}}$ has a $G$-orbit of codimension 1 in $X_{0}$, while $v_{\lambda_{1}}$ has a $G$-orbit of codimension $\geq 2$. Hence by Proposition 2.4 for $[w] \in\left(V / \mathfrak{g} \cdot x_{0}\right)^{G_{x_{0}}}$, the equivariant section of $\mathcal{N}_{X_{0} \mid V}$ on $G \cdot x_{0}$ induced by $[w]$ extends to $X_{0}$ if and only if it extends over $G \cdot x_{0} \cup G \cdot v_{\lambda_{2}}$.

Denote by $e_{1}, e_{2}$ (resp. $g_{1}, g_{2}$ ) a basis of $\mathbb{C}^{2}$ where the first (resp. second) $\mathrm{SL}_{2}$ acts in the standard fashion. A small calculation gives that the vector space $\left(V / \mathfrak{g} \cdot x_{0}\right)^{G_{x_{0}}}$ is 3dimensional with basis the classes in $V / \mathfrak{g} \cdot x_{0}$ of

$$
w_{1}=e_{1} e_{2}, \quad w_{2}=e_{1}^{4} \otimes g_{2}^{2}, \quad w_{3}=e_{2}^{2}+e_{1}^{2} e_{2}^{2} \otimes g_{1}^{2} .
$$

The vector $w_{1}$ has $T_{\text {ad }}$ weight $\alpha=\lambda_{1}$, and since $w_{1} \in V\left(\lambda_{1}\right)$ part C) of Theorem 2.8 implies that the induced equivariant section extends to $G \cdot x_{0} \cup G \cdot v_{\lambda_{2}}$, hence to the whole of $X_{0}$. The vector $w_{2}$ has $T_{\text {ad }}$ weight $2 \alpha^{\prime}=-4 \lambda_{1}+2 \lambda_{2}$, and part $\mathrm{A}$ ) of Theorem 2.8 implies that the induced equivariant section extends to $X_{0}$. The vector $w_{3}$ has $T_{\text {ad }}$ weight $2 \alpha=2 \lambda_{1}$, hence part B) of Theorem 2.8 implies that the induced equivariant section does not extend to $G \cdot x_{0} \cup G \cdot v_{\lambda_{2}}$. We have shown that

$$
T_{X_{0}} \mathrm{M}_{\mathcal{S}}=\underset{7}{V(\alpha)} \oplus V\left(2 \alpha^{\prime}\right)
$$


as a $T_{\text {ad }}$-module. We remark that to exclude the section induced by $w_{3}$ from $T_{X_{0}} \mathrm{M}_{\mathcal{S}}$ we could not have used [PVS12, Proposition 3.4], since condition (ES2) of that proposition is not satisfied for $w_{3}$.

We also remark that Luna has shown in an unpublished note from 2005 that this moduli scheme $\mathrm{M}_{\mathcal{S}}$, equipped with its reduced scheme structure, is a union of two affine lines meeting in a point. It was the first example of a non-irreducible scheme $\mathrm{M}_{\mathcal{S}}$.

2.2. Generalities about extending sections. In this section $X$ denotes a variety, in particular it is reduced, irreducible and separated. Let $\mathcal{E} \rightarrow X$ be an algebraic vector bundle. For the proof of Theorem 2.8 in Section 2.3 we need the following general propositions. They are well known, but for completeness we provide proofs.

Proposition 2.12. Assume that $X$ is normal, that $U \subseteq X$ is a nonempty Zariski open subset, and that $f: U \rightarrow \mathbb{C}$ a morphism. If $f$ does not extend to a morphism $X \rightarrow \mathbb{C}$, then there exists $p \in X \backslash U$ such that for every irreducible algebraic curve $C \subseteq X$ with $p \in C$ and $U \cap C \neq \varnothing$ the morphism $f$ restricted to $U \cap C$ does not extend to a morphism $C \rightarrow \mathbb{C}$.

Proof. We consider $f$ as a rational function on $X$. Since $f$ does not extend to a morphism $X \rightarrow \mathbb{C}$ it follows that $f$ is not the constant function with value 0 . Using that $X$ is normal, there is a well defined divisor of poles of $f$ and a well defined divisor of zeroes of $f$, see, e.g. [CLS11, Section 4.0]. Since $f$ does not extend to a morphism $X \rightarrow \mathbb{C}$ the divisor of poles of $f$ is nonzero, see, e.g. [CLS11, Propopsition 4.0.16].

We fix a point $p \in X$ which is in the support of the divisor of poles of $f$ but not in the support of the divisor of zeroes. To obtain a contradiction, we assume that there exists an irreducible algebraic curve $C \subset X$ with $p \in C$ and $U \cap C \neq \varnothing$ such that the morphism $f$ restricted to $U \cap C$ extends to a morphism $\bar{f}: C \rightarrow \mathbb{C}$.

Denote by $g$ the rational function $1 / f$. The divisor of zeroes of $g$ is the divisor of poles of $f$ and the divisor of poles of $g$ is the divisor of zeroes of $f$. Hence $p$ is in the support of the divisor of zeroes of $g$ and is not in the support on the divisor of poles of $g$. It follows that there exists a Zariski open subset $W \subset X$ with $p \in W$ such that $g$ defines a morphism $g: W \rightarrow \mathbb{C}$ with the property $g(p)=0$. Denote by $\bar{g}: W \cap C \rightarrow \mathbb{C}$ the restriction of $g$ to $W \cap C$. We have that $W \cap U$ is a nonempty Zariski open subset of $X$. Since $W \cap C \neq \varnothing$ and $U \cap C \neq \varnothing$ we get that $W \cap U \cap C \neq \varnothing$. For $q \in W \cap U \cap C$ we have $(\bar{f} \bar{g})(q)=(f g)(q)=1$. Since $W \cap U \cap C$ is dense in $C$ it follows that $(\bar{f} \bar{g})(p)=1$ which contradicts $\bar{g}(p)=0$.

Example 2.13. If $X=\mathbb{C}^{2}$ and $f=x / y$ we can choose $p=(a, 0)$ for any $a \in \mathbb{C} \backslash\{0\}$.

Proposition 2.14. Assume that $X$ is normal, that $U \subset X$ is a nonempty Zariski open subset, and that $s \in H^{0}(U, \mathcal{E})$ is a section of the vector bundle $\mathcal{E}$. If s does not extend to a section $X \rightarrow \mathcal{E}$, then there exists $p \in X \backslash U$ such that for every algebraic curve $C \subset X$ with $p \in C$ and $U \cap C \neq \varnothing$ the section s restricted to $U \cap C$ does not extend to a section $C \rightarrow \mathcal{E}$.

Proof. By the defining gluing property of sections of sheaves, there exists a nonempty Zariski open $V \subset X$ such that $\mathcal{E}$ restricted to $V$ is trivial and $s$ restricted to $V \cap U$ does not extend to a section over $V$. Hence we can assume, without loss of generality, that $\mathcal{E}$ is the trivial vector bundle. So assume $\mathcal{E}=X \times \mathbb{C}^{n} \rightarrow X$ is the first projection. Let $e_{1}, \ldots, e_{n}$ be a basis of $\mathbb{C}^{n}$ and define $s_{i} \in H^{0}(X, \mathcal{E})$ by $s_{i}(x)=\left(x, e_{i}\right)$ for all $x \in X$. There exist (unique) 
morphisms $f_{i}: U \rightarrow \mathbb{C}$ such that

$$
s(u)=\sum_{i=1}^{n} f_{i}(u) s_{i}(u)
$$

for all $u \in U$. If each $f_{i}$ extended to a morphism $X \rightarrow \mathbb{C}$, it would then follow that $s$ extends to a section $X \rightarrow \mathcal{E}$ which contradicts the assumptions. Hence at least one of the $f_{i}$ does not extend to a morphism. Using Proposition 2.12 there exists $p \in X \backslash U$ such that for every algebraic curve $C \subset X$ with $p \in C$ and $U \cap C \neq \varnothing$, the morphism $f_{i}$ restricted to $U \cap C$ does not extend to a morphism $C \rightarrow \mathbb{C}$. As a consequence, $s$ restricted to $U \cap C$ does not extend to a section $C \rightarrow \mathcal{E}$.

Assume now in addition that $G$ is a connected linear algebraic group over $\mathbb{C}, X$ is a $G$-variety and $\pi: \mathcal{E} \rightarrow X$ is a $G$-vector bundle over $X$. This means that we are given an algebraic action $\rho: G \times \mathcal{E} \rightarrow \mathcal{E}$ such that

$$
\rho(g, v) \in \pi^{-1}(g \cdot(\pi(v)))
$$

for all $g \in G, v \in V$ and that for fixed $g \in G$ and $x \in X$ the induced map

$$
\pi^{-1}(x) \rightarrow \pi^{-1}(g \cdot x), \quad v \mapsto \rho(g, v)
$$

is an isomorphism of vector spaces.

While its proof is elementary, the following proposition implies that the section $s$ of Theorem 2.8 extends to $Z$ if and only if it extends along just one curve; see Proposition 2.16.

Proposition 2.15. Assume that $X$ is normal, that $U \subset X$ is a nonempty $G$-stable Zariski open subset such that $X \backslash U$ is a single G-orbit, and that $s \in H^{0}(U, \mathcal{E})^{G}$ is a G-equivariant section $U \rightarrow \mathcal{E}$. Assume that there exists $p_{0} \in X \backslash U$ and an algebraic curve $C_{0} \subset X$ with $p_{0} \in C_{0}$ and $U \cap C_{0} \neq \varnothing$ such that s restricted to $U \cap C_{0}$ extends to a section $s_{0}: C_{0} \rightarrow \mathcal{E}$. Then s extends to an element of $H^{0}(X, \mathcal{E})^{G}$.

Proof. We first show that $s$ extends to an element of $H^{0}(X, \mathcal{E})$, and then that the extension is G-equivariant.

We assume that $s$ does not extend to an element of $H^{0}(X, \mathcal{E})$, and we will get a contradiction. By Proposition 2.14 there exists $p \in X \backslash U$ such that for every algebraic curve $C \subset X$ with $p \in C$ and $U \cap C \neq \varnothing$ the section $s$ restricted to $U \cap C$ does not extend to a section $C \rightarrow \mathcal{E}$. Since $X \backslash U$ is a single $G$-orbit, there exists $g \in G$ with $g \cdot p_{0}=p$.

Set $C=\left\{g \cdot v: v \in C_{0}\right\}$ and define $t: C \rightarrow \mathcal{E}$ by

$$
t(v)=g \cdot s_{0}\left(g^{-1} \cdot v\right)
$$

for $v \in C$. Since $s$ is $G$-equivariant we have $\left.t\right|_{U \cap C}=\left.s\right|_{U \cap C}$, hence $t$ is a section of $\mathcal{E}$ over $C$ which extends $\left.s\right|_{U \cap C}$, contradicting the choice of $p$.

We have shown that $s$ extends to a section $s_{1}: X \rightarrow \mathcal{E}$. We claim that $s_{1}$ is $G$-equivariant. Indeed, define $s_{2}: X \rightarrow \mathcal{E}$ by $s_{2}(v)=g \cdot s_{1}\left(g^{-1} \cdot v\right)$. Since $s$ is $G$-equivariant on $U$, we have that $s_{2}(u)=s(u)=s_{1}(u)$ for all $u \in U$. As a consequence $s_{2}=s_{1}$, which implies that $s_{1}$ is $G$-equivariant. 
2.3. Proof of Theorem 2.8, We start the proof of Theorem 2.8, Let $\lambda \in E$ be of codimension 1 . For $t \in \mathbb{C}$, we put

$$
z_{t}:=t \cdot v_{\lambda}+\sum_{\mu \in E \backslash\{\lambda\}} v_{\mu} .
$$

Note that, because $E$ is linearly independent, $z_{t} \in T \cdot x_{0} \subseteq G \cdot x_{0}$ for $t \in \mathbb{C} \backslash\{0\}$. Moreover,

$$
z_{0}=\sum_{\mu \in E \backslash\{\lambda\}} v_{\mu}=x_{0}-v_{\lambda} ;
$$

and $G \cdot z_{0}$ has codimension 1 in $X_{0}$.

Since $Z=G \cdot x_{0} \cup G \cdot z_{0}$ is smooth, the restriction of the sheaf $\mathcal{N}_{X_{0} \mid V}$ to $Z$ is locally free. We denote by $\mathcal{E} \rightarrow Z$ the total space of the corresponding vector bundle. In particular the sections of the restriction of $\mathcal{N}_{X_{0} \mid V}$ to $Z$ are naturally identified with those of $\mathcal{E}$.

Recall that $s \in H^{0}\left(G \cdot x_{0}, \mathcal{E}\right)^{G}$ denotes the equivariant section induced by $v$; that is $s\left(x_{0}\right)=[v] \in\left(V / \mathfrak{g} \cdot x_{0}\right)^{G_{x_{0}}}$. Set $C_{0}=\left\{z_{t}: t \in \mathbb{C}\right\}$, and denote by $s^{*}$ the section of $\mathcal{E}$ over $G \cdot x_{0} \cap C_{0}$ defined by $s^{*}\left(z_{t}\right)=s\left(z_{t}\right)$.

Proposition 2.16. The section s extends to an element of $H^{0}(Z, \mathcal{E})^{G}$ if and only if $s^{*}$ extends to a section of $\mathcal{E}$ over $C_{0}$.

Proof. If $s^{*}$ extends, then so does $s$ by Proposition 2.15, The converse is obvious.

For $w \in V$ we denote by $s_{w} \in H^{0}\left(X_{0}, \mathcal{N}_{X_{0} \mid V}\right)$ the global section defined by

$$
s_{w}(x)=[w] \in V / T_{x} X_{0}
$$

for all $x \in X_{0}$. We will use $V^{\beta}$ for the $T_{\text {ad }}$-weight space in $V$ of weight $\beta$. Recall that, by Proposition 2.6, $\beta$ is a nonzero element of $\langle\Pi\rangle_{\mathbb{N}}$ and that $v \in V^{\beta}$ such that $0 \neq[v] \in$ $\left(V / \mathfrak{g} \cdot x_{0}\right)^{G_{x_{0}}}$.

The idea of the proof of Theorem 2.8 is to find elements $\left\{y_{i}\right\}$ in $V^{\beta}$ such that their images in $V / T_{z_{t}} X_{0}$ form a basis of the $T_{\text {ad }}$-weight space of weight $\beta$ in $V / T_{z_{t}} X_{0}$ for all $t \in \mathbb{C}$. It then follows that (the restriction to $C_{0}$ of) the sections $s_{y_{i}}$ form a linearly independent subset of $H^{0}\left(C_{0}, \mathcal{E}\right)$, and that there exist $f_{i} \in \mathbb{C}(t)$ such that for all $t \in \mathbb{C} \backslash\{0\}$ we have

$$
s^{*}\left(z_{t}\right)=\sum_{i} f_{i}(t) s_{y_{i}}\left(z_{t}\right) .
$$

The section $s^{*}$ extends to all of $C_{0}$ if and only if each $f_{i}(t)$ belongs to the polynomial ring $\mathbb{C}[t]$. With the appropriate choice of the vectors $\left\{y_{i}\right\}$ the rational functions $f_{i}(t)$ are very simple; see Proposition 2.18,

By [PVS12, Lemma 3.3] $T_{z_{0}} X_{0}$ is the linear span of $\mathfrak{g} \cdot z_{0} \cup\left\{v_{\lambda}\right\}$. If $t$ is nonzero, then $G \cdot z_{t}$ is open in $X_{0}$, whence $T_{z_{t}} X_{0}=\mathfrak{g} \cdot z_{t}$, and $v_{\lambda} \in T_{z_{t}} X_{0}$, by Remark 2.1(苘). The image of $V^{\beta}$ under the projection $V \rightarrow V / T_{z_{t}} X_{0}$ can naturally be identified with $V^{\beta} /\left(V^{\beta} \cap T_{z_{t}} X_{0}\right)$.

Lemma 2.17. Assume $t \in \mathbb{C}$. If $\beta$ is not a root, then $V^{\beta} \cap T_{z_{t}} X_{0}=0$. If $\beta$ is a root, then $V^{\beta} \cap T_{z_{t}} X_{0}$ is equal to $\left\langle X_{-\beta} \cdot z_{t}\right\rangle_{\mathbb{C}}$, so it is either 0 or 1 -dimensional.

Proof. Recall that $T_{z_{t}} X_{0}$ is equal to the linear span of $\mathfrak{g} \cdot z_{t} \cup\left\{v_{\lambda}\right\}$. Using that $\mathfrak{u}^{-}$is spanned by the set $\left\{X_{-\gamma}: \gamma\right.$ positive root of $\left.G\right\}$, the lemma follows from the fact that $v_{\lambda}$ has $T_{\text {ad }^{-}}$ weight zero (and so not equal to $\beta$ ) and that $\mathfrak{g} \cdot z_{t}=\mathfrak{t} \cdot z_{t} \oplus \mathfrak{u}^{-} \cdot z_{t}$. 
Before giving the details of the remaining arguments for the proof of Theorem 2.8 , we introduce some more notation for the remainder of this section. Put

$$
V_{1}:=V(\lambda) ; \quad V_{2}:=\oplus_{\mu \in E \backslash\{\lambda\}} V(\mu) .
$$

Note that $V=V_{1} \oplus V_{2}$. Set $n=\operatorname{dim} V^{\beta}+1$ and $m=n-\operatorname{dim} V^{\beta} \cap V_{1}$.

Because the summands $V_{1}$ and $V_{2}$ of $V$ are stable under the $T_{\text {ad }}$-action, there exists a basis $y_{2}, \ldots, y_{n}$ of $V^{\beta}$, such that $y_{i} \in V^{\beta} \cap V_{2}$ for $2 \leq i \leq m$ and $y_{i} \in V^{\beta} \cap V_{1}$ for $m+1 \leq i \leq n$. Since $v \in V^{\beta}$, there exist $b_{i} \in \mathbb{C}$ such that

$$
v=\sum_{i=2}^{n} b_{i} y_{i}
$$

Recall that $a$ is the coefficient of $\lambda$ in the unique expression of $\beta$ as a linear combination of the elements of $E$.

Proposition 2.18. Let $\left\{y_{2}, y_{3}, \ldots, y_{n}\right\}$ be a basis of $V^{\beta}$ as above, and let $b_{i}$ be elements of $\mathbb{C}$ such that the equality (2.11) holds. For all $t \in \mathbb{C}^{*}$ we have

$$
s\left(z_{t}\right)=t^{-a}\left(\sum_{i=2}^{m} b_{i} s_{y_{i}}\left(z_{t}\right)\right)+t^{-a+1}\left(\sum_{i=m+1}^{n} b_{i} s_{y_{i}}\left(z_{t}\right)\right) .
$$

Proof. By assumption, $s$ is an eigenvector of weight $\beta$ for the $T_{\text {ad }}$-action $\widehat{\psi}$ on $H^{0}(G$. $\left.x_{0}, \mathcal{E}\right)^{G} \cong\left(V / \mathfrak{g} \cdot x_{0}\right)^{G_{x_{0}}}$. Equivalently, $s$ is an eigenvector (of weight described below) for the natural action of $\mathrm{GL}(V)^{G}$ on $H^{0}\left(G \cdot x_{0}, \mathcal{E}\right)^{G}$ described in [PVS12, p. 1777] and recalled at the start of Section 2 above.

Set $D=E \backslash\{\lambda\}$ and recall the map $\sigma_{D}: \mathbb{C}^{\times} \rightarrow \mathrm{GL}(V)^{G}$ of [PVS12. p. 1784]: for $t \in \mathbb{C}^{\times}$, the element $\sigma_{D}(t)$ of $\mathrm{GL}(V)^{G}$ is defined by $\sigma_{D}(t) \cdot\left(w_{1}+w_{2}\right)=t w_{1}+w_{2}$, for all $w_{1} \in V_{1}$ and $w_{2} \in V_{2}$.

We now argue as in the proof of Part i) of [PVS12, Proposition 3.4]. The homomorphism $f: T \rightarrow \mathrm{GL}(V)^{G}$ of (2.3) is surjective (because $E$ is linearly independent), and therefore the homomorphism $f^{*}: X\left(\mathrm{GL}(V)^{G}\right) \rightarrow X(T), \delta \mapsto \delta \circ f$ of character groups is injective. Then $\delta:=\left(f^{*}\right)^{-1}(\beta)$ is the $\mathrm{GL}(V)^{G}$-weight of $s$. Consequently

$$
\begin{aligned}
s\left(z_{t}\right)=s\left(\sigma_{D}(t) \cdot x_{0}\right) & =\delta\left(\sigma_{D}(t)\right)^{-1} \sigma_{D}(t) \cdot s\left(x_{0}\right) \\
& =\delta\left(\sigma_{D}(t)\right)^{-1}\left[\sigma_{D}(t) \cdot v\right] \in V / \mathfrak{g} \cdot z_{t} .
\end{aligned}
$$

Since, by definition, $a$ is the coefficient of $\lambda$ in the expression of $\beta$ as a $\mathbb{Z}$-linear combination of the elements of $E$, we have that $\delta\left(\sigma_{D}(t)\right)=t^{a}$. Consequently

$$
s\left(z_{t}\right)=t^{-a}\left[\sigma_{D}(t) \cdot v\right] .
$$

Taking into account that

$$
\sigma_{D}(t) \cdot v=\sum_{i=2}^{m} b_{i} y_{i}+t \sum_{i=m+1}^{n} b_{i} y_{i}
$$

the proposition follows. 
We prove part $\mathrm{A}$ ) of Theorem 2.8. Assume $a \leq 0$. Since the $s_{y_{i}}$ are sections defined over the whole $X_{0}$, Proposition 2.18 implies that $s^{*}$ extends to a section over $C_{0}$. Hence $s$ extends to $Z$ by Proposition 2.16 .

For the rest of the proof we separate into five cases. Recall that $x_{0}=\sum_{\lambda \in E} v_{\lambda}$.

Case 1: $\beta$ is a root, $X_{-\beta} \cdot v_{\lambda} \neq 0$ and there exists $\mu \in E \backslash\{\lambda\}$ with $X_{-\beta} \cdot v_{\mu} \neq 0$.

Case 2: $\beta$ is a root, $X_{-\beta} \cdot v_{\lambda}=0$ and there exists $\mu \in E \backslash\{\lambda\}$ with $X_{-\beta} \cdot v_{\mu} \neq 0$.

Case 3: $\beta$ is a root, $X_{-\beta} \cdot v_{\lambda} \neq 0$ and $X_{-\beta} \cdot v_{\mu}=0$ for all $\mu \in E \backslash\{\lambda\}$.

Case 4: $\beta$ is a root, $X_{-\beta} \cdot v_{\lambda}=0$ and $X_{-\beta} \cdot v_{\mu}=0$ for all $\mu \in E \backslash\{\lambda\}$.

Case 5: $\beta$ is not a root.

We first show that Case 3 and Case 4 cannot happen. Case 3 cannot occur, because it contradicts the assumption that $\lambda$ has codimension 1, by Proposition 2.3, Case 4 cannot occur, because $\beta \in\langle E\rangle_{\mathbb{Z}}$ by Proposition 2.6. Indeed, if $\beta$ is a root in $\langle E\rangle_{\mathbb{Z}}$, then $\{\mu \in$ $\left.E:\left\langle\beta^{\vee}, \mu\right\rangle \neq 0\right\}$ is nonempty and so $X_{-\beta} \cdot x_{0} \neq 0$.

We now prove $B$ ) and C) of Theorem 2.8 in Case 1 . We begin by choosing an appropriate basis of $V^{\beta}$. Put $y_{n}:=X_{-\beta} \cdot v_{\lambda}$, and let $y_{2}, y_{3}, \ldots, y_{q}$ be the elements of

$$
\left\{X_{-\beta} \cdot v_{\mu}: \mu \in E \backslash\{\lambda\},\left\langle\beta^{\vee}, \mu\right\rangle \neq 0\right\}
$$

in some order. Finally, extend $y_{2}, y_{3}, \ldots, y_{q}, y_{n}$ to a basis $y_{2}, y_{3}, \ldots, y_{n}$ of $V^{\beta}$ such that $y_{i} \in V_{2}$ when $q+1 \leq i \leq m$ and $y_{i} \in V_{1}$ when $m+1 \leq i \leq n-1$.

Assume $t \in \mathbb{C}$. Since $\mathfrak{g} \cdot z_{t} \subset T_{z_{t}} X_{0}$, we have $X_{-\beta} \cdot z_{t} \in T_{z_{t}} X_{0}$. Hence there is, in $V / T_{z_{t}} X_{0}$, the following equality

$$
\left[y_{2}\right]=-\sum_{i=3}^{q}\left[y_{i}\right]-t\left[y_{n}\right] .
$$

Using Lemma2.17 it follows that the classes, in $V / T_{z_{t}} X_{0}$, of $y_{3}, \ldots, y_{n}$ are a basis for the image of $V^{\beta}$ in $V / T_{z_{t}} X_{0}$. In other words, the elements $s_{y_{i}}\left(z_{t}\right)$, for $3 \leq i \leq n$, are linearly independent for every $t \in \mathbb{C}$.

Combining the relation (2.12) with Proposition 2.18 we get for all nonzero $t$ that

$$
\begin{aligned}
s\left(z_{t}\right)=t^{-a}\left(\sum_{i=3}^{q}\left(b_{i}-b_{2}\right) s_{y_{i}}\left(z_{t}\right)\right)+t^{-a}\left(\sum_{i=q+1}^{m} b_{i} s_{y_{i}}\left(z_{t}\right)\right)+ \\
t^{-a+1}\left(\sum_{i=m+1}^{n-1} b_{i} s_{y_{i}}\left(z_{t}\right)\right)+t^{-a+1}\left(b_{n}-b_{2}\right) s_{y_{n}}\left(z_{t}\right) .
\end{aligned}
$$

We now prove part B) in Case 1. Assume $a>1$. We assume that $s$ extends and we will get a contradiction. Since $s$ extends we have that $s^{*}$ also extends. Since the set $\left\{s_{y_{i}}\left(z_{t}\right): 3 \leq i \leq n\right\}$ is linearly independent for all $t \in \mathbb{C}$, and $-a+1$ and $-a$ are negative, Equation (2.13) implies that $b_{i}=b_{2}$ for $3 \leq i \leq q$ and for $i=n$, and that $b_{i}=0$ for $q+1 \leq i \leq n-1$. Hence $v=b_{2}\left(X_{-\beta} \cdot x_{0}\right)$, contradicting the assumption $v \notin \mathfrak{g} \cdot x_{0}$. This proves part B) in Case 1 .

We now prove part (C) in Case 1. Assume that $a=1$. Since $-a+1=0$ and $-a<0$, arguing similarly to the case $a>1$ we get that $s^{*}$ extends over $C_{0}$ if and only if $b_{i}=b_{2}$ for 
$3 \leq i \leq q$ and $b_{i}=0$ for $q+1 \leq i \leq m$. If these conditions hold then $v-\hat{v} \in \mathfrak{g} \cdot x_{0}$, where

$$
\hat{v}=\sum_{i=m+1}^{n-1} b_{i} y_{i}+\left(b_{n}-b_{2}\right) y_{n},
$$

which is an element of $V_{1}$. Conversely, assume there exists $\hat{v} \in V_{1}$ with $v-\hat{v} \in \mathfrak{g} \cdot x_{0}$. Then $v$ and $\hat{v}$ define the same equivariant section $s$ of $\mathcal{E}$ over $G \cdot x_{0}$. Hence we can assume in Equation (2.11) that $b_{i}=0$ for $2 \leq i \leq m$. As a consequence, Proposition 2.18 implies that $s^{*}$ extends, hence by Proposition $2.16 s$ also extends. This finishes the proof of part C) of Theorem 2.8 in Case 1.

The arguments for the proof of $\mathrm{B}$ ) and (C) of Theorem 2.8 for Cases 2 and 5 are very similar to those of Case 1 so we only sketch them.

In Case 2, we can choose a basis $y_{2}, y_{3}, \ldots, y_{n}$ of $V^{\beta}$ with the following properties:

- $\left\{y_{2}, y_{3}, \ldots y_{q}\right\}=\left\{X_{-\beta} \cdot v_{\mu}: \mu \in E,\left\langle\beta^{\vee}, \mu\right\rangle \neq 0\right\}$;

- $y_{i} \in V_{2}$ for $q+1 \leq i \leq m ;$ and

- $y_{i} \in V_{1}$ for $m+1 \leq i \leq n$.

Then, for all $t \in \mathbb{C}$ we have the following equality in $V / T_{z_{t}}\left(X_{0}\right)$ :

$$
\left[y_{2}\right]=-\sum_{i=3}^{q}\left[y_{i}\right]
$$

and, similarly to Case 1 , the elements $s_{y_{i}}\left(z_{t}\right)$, for $3 \leq i \leq n$, are linearly independent. Continuing as in the proof of Case 1 the result follows.

In Case 5 , we choose a basis $y_{2}, \ldots, y_{n}$ of $V^{\beta}$ with the following properties:

- $y_{i} \in V_{2}$ for $2 \leq i \leq m$; and

- $y_{i} \in V_{1}$ for $m+1 \leq i \leq n$.

Then for all $t \in \mathbb{C}$ the elements $s_{y_{i}}\left(z_{t}\right)$, for $2 \leq i \leq n$, are linearly independent. Continuing as in the proof of Case 1 the result follows. This finishes the proof of Theorem 2.8 .

2.4. A few more facts about $T_{X_{0}} \mathrm{M}_{\mathcal{S}}$. In this subsection we prove three more facts about $T_{\text {ad }}$-weights in $T_{X_{0}} \mathrm{M}_{\mathcal{S}}$. Let $\beta$ be such a weight, and let $\beta=\sum_{\lambda \in E} a_{\lambda} \lambda$ be the unique expression of $\beta$ as a $\mathbb{Z}$-linear combination of the elements of $E$. Proposition 2.19 guarantees that at least one of the $a_{\lambda}$ is positive. Proposition 2.20, which is a consequence of a classical result attributed to Kostant, bounds the number and size of positive coefficients $a_{\lambda}$. Finally, Proposition 2.21 gives a sufficient condition for $\beta$ to be a simple root and describes the $T_{\text {ad }}$-weight space of weight $\beta$ when the condition is met. The first two propositions do not use our extension criterion (Theorem 2.8), while the third one does.

Proposition 2.19. Let $\beta$ be a $T_{\text {ad }}$-weight in $\left(V / \mathfrak{g} \cdot x_{0}\right)^{G_{x_{0}}}$. Then there exists a simple root $\alpha$ such that $\left\langle\alpha^{\vee}, \beta\right\rangle>0$. Consequently, if $\beta=\sum_{\lambda \in E} a_{\lambda} \lambda$ is the unique expression of $\beta$ as a $\mathbb{Z}$-linear combination of the elements of $E$, then there exists $\lambda \in E$ with $\left\langle\alpha^{\vee}, \lambda\right\rangle>0$ and $a_{\lambda}>0$.

Proof. This follows by a standard argument from the fact, recalled in Proposition 2.6, that $\beta$ is a nonzero element of $\langle\Pi\rangle_{\mathbb{N}}$. For completeness, we include the details. Recall that we can equip the vector space $\Lambda_{R} \otimes_{\mathbb{Z}} \mathbb{R}$, where $\Lambda_{R}=\langle\Pi\rangle_{\mathbb{Z}}$ is the root lattice, with a positive definite inner product $(\cdot \mid \cdot)$ such that for all $\alpha \in \Pi$ and all $\gamma \in \Lambda_{R}$, we have that $\left\langle\alpha^{\vee}, \gamma\right\rangle$ is a positive multiple of $(\alpha \mid \gamma)$, see e.g. [TY05, $\S 18.3$ and $\S 18.4]$. 
Since $\beta$ is an element of $\langle\Pi\rangle_{\mathbb{N}}$ there exists, for every $\alpha \in \Pi$, a nonnegative integer $n_{\alpha}$ such that $\beta=\sum_{\alpha \in \Pi} n_{\alpha} \alpha$. Since $\beta \neq 0$, we know by the positive definiteness and the bilinearity of $(\cdot \mid \cdot)$ that

$$
0<(\beta \mid \beta)=\sum_{\alpha \in \Pi} n_{\alpha}(\alpha \mid \beta)
$$

It follows that there is some $\alpha \in \Pi$ for which $(\alpha \mid \beta)>0$, whence $\left\langle\alpha^{\vee}, \beta\right\rangle>0$, which is what we had to prove.

Proposition 2.20. Let $\beta$ be a $T_{\mathrm{ad}}$-weight in $T_{X_{0}} \mathrm{M}_{\mathcal{S}}$. If $\beta=\sum_{\lambda \in E} a_{\lambda} \lambda$ is the unique expression of $\beta$ as a $\mathbb{Z}$-linear combination of the elements of $E$, then the sum of the elements of the set

$$
\left\{a_{\lambda}: \lambda \in E \text { and } a_{\lambda}>0\right\}
$$

is at most 2.

Proof. This is a consequence of the following fact, attributed to Kostant (see, e.g., [Tim11, Proposition 28.6]): the ideal $I=I\left(X_{0}\right)$ of the subvariety $X_{0}$ of $V$ is generated by the intersection, which we denote by $I_{2}$, of $I$ with the subspace $\mathbb{C}[V]_{2}$ of polynomials of degree 2 in $\mathbb{C}[V]$. If we number the elements of $E$ as $\left\{\lambda_{1}, \lambda_{2}, \ldots, \lambda_{p}\right\}$, then

$$
\mathbb{C}[V]_{2} \cong\left[\oplus_{i=1}^{p} S^{2} V\left(\lambda_{i}\right)\right] \oplus\left[\oplus_{1 \leq i<j \leq p} V\left(\lambda_{i}\right) \otimes V\left(\lambda_{j}\right)\right]
$$

as $G$-modules.

It is shown in [AB05] (and reviewed in [PVS12, Section 2.1]) that because $E$ is linearly independent $\mathrm{M}_{\mathcal{S}}$ can be identified with an open subscheme of the invariant Hilbert scheme $\operatorname{Hilb}_{\mathcal{S}}^{G}(V)$. It therefore follows from [AB05, Proposition 1.13] (and its proof) that we have natural isomorphisms

$$
T_{X_{0}} \mathrm{M}_{\mathcal{S}} \cong \operatorname{Hom}_{\mathbb{C}\left[X_{0}\right]}^{G}\left(I / I^{2}, \mathbb{C}\left[X_{0}\right]\right) \cong \operatorname{Hom}_{\mathbb{C}[V]}^{G}(I, \mathbb{C}[V] / I) .
$$

Recall from [PVS12, Section 2.2] that, as reviewed at the start of Section 2 above, the $T_{\text {ad }}$-action on $T_{X_{0}} \mathrm{M}_{\mathcal{S}} \cong \operatorname{Hom}_{\mathbb{C}[V]}^{G}(I, \mathbb{C}[V] / I)$ is induced by the action of $\mathrm{GL}(V)^{G}$ on $V$, using the homomorphism $f: T \rightarrow \mathrm{GL}(V)^{G}$ of (2.3) on page 5. The $\mathrm{GL}(V)^{G}$-action on $\operatorname{Hom}_{\mathbb{C}[V]}^{G}(I, \mathbb{C}[V] / I)$ induced by the $\mathrm{GL}(V)^{G}$-action on $V$ is given by

$$
(t \cdot \rho)(h)=t \cdot \rho\left(t^{-1} \cdot h\right)
$$

for $\rho \in \operatorname{Hom}_{\mathbb{C}[V]}^{G}(I, \mathbb{C}[V] / I), t \in \mathrm{GL}(V)^{G}$ and $h \in I$. Clearly, $\rho$ is completely determined by its restriction to $I_{2}$, since $I_{2}$ generates $I$ as an ideal. Being $G$-equivariant, $\rho$ sends each irreducible $G$-submodule $M$ of $I_{2}$ to 0 or to a $G$-submodule of $\mathbb{C}[V] / I$ isomorphic to $M$.

Since $\mathbb{C}[V] / I=\mathbb{C}\left[X_{0}\right]$, the $G$-module structure of this algebra is given by

$$
\mathbb{C}[V] / I \cong \oplus_{\mu \in \mathcal{S}} V(\mu)=\oplus_{\left(b_{i}\right) \in \mathbb{N}^{p}} V\left(\sum_{i=1}^{p} b_{i} \lambda_{i}^{*}\right) .
$$

Moreover, as stated in the first paragraph of the proof of [Tim11, Proposition 28.6], $V\left(\sum_{i} b_{i} \lambda_{i}^{*}\right) \subseteq$ $\mathbb{C}[V] / I$ is the image of $S^{b_{1}} V\left(\lambda_{1}^{*}\right) \otimes S^{b_{2}} V\left(\lambda_{2}^{*}\right) \otimes \ldots \otimes S^{b_{p}} V\left(\lambda_{p}^{*}\right) \subseteq \mathbb{C}[V]$ under the quotient map $\mathbb{C}[V] \rightarrow C[V] / I$. It follows that $t=\left(t_{1}, t_{2}, \ldots, t_{p}\right) \in \mathrm{GL}(V)^{G}$ acts on $x \in$ 
$V\left(\sum_{i} b_{i} \lambda_{i}^{*}\right) \subseteq \mathbb{C}[V] / I$ by

$$
t \cdot x=t_{1}^{-b_{1}} t_{2}^{-b_{2}} \ldots t_{p}^{-b_{p}} x,
$$

since $t \mapsto t_{1}^{-b_{1}} t_{2}^{-b_{2}} \ldots t_{p}^{-b_{p}}$ is the character by which $\mathrm{GL}(V)^{G}$ acts on $S^{b_{1}} V\left(\lambda_{1}^{*}\right) \otimes S^{b_{2}} V\left(\lambda_{2}^{*}\right) \otimes$ $\cdots \otimes S^{b_{p}} V\left(\lambda_{p}^{*}\right)$.

Now, suppose that $\rho$ is a $T_{\text {ad }}$-eigenvector in $\operatorname{Hom}_{\mathbb{C}[V]}^{G}(I, \mathbb{C}[V] / I)$ of weight $\beta$, and let $\beta=\sum_{i=1}^{p} a_{i} \lambda_{i}$ be the expression of $\beta$ as a $\mathbb{Z}$-linear combination of the elements of $E$. The homomorphism $f: T \rightarrow \mathrm{GL}(V)^{G}$ in (2.3) on page 5 relates the $T_{\text {ad }}$-action to the action of $\mathrm{GL}(V)^{G}$. Since $\beta \in\langle E\rangle_{\mathbb{Z}}$ and $E$ is linearly independent there exists a unique character $\delta$ of $\mathrm{GL}(V)^{G}$ such that $\delta \circ f=\beta$. If $t=\left(t_{1}, \ldots, t_{p}\right) \in \mathrm{GL}(V)^{G}$, then $\delta(t)=t_{1}^{a_{1}} t_{2}^{a_{2}} \ldots t_{p}^{a_{p}}$. Moreover, $\delta$ is the $\operatorname{GL}(V)^{G}$-weight of $\rho$, which means that for $t=\left(t_{1}, t_{2}, \ldots, t_{p}\right) \in \mathrm{GL}(V)^{G}$, we have

$$
t \cdot \rho=t_{1}^{a_{1}} t_{2}^{a_{2}} \ldots t_{p}^{a_{p}} \rho .
$$

Since $\rho \neq 0$ there exists an irreducible submodule $M$ of $I_{2}$ and an element $h$ of $M$ such that $\rho(h) \neq 0$. Because $I_{2} \subseteq \mathbb{C}[V]_{2}$, it follows from the decomposition (2.15) that there exist $i, j \in\{1,2, \ldots p\}$, not necessarily distinct, such that for $t=\left(t_{1}, t_{2}, \ldots, t_{p}\right) \in \mathrm{GL}(V)^{G}$ we have

$$
t \cdot h=t_{i}^{-1} t_{j}^{-1} h,
$$

since this is the action of $\operatorname{GL}(V)^{G}$ on $\mathbb{C}[V]_{2}$. Since $\rho(M) \neq 0$, there exists $\left(b_{i}\right) \in \mathbb{N}^{p}$ such that $\rho(M)=V\left(\sum_{i=1}^{p} b_{i} \lambda_{i}^{*}\right) \subseteq \mathbb{C}[V] / I$.

We then have for all $t=\left(t_{1}, \ldots, t_{p}\right) \in \mathrm{GL}(V)^{G}$ that

$$
\begin{aligned}
(t \cdot \rho)(h) & =t \cdot \rho\left(t^{-1} \cdot h\right) \\
& =t_{1}^{-b_{1}} t_{2}^{-b_{2}} \ldots t_{p}^{-b_{p}}\left(\rho\left(t_{i} t_{j} h\right)\right) \\
& =t_{i} t_{j} t_{1}^{-b_{1}} t_{2}^{-b_{2}} \ldots t_{p}^{-b_{p}}(\rho(h))
\end{aligned}
$$

where the second equality uses equations (2.17) and (2.19) and the third equality uses the $\mathbb{C}$-linearity of $\rho$. The proposition now follows from comparing (2.22) with equation (2.18).

Proposition 2.21. Let $\beta$ be a $T_{\text {ad }}$-weight in $T_{X_{0}} \mathrm{M}_{\mathcal{S}}$. If there exists $\lambda \in E$ satisfying the following two properties:

- $\lambda$ is of codimension one;

- $\lambda$ has a positive coefficient in the unique expression of $\beta$ as a linear combination of the elements of $E$;

then $\beta$ is a simple root. Moreover $\left\langle\beta^{\vee}, \lambda\right\rangle \neq 0$ and the $T_{\mathrm{ad}}$-weight space in $H^{0}\left(X_{0}, \mathcal{N}_{X_{0} \mid V}\right)^{G} \simeq$ $T_{X_{0}} \mathrm{M}_{\mathcal{S}}^{G}$ of weight $\beta$ is spanned by the section induced by $\left[X_{-\beta} v_{\lambda}\right] \in\left(V / \mathfrak{g} \cdot x_{0}\right)^{G_{x_{0}}}$.

Proof. Since $\beta$ is a $T_{\text {ad }}$-weight in $T_{X_{0}} \mathrm{M}_{\mathcal{S}} \simeq H^{0}\left(X_{0}, \mathcal{N}_{X_{0} \mid V}\right)^{G}$, there exists $v \in V$ of $T_{\text {ad }^{-}}$ weight $\beta$ such that $[v]$ is a nonzero element of $\left(V / \mathfrak{g} \cdot x_{0}\right)^{G_{x_{0}}}$ and such that the corresponding section in $H^{0}\left(G \cdot x_{0}, \mathcal{N}_{X_{0} \mid V}\right)^{G}$ extends to $Z=G \cdot x_{0} \cup G \cdot\left(x_{0}-v_{\lambda}\right)$. By Theorem 2.8(C), 
we may assume that $v \in V(\lambda) \subseteq V$. To get a contradiction, we assume that $\beta$ is not a simple root. Then, by Proposition 2.6, there exists a simple root $\alpha$ such that $\beta-\alpha$ is a positive root and

$$
0 \neq X_{\alpha} \cdot v \in\left\langle X_{-(\beta-\alpha)} x_{0}\right\rangle_{C} .
$$

Because $v \in V(\lambda)$, we have that

$$
X_{\alpha} \cdot v \in V(\lambda) .
$$

On the other hand, because $\lambda$ is of codimension one, there exists $\lambda^{\prime} \in E \backslash\{\lambda\}$ such that $\left\langle(\beta-\alpha)^{\vee}, \lambda^{\prime}\right\rangle \neq 0$. Consequently, the line $\left\langle X_{-(\beta-\alpha)} x_{0}\right\rangle_{\mathbb{C}}$ has nonzero projection on $V\left(\lambda^{\prime}\right)$. We have shown that (2.23) and (2.24) are in contradiction. That is, we have shown that $\beta$ is a simple root.

By elementary highest weight theory, the $T_{\text {ad }}$-weight space of weight $\beta$ in $V(\lambda)$ is $\left\langle X_{-\beta} v_{\lambda}\right\rangle_{C}$. This implies the second assertion.

\section{Proof of Theorem 1.2}

In this section, we prove Theorem 1.2 through case-by-case verification: we verify that the theorem holds for each saturated indecomposable spherical module in List 3.1 below. The definition of 'saturated' and 'indecomposable' can be found in [Kno98, Section 5] or in [PVS12, Definition 4.1]. The eight families (K1), (K2), (K3), (K15), (K16), (K17), (K18) and (K21) were the subject of [PVS12, Section 5]. Each subsection of this section corresponds to one of the remaining families in the list: the proposition in each subsection asserts that Theorem 1.2 holds for the family under consideration.

List 3.1 (Knop's List [Kno98, Section 5]). The saturated indecomposable spherical modules $(\bar{G}, W)$ are

(K1) $\left(\mathrm{GL}(m) \times \mathrm{GL}(n), \mathbb{C}^{m} \otimes \mathbb{C}^{n}\right)$ with $1 \leq m \leq n$;

(K2) $\left(\mathrm{GL}(n), \mathrm{Sym}^{2} \mathbb{C}^{n}\right)$ with $1 \leq n$;

(K3) $\left(\mathrm{GL}(n), \wedge^{2} \mathbb{C}^{n}\right)$ with $2 \leq n$;

(K4) $\left(\operatorname{Sp}(2 n) \times \mathbb{C}^{\times}, \mathbb{C}^{2 n}\right)$ with $1 \leq n$;

(K5) $\left(\mathrm{Sp}(2 n) \times \mathrm{GL}(2), \mathbb{C}^{2 n} \otimes \mathbb{C}^{2}\right)$ with $2 \leq n$;

(K6) $\left(\mathrm{Sp}(2 n) \times \mathrm{GL}(3), \mathrm{C}^{2 n} \otimes \mathbb{C}^{3}\right)$ with $3 \leq n$;

(K7) $\left(\mathrm{Sp}(4) \times \mathrm{GL}(3), \mathbb{C}^{4} \otimes \mathbb{C}^{3}\right)$;

(K8) $\left(\mathrm{Sp}(4) \times \mathrm{GL}(n), \mathbb{C}^{4} \otimes \mathbb{C}^{n}\right)$ with $4 \leq n$;

(K9) $\left(\mathrm{SO}(n) \times \mathbb{C}^{\times}, \mathbb{C}^{n}\right)$ with $3 \leq n$;

(K10) $\left(\operatorname{Spin}(10) \times \mathbb{C}^{\times}, \mathbb{C}^{16}\right)$;

(K11) $\left(\operatorname{Spin}(7) \times \mathbb{C}^{\times}, \mathbb{C}^{8}\right)$;

(K12) $\left(\operatorname{Spin}(9) \times \mathbb{C}^{\times}, \mathbb{C}^{16}\right)$;

(K13) $\left(\mathrm{G}_{2} \times \mathbb{C}^{\times}, \mathbb{C}^{7}\right)$;

(K14) $\left(\mathrm{E}_{6} \times \mathbb{C}^{\times}, \mathbb{C}^{27}\right)$;

(K15) $\left(\mathrm{GL}(n) \times \mathbb{C}^{\times}, \wedge^{2} \mathbb{C}^{n} \oplus \mathbb{C}^{n}\right)$ with $4 \leq n$;

(K16) $\left(\mathrm{GL}(n) \times \mathbb{C}^{\times}, \wedge^{2} \mathbb{C}^{n} \oplus\left(\mathbb{C}^{n}\right)^{*}\right)$ with $4 \leq n$;

(K17) $\left(\mathrm{GL}(m) \times \mathrm{GL}(n),\left(\mathbb{C}^{m} \otimes \mathbb{C}^{n}\right) \oplus \mathbb{C}^{n}\right)$ with $1 \leq m, 2 \leq n$;

(K18) $\left(\mathrm{GL}(m) \times \mathrm{GL}(n),\left(\mathbb{C}^{m} \otimes \mathbb{C}^{n}\right) \oplus\left(\mathbb{C}^{n}\right)^{*}\right)$ with $1 \leq m, 2 \leq n$;

(K19) $\left(\operatorname{Sp}(2 n) \times \mathbb{C}^{\times} \times \mathbb{C}^{\times}, \mathbb{C}^{2 n} \oplus \mathbb{C}^{2 n}\right)$ with $2 \leq n$; 
(K20) $\left(\left(\mathrm{Sp}(2 n) \times \mathbb{C}^{\times}\right) \times \mathrm{GL}(2),\left(\mathbb{C}^{2 n} \otimes \mathbb{C}^{2}\right) \oplus \mathbb{C}^{2}\right)$ with $2 \leq n$;

$(\mathrm{K} 21)\left(\mathrm{GL}(m) \times \mathrm{SL}(2) \times \mathrm{GL}(n),\left(\mathbb{C}^{m} \otimes \mathbb{C}^{2}\right) \oplus\left(\mathbb{C}^{2} \otimes \mathbb{C}^{n}\right)\right)$ with $2 \leq m \leq n$;

$(\mathrm{K} 22)\left(\left(\mathrm{Sp}(2 m) \times \mathbb{C}^{\times}\right) \times \mathrm{SL}(2) \times \mathrm{GL}(n),\left(\mathbb{C}^{2 m} \otimes \mathbb{C}^{2}\right) \oplus\left(\mathbb{C}^{2} \otimes \mathbb{C}^{n}\right)\right)$ with $2 \leq m, n$;

$(\mathrm{K} 23)\left(\left(\mathrm{Sp}(2 m) \times \mathbb{C}^{\times}\right) \times \mathrm{SL}(2) \times\left(\mathrm{Sp}(2 n) \times \mathbb{C}^{\times}\right),\left(\mathbb{C}^{2 m} \otimes \mathbb{C}^{2}\right) \oplus\left(\mathbb{C}^{2} \otimes \mathbb{C}^{2 n}\right)\right)$ with $2 \leq$ $m, n$;

(K24) $\left(\operatorname{Spin}(8) \times \mathbb{C}^{\times} \times \mathbb{C}^{\times}, \mathbb{C}_{+}^{8} \oplus \mathbb{C}_{-}^{8}\right)$.

Remark 3.2. The indices $m$ and $n$ in family (K17) and family (K18) run through a larger set than that given in Knop's List in [Kno98]. Knop communicated the revised range of indices for these families to the second author. We remark that these cases do appear in the lists of [Lea98] and [BR96]. In the family (K9) we suppose that $n \geq 3$, whereas in [Kno98] it is required that $n \geq 2$. This correction to (K9) was already made in the revised version of [Kno98] available on Knop's website.

In the rest of the present section, we will use the same notations as in [PVS12, Section 5]: in each subsection, $(\bar{G}, W)$ will denote a member of the family from List 3.1 under consideration. Given such a spherical module $(\bar{G}, W)$,

- $E$ denotes the basis of the weight monoid of $\left(\bar{G}, W^{*}\right)$ (the elements of $E$ are called the 'basic weights' in [Kno98]);

- $V=\oplus_{\lambda \in E} V(\lambda)$

- $x_{0}=\sum_{\lambda \in E} v_{\lambda}$.

Except if stated otherwise, $G$ will denote a connected subgroup of $\bar{G}$ containing $\bar{G}^{\prime}$ such that $(G, W)$ is spherical. Recall that such a group $G$ is necessarily reductive. To lighten notation, we will use $G^{\prime}$ for the derived subgroup $\bar{G}^{\prime}$ of $\bar{G}$. This should not cause confusion since $(\bar{G}, \bar{G})=(G, G)=G^{\prime}$. We will use $p$ for the projection from the weight lattice of $G$ to the weight lattice of $G^{\prime}$ (where we fix the maximal torus $T \cap G^{\prime}$ of $G^{\prime}$ ). We will use $\omega, \omega^{\prime}, \omega^{\prime \prime}$ for weights of the first, second and third non-abelian factor of $G$, while $\varepsilon$ will refer to the character $\mathbb{C}^{\times} \rightarrow \mathbb{C}^{\times}, z \mapsto z$ of $\mathbb{C}^{\times}$.

Remark 3.3. Let $(\bar{G}, W)$ be a spherical module in Knop's List and let $G$ be a connected subgroup of $\bar{G}$ containing $\bar{G}^{\prime}$. Theorem 5.1 in [Kno98] gives a criterion which characterizes, in terms of the center of $G$, whether $(G, W)$ is a spherical module: $(G, W)$ is spherical if and only if the center of $G$ separates the weights in a certain subspace $\mathfrak{a}^{*} \cap \mathfrak{z}^{*}$ of the dual of the Lie algebra of the maximal torus of $\bar{G}$. The tables in [Kno98] give an explicit basis of $\mathfrak{a}^{*} \cap \mathfrak{z}^{*}$ for every spherical module $(\bar{G}, W)$ in Knop's List.

Remark 3.4. (a) We recall from [PVS12, Remark 5.4] that the $T_{\text {ad }}$-weight set we obtain below for each $T_{X_{0}} \mathbf{M}_{\mathcal{S}}^{G}$ is a basis of the monoid $-w_{0} \Sigma_{W}$, where $w_{0}$ is the longest element in the Weyl group of $G$ (instead of $-\Sigma_{W}$ as in Theorem 1.1 where the $T_{\text {ad }}$-action from [AB05] was used).

(b) As explained in [PVS12, Remark 5.6], the computations of the $T_{\text {ad }}$-weight sets of $T_{X_{0}} M_{\mathcal{S}}^{G}$ we perform in this section confirm Knop's computation in [Kno98, Section 5] of the "simple reflections" of the little Weyl group of the spherical modules under consideration. 
3.1. (K4) The modules $\left(\operatorname{Sp}(2 n) \times \mathbb{C}^{\times}, \mathbb{C}^{2 n}\right)$ with $1 \leq n$. Here

$$
\begin{aligned}
& E=\left\{\omega_{1}+\varepsilon\right\} ; \\
& d_{W}=0 .
\end{aligned}
$$

We will make use of the following general lemma to treat this case, as well as the cases (K9), (K11) and (K13) below.

Lemma 3.5. Let $G$ be a connected reductive group and let $W$ be a spherical $G$-module. If $E^{*}$ is the basis of the weight monoid $\mathcal{S}(W)$ of $W$ and $x_{0}=\sum_{\lambda \in E} v_{\lambda} \in \oplus_{\lambda \in E} V(\lambda)$, then $\operatorname{dim} W=$ $\operatorname{dim} \mathfrak{g} \cdot x_{0}$.

Proof. This follows from [JR09, Proposition 1.1] using the fact that $X_{0}=\overline{G \cdot x_{0}}$ and $W$ have the same weight monoid.

Applying this lemma to the modules $W$ in the family (K4) yields the following proposition.

Proposition 3.6. The vector space $V / \mathfrak{g} \cdot x_{0}$ is zero-dimensional. In particular, $\operatorname{dim}(V / \mathfrak{g}$. $\left.x_{0}\right)^{G_{x_{0}}^{\prime}}=d_{W}$. Consequently, $\operatorname{dim} T_{X_{0}} \mathrm{M}_{\mathcal{S}}^{G}=d_{W}$.

Proof. Since $V \simeq W$ and, by Lemma 3.5, $\operatorname{dim} \mathfrak{g} \cdot x_{0}=\operatorname{dim} W$, we have that $V / \mathfrak{g} \cdot x_{0}=$ $\{0\}$.

3.2. (K5) The modules $\left(\mathrm{Sp}(2 n) \times \mathrm{GL}(2), \mathbb{C}^{2 n} \otimes \mathbb{C}^{2}\right)$ with $2 \leq n$. Here

$$
\begin{aligned}
& E=\left\{\omega_{1}+\omega_{1}^{\prime}, \omega_{2}+\omega_{2}^{\prime}, \omega_{2}^{\prime}\right\} ; \\
& d_{W}=2 .
\end{aligned}
$$

Proposition 3.7. The $T_{\mathrm{ad}}$-module $\left(V / \mathfrak{g} \cdot x_{0}\right)^{G_{x_{0}}^{\prime}}$ is multiplicity-free and has $T_{\mathrm{ad}}$-weight set

$$
\left\{\alpha_{1}+\alpha^{\prime}, \alpha_{1}+2 \delta+\alpha_{n}\right\},
$$

where $\delta=0$ if $n=2$ and $\delta=\alpha_{2}+\alpha_{3}+\ldots+\alpha_{n-1}$ if $n>2$. In particular, $\operatorname{dim}\left(V / \mathfrak{g} \cdot x_{0}\right)^{G_{x_{0}}^{\prime}}=$ $d_{W}$. Consequently, $\operatorname{dim} T_{X_{0}} \mathrm{M}_{\mathcal{S}}^{G}=d_{W}$

Proof. Note that $G^{\prime}=\operatorname{Sp}(2 n) \times \operatorname{SL}(2)$. Consider the $G^{\prime}$-module $V^{\prime}:=V\left(\omega_{1}+\omega_{1}^{\prime}\right) \oplus$ $V\left(\omega_{2}\right)$ and its element $x_{0}^{\prime}=v_{\omega_{1}+\omega_{1}^{\prime}}+v_{\omega_{2}}$. Observe that $G_{x_{0}}^{\prime}=G_{x_{0}^{\prime}}^{\prime}$. Since $V\left(\omega_{2}^{\prime}\right)$ is onedimensional, we have that $\left(V^{\prime} / \mathfrak{g}^{\prime} \cdot x_{0}^{\prime}\right)^{G_{x_{0}}^{\prime}} \simeq\left(V / \mathfrak{g} \cdot x_{0}\right)^{G_{x_{0}}^{\prime}}$ as $T_{\text {ad }}$-modules.

Recall that $p$ is the projection from the weight lattice $\Lambda$ of $G$ to the weight lattice of $G^{\prime}$. The monoid $p\left(\langle E\rangle_{\mathbb{N}}\right)=\left\langle\omega_{1}+\omega_{1}^{\prime}, \omega_{2}\right\rangle_{\mathbb{N}}$ is free and $G^{\prime}$-saturated. By [BCF08, Theorems 3.1 and 3.10], $\left(V^{\prime} / \mathfrak{g}^{\prime} \cdot x_{0}^{\prime}\right)^{G_{x_{0}}^{\prime}}$ is multiplicity-free and its $T_{\text {ad }}$-weights belong to Table 1 in [BCF08, page 2810]. By Proposition 2.6 the $T_{\text {ad }}$-weights of $\left(V^{\prime} / \mathfrak{g}^{\prime} \cdot x_{0}^{\prime}\right)^{G_{x_{0}}^{\prime}}$ also belong to $\Lambda_{R} \cap\left\langle\omega_{1}+\omega_{1}^{\prime}, \omega_{2}\right\rangle_{\mathbb{Z}}$. A straightforward computation shows that

$$
\Lambda_{R} \cap\left\langle\omega_{1}+\omega_{1}^{\prime}, \omega_{2}\right\rangle_{\mathbb{Z}}=\left\langle\alpha_{1}+2 \delta+\alpha_{n}, \alpha_{1}+\alpha^{\prime}\right\rangle_{\mathbb{Z}}
$$

Observe that the support of each of the two generators of $\Lambda_{R} \cap\left\langle\omega_{1}+\omega_{1}^{\prime}, \omega_{2}\right\rangle_{\mathbb{Z}}$ in equation (3.1) contains a simple root not in the support of the other generator. Because the $T_{\text {ad }}$-weights of $\left(V^{\prime} / \mathfrak{g}^{\prime} \cdot x_{0}^{\prime}\right)^{G_{x_{0}}^{\prime}}$ are linear combinations of the simple roots with positive coefficents, it follows that they belong to $\left\langle\alpha_{1}+2 \delta+\alpha_{n}, \alpha_{1}+\alpha^{\prime}\right\rangle_{\mathbb{N}}$. One checks that for 
$n>2$, the only $T_{\text {ad }}$-weights in [BCF08, Table 1$]$ satisfying this requirement are $\alpha_{1}+\alpha^{\prime}$ and $\alpha_{1}+2 \delta+\alpha_{n}$.

For $n=2$ there is a third $T_{\text {ad }}$-weight that satisfies it, namely $\gamma:=2 \alpha_{1}+2 \alpha_{2}$. The weight $\gamma$ occurs in $V^{\prime}$, with multiplicity one. More precisely, this $T_{\text {ad }}$-weight space is a line $\mathbb{C} v$ in $V\left(\omega_{2}\right) \subseteq V^{\prime}$. We claim that $[v] \in \frac{V^{\prime}}{\mathfrak{g}^{\prime} \cdot x_{0}^{\prime}}$ does not belong to $\left(\frac{V^{\prime}}{\mathfrak{g}^{\prime} \cdot x_{0}^{\prime}}\right)^{G_{x_{0}}^{\prime}}$. We prove the claim by contradiction. Indeed, since $\alpha_{2}$ is the only simple root such that $\gamma-\alpha_{2} \in R^{+} \cup\{0\}$, we have that $0 \neq[v] \in\left(\frac{V^{\prime}}{\mathfrak{g}^{\prime} \cdot x_{0}^{\prime}}\right)^{G_{x_{0}}^{\prime}}$ would, by Proposition 2.6, imply that $X_{\alpha_{2}} \cdot v$ is a nonzero element of $\left\langle X_{-\left(\gamma-\alpha_{2}\right)} \cdot x_{0}^{\prime}\right\rangle_{\mathbb{C}}$. This is absurd since $X_{-\left(\gamma-\alpha_{2}\right)} \cdot x_{0}^{\prime}$ has nonzero projection onto the component $V\left(\omega_{1}+\omega_{1}^{\prime}\right)$ of $V^{\prime}$ and the claim is proved.

We have shown that $\left(V / \mathfrak{g} \cdot x_{0}\right)^{G_{x_{0}}^{\prime}}$ is multiplicity-free and that its $T_{\text {ad }}$-weight set is a subset of the one in the statement of the proposition. Since Proposition 2.5(1) of [PVS12] tells us that $\operatorname{dim} T_{X_{0}} \mathrm{M}_{\mathcal{S}}^{G} \geq d_{W}$ and Corollary 2.14 of loc.cit. says that $T_{X_{0}} \mathrm{M}_{\mathcal{S}}^{G} \subseteq(V / \mathfrak{g}$. $\left.x_{0}\right)^{G_{x_{0}}}$, the proposition follows.

3.3. (K6) The modules $\left(\mathrm{Sp}(2 n) \times \mathrm{GL}(3), \mathbb{C}^{2 n} \otimes \mathbb{C}^{3}\right)$ with $3 \leq n$. For these modules,

$$
\begin{aligned}
& E=\left\{\lambda_{1}, \lambda_{2}, \lambda_{3}, \lambda_{4}, \lambda_{5}, \lambda_{6}\right\} ; \\
& d_{W}=5,
\end{aligned}
$$

where

$$
\begin{array}{lll}
\lambda_{1}:=\omega_{1}+\omega_{1}^{\prime} ; & \lambda_{2}:=\omega_{2}+\omega_{2}^{\prime} ; & \lambda_{3}:=\omega_{3}+\omega_{3}^{\prime} ; \\
\lambda_{4}:=\omega_{2}^{\prime} ; & \lambda_{5}:=\omega_{1}+\omega_{3}^{\prime} ; & \lambda_{6}:=\omega_{2}+\omega_{1}^{\prime}+\omega_{3}^{\prime} .
\end{array}
$$

We remark that $G=\bar{G}$ is the only connected group between $\bar{G}^{\prime}$ and $\bar{G}$ for which these modules are spherical, cf. Remark 3.3. Therefore, we assume that $G=\bar{G}=\operatorname{Sp}(2 n) \times$ GL(3) throughout this section.

In this section we will prove the following proposition.

Proposition 3.8. The $T_{\mathrm{ad}}$-module $T_{X_{0}} \mathrm{M}_{\mathcal{S}}^{G}$ is multiplicity-free. Its $T_{\mathrm{ad}}$-weight set is

$$
\left\{\alpha_{1}, \alpha_{2}, \alpha_{2}+\gamma, \alpha_{1}^{\prime}, \alpha_{2}^{\prime}\right\},
$$

where $\gamma=\alpha_{3}$ if $n=3$ and $\gamma=2\left(\alpha_{3}+\alpha_{4}+\ldots+\alpha_{n-1}\right)+\alpha_{n}$ if $n>3$. In particular, $\operatorname{dim} T_{X_{0}} \mathrm{M}_{\mathcal{S}}^{G}=d_{W}$.

Proof. Let $\beta$ be a $T_{\text {ad }}$-weight in $T_{X_{0}} \mathrm{M}_{\mathcal{S}}^{G}$. It follows from Proposition 2.19 that at least one $\lambda \in E$ has a positive coefficient in the expression of $\beta$ as a $\mathbb{Z}$-linear combination of elements of $E$. Note that all elements of $E$ except $\lambda_{3}$ have codimension 1. In particular, if $\lambda \neq \lambda_{3}$, then it follows from Proposition 2.21 that $\beta$ is a simple root belonging to the set (3.2), and that its weight space has dimension one.

Since $\operatorname{dim} T_{X_{0}} \mathrm{M}_{\mathcal{S}}^{G} \geq d_{W}$ by [PVS12, Proposition 2.5(1)], what remains to prove the proposition is to show the following three claims:

Claim A: if $\lambda_{3}$ is the only element of $E$ which has a positive coefficient in the expression of $\beta$, then $\beta=\alpha_{2}+\gamma$ if $n>3$ and $\beta \in\left\{\alpha_{2}+\gamma, 2 \alpha_{2}+2 \gamma\right\}$ if $n=3$. 
Claim B: suppose $n=3$; the $T_{\text {ad }}$-weight $\beta=2 \alpha_{2}+2 \gamma$ does not occur in $(V / \mathfrak{g}$. $\left.x_{0}\right)^{G_{x_{0}}}$, and therefore also not in the subspace $T_{X_{0}} \mathrm{M}_{\mathcal{S}}^{G}$.

Claim C: the $T_{\text {ad }}$-weight $\beta=\alpha_{2}+\gamma$ has multiplicity at most one in $T_{X_{0}} M_{\mathcal{S}}^{G}$.

We begin with Claim A. Recall from Proposition 2.6 that $\beta \in\langle E\rangle_{\mathbb{Z}} \cap\langle\Pi\rangle_{\mathbb{N}}$. Straightforward computations show that

$$
\langle E\rangle_{\mathbb{Z}} \cap \Lambda_{R}=\left\langle\alpha_{1}, \alpha_{2}, \alpha_{1}^{\prime}, \alpha_{2}^{\prime}, \gamma\right\rangle_{\mathbb{Z}}
$$

and that

$$
\begin{aligned}
\alpha_{1} & =\lambda_{1}+\lambda_{5}-\lambda_{6} ; \\
\alpha_{2} & =\lambda_{2}+\lambda_{6}-\lambda_{1}-\lambda_{3}-\lambda_{4} ; \\
\alpha_{1}^{\prime} & =\lambda_{1}+\lambda_{6}-\lambda_{5}-\lambda_{2} ; \\
\alpha_{2}^{\prime} & =\lambda_{2}+\lambda_{4}-\lambda_{6} ; \\
\gamma & =2 \lambda_{3}+\lambda_{1}+\lambda_{4}-\lambda_{5}-\lambda_{2}-\lambda_{6} .
\end{aligned}
$$

Let $K$ be the basis of $\langle E\rangle_{\mathbb{Z}} \cap \Lambda_{R}$ given in equation (3.3). Since $\beta \in\langle\Pi\rangle_{\mathbb{N}}$ and all elements of $K$ contain a simple root in their support, that is not in the support of any other element of $K$, it follows that $\beta \in\langle K\rangle_{\mathbb{N}}$. Therefore, there exist $A_{1}, A_{2}, \ldots, A_{5} \in \mathbb{N}$ such that

$$
\beta=A_{1} \alpha_{1}+A_{2} \alpha_{2}+A_{3} \alpha_{1}^{\prime}+A_{4} \alpha_{2}^{\prime}+A_{5} \gamma .
$$

From the hypothesis of Claim A, it follows that

$$
\left\{\begin{array}{l}
A_{1}-A_{2}+A_{3}+A_{5} \leq 0 \\
A_{2}-A_{3}+A_{4}-A_{5} \leq 0 \\
-A_{2}+A_{4}+A_{5} \leq 0 \\
A_{1}-A_{3}-A_{5} \leq 0 \\
-A_{1}+A_{2}+A_{3}-A_{4}-A_{5} \leq 0
\end{array}\right.
$$

Adding the first two inequalities in (3.5) yields that $A_{1}=A_{4}=0$. Then adding the first and the last gives that $A_{3}=0$. After substituting these values into the first and last inequalities, we deduce that $A_{2}=A_{5}$. It follows that $\beta \in\left\langle\alpha_{2}+\gamma\right\rangle_{\mathbb{N}}$. Using that $\beta$ is the sum of a simple root and an element of $R^{+} \cup\{0\}$ (see Proposition 2.6) it follows that $\beta=\alpha_{2}+\gamma$ if $n>3$ and that $\beta \in\left\{\alpha_{2}+\gamma, 2 \alpha_{2}+2 \gamma\right\}$ if $n=3$, This proves Claim A.

We proceed to Claim B. Let $n=3$ and fix $\beta=2 \alpha_{2}+2 \gamma=2 \alpha_{2}+2 \alpha_{3}$. One deduces from the well-known decompositions into T-weight spaces of $V\left(\omega_{1}\right), V\left(\omega_{2}\right)$ and $V\left(\omega_{3}\right)$ that the $T_{\text {ad }}$-weight space in $V$ of $T_{\text {ad }}$-weight $\beta$ is a line $\mathbb{C} v$ in $V\left(\lambda_{3}\right) \subseteq V$. We prove Claim B by contradiction. Indeed, since $\alpha_{3}$ is the only simple root such that $\gamma-\alpha_{3} \in R^{+} \cup\{0\}$, we have that $0 \neq[v] \in\left(V / \mathfrak{g} \cdot x_{0}\right)^{G_{x_{0}}}$ would, by Proposition 2.6, imply that $X_{\alpha_{3}} \cdot v$ is a nonzero element of $\left\langle X_{-\left(\gamma-\alpha_{3}\right)} \cdot x_{0}\right\rangle_{C}$. This is absurd since $X_{-\left(\gamma-\alpha_{3}\right)} \cdot x_{0}$ has nonzero projection onto the components $V\left(\lambda_{2}\right)$ and $V\left(\lambda_{6}\right)$ of $V$. Claim B is proved.

Finally, we show Claim C. We fix $\beta=\alpha_{2}+\gamma$. We will show that the $T_{\text {ad }}$-weight $\beta$ has multiplicity at most one in $\left(V / \mathfrak{g} \cdot x_{0}\right)^{G_{x_{0}}}$. Since $T_{X_{0}} \mathrm{M}_{\mathcal{S}}^{G} \subseteq\left(V / \mathfrak{g} \cdot x_{0}\right)^{G_{x_{0}}}$, this implies Claim C. First off, we claim that the $T_{\mathrm{ad}}$-weight $\beta$ only occurs in $V\left(\lambda_{2}\right), V\left(\lambda_{3}\right)$ and in $V\left(\lambda_{6}\right)$. Indeed, $\beta$ belongs to the root lattice of $\operatorname{Sp}(2 n)$, and does not occur as a $T_{\text {ad }}$-weight 
in $V\left(\omega_{1}\right)$. Let $Z$ be the subspace of $V\left(\lambda_{2}\right) \oplus V\left(\lambda_{3}\right) \oplus V\left(\lambda_{6}\right)$ consisting of $T_{\text {ad }}$-eigenvectors $v$ of $T_{\text {ad }}$-weight $\beta$ that satisfy the following three conditions:

$$
\begin{aligned}
& X_{\alpha_{2}} \cdot v \in\left\langle X_{-\left(\beta-\alpha_{2}\right)} x_{0}\right\rangle_{C} ; \\
& X_{\alpha_{3}} \cdot v \in\left\langle X_{-\left(\beta-\alpha_{3}\right)} x_{0}\right\rangle_{C} ; \text { and } \\
& X_{\alpha_{k}} \cdot v=0 \text { for all } k \in\{1,2, \ldots, n\} \backslash\{2,3\} .
\end{aligned}
$$

Since $\alpha=\alpha_{2}$ and $\alpha=\alpha_{3}$ are the only simple roots such that $\beta-\alpha \in R^{+} \cup\{0\}$ it follows from Proposition 2.6 that every $v \in V^{\beta}$ such that $0 \neq[v] \in\left(V / \mathfrak{g} \cdot x_{0}\right)^{G_{x_{0}}}$ satisfies (3.6), (3.7) and (3.8). To show Claim $C$ it is therefore enough to prove that

$$
\operatorname{dim} Z \leq 2,
$$

since the nonzero vector $X_{-\beta} \cdot x_{0}$ belongs to $\mathfrak{g} \cdot x_{0} \cap Z$.

To prove the inequality (3.9) we will make use of the explicit description of $\mathfrak{s p}(2 n)$ and its root operators given in the proof of [GW09, Theorem 2.4.1], as well as the notations therein. In particular, we have a basis $\left\{e_{1}, e_{2}, \ldots, e_{n}, e_{-1}, e_{-2}, \ldots, e_{-n}\right\}$ of $\mathbb{C}^{2 n}$ and a $\mathbb{Z}$-basis $\left\{\varepsilon_{1}, \varepsilon_{2}, \ldots, \varepsilon_{n}\right\}$ of the weight lattice of $\operatorname{Sp}(2 n)$ such that $e_{i}$ has weight $\varepsilon_{i}$ and $e_{-k}$ has weight $-\varepsilon_{k}$ in the defining representation of $\operatorname{Sp}(2 n)$ on $\mathbb{C}^{2 n}$. In terms of the basis $\left\{\varepsilon_{1}, \varepsilon_{2}, \ldots, \varepsilon_{n}\right\}$ of the weight lattice, the simple roots of $\operatorname{Sp}(2 n)$ are $\alpha_{i}=\varepsilon_{i}-\varepsilon_{i+1}$ for $i \in\{1,2, \ldots, n-1\}$ and $\alpha_{n}=2 \varepsilon_{n}$. Moreover, for each root $\delta$ we have a root operator $X_{\delta} \in \mathfrak{s p}(2 n)^{\delta}$. In view of conditions (3.6), (3.7) and (3.8) we will make use of the root operators associated to the simple roots and to the negative roots $-\left(\beta-\alpha_{2}\right)=-2 \varepsilon_{3}$ and $-\left(\beta-\alpha_{3}\right)=-\varepsilon_{2}-\varepsilon_{4}$. The action of these operators on the given basis of the defining representation $\mathbb{C}^{2 n}$ of $\operatorname{Sp}(2 n)$ is as follows:

$$
\begin{aligned}
& X_{\alpha_{i}} \cdot e_{k}=\left\{\begin{array}{ll}
e_{i} & \text { if } k=i+1 ; \\
-e_{-(i+1)} & \text { if } k=-i ; \\
0 & \text { if } k \notin\{i,-i\}
\end{array} \quad \text { for } i \in\{1,2, \ldots, n-1\} ;\right. \\
& X_{\alpha_{n}} \cdot e_{k}= \begin{cases}e_{n} & \text { if } k=-n ; \\
0 & \text { if } k \neq-n ;\end{cases} \\
& X_{-2 \varepsilon_{i}} \cdot e_{k}=\left\{\begin{array}{ll}
e_{-i} & \text { if } k=i ; \\
0 & \text { if } k \neq i
\end{array} \quad \text { for } i \in\{1,2, \ldots, n\} ;\right. \\
& X_{-\varepsilon_{i}-\varepsilon_{j}} \cdot e_{k}= \begin{cases}e_{-j} & \text { if } k=i \\
e_{-i} & \text { if } k=j ; \\
0 & \text { if } k \notin\{i, j\}\end{cases}
\end{aligned}
$$

Note that

$$
\beta=\alpha_{2}+\gamma=-\omega_{1}+\omega_{3}=\varepsilon_{2}+\varepsilon_{3} .
$$

We now identify the weight spaces of this $T_{\text {ad }}$-weight in the representations $V\left(\omega_{2}\right)$ and $V\left(\omega_{3}\right)$ of $\operatorname{Sp}(2 n)$. A vector in $V\left(\omega_{2}\right)$ has $T_{\text {ad }}$-weight $\beta$ if and only if it has $T$-weight $\omega_{2}-$ $\beta=\varepsilon_{1}-\varepsilon_{3}$. We identify $V\left(\omega_{2}\right)$ with the sub-Sp $(2 n)$-representation of $\wedge^{2} \mathbb{C}^{2 n}$ with highest weight vector $e_{1} \wedge e_{2}$. Then the $T$-weight space in $V\left(\omega_{2}\right)$ of weight $\varepsilon_{1}-\varepsilon_{3}$ is the line 
spanned by

$$
e_{1} \wedge e_{-3}
$$

A vector in $V\left(\omega_{3}\right)$ has $T_{\text {ad }}$-weight $\beta$ if and only if it has $T$-weight $\omega_{3}-\beta=\varepsilon_{1}$. As is well-known, $V\left(\omega_{3}\right)$ is the irreducible component of the $\operatorname{Sp}(2 n)$-module $\wedge^{3} \mathbb{C}^{2 n}$ generated by the highest weight vector $e_{1} \wedge e_{2} \wedge e_{3}$. In the larger module $\wedge^{3} \mathbb{C}^{2 n}$ the $T$-weight space of weight $\varepsilon_{1}$ is spanned by the following vectors

$$
e_{1} \wedge e_{2} \wedge e_{-2}, e_{1} \wedge e_{3} \wedge e_{-3}, \ldots, e_{1} \wedge e_{n} \wedge e_{-n} .
$$

It follows from the previous paragraph that if $v \in Z$, then there exist $A_{1}, A_{2} \in \mathbb{C}$ and $B_{2}, B_{3}, \ldots, B_{n} \in \mathbb{C}$ such that

$$
v=A_{1}\left(e_{1} \wedge e_{-3} \otimes v_{\omega_{2}^{\prime}}\right)+A_{2}\left(e_{1} \wedge e_{-3} \otimes v_{\omega_{1}^{\prime}+\omega_{3}^{\prime}}\right)+\sum_{k=2}^{n} B_{k}\left(e_{1} \wedge e_{k} \wedge e_{-k} \otimes v_{\omega_{3}^{\prime}}\right) .
$$

Straightforward computations using the root operators show that conditions (3.6), (3.7) and (3.8) imply that

$$
\begin{aligned}
& A_{1}=A_{2}=-B_{3}+B_{4} ; \\
& B_{4}=B_{5}=\ldots=B_{n} .
\end{aligned}
$$

This implies that $\operatorname{dim} Z \leq 3$. Note that the vector $v_{1} \otimes v_{\omega_{3}^{\prime}} \in \wedge^{3} \mathbb{C}^{2 n} \otimes V\left(\omega_{3}^{\prime}\right)$, where

$$
v_{1}=\sum_{k=2}^{n} e_{1} \wedge e_{k} \wedge e_{-k}
$$

satisfies the equations (3.17) and (3.18). It is straightforward to check that $v_{1}$ is a highest weight vector. It follows that $v_{1}$ is an element of the $\operatorname{Sp}(2 n)$-stable complement to $V\left(\omega_{3}\right)$ in $\wedge^{3} \mathbb{C}^{2 n}$. Consequently, the line spanned by $v_{1} \otimes v_{\omega_{3}^{\prime}}$ is not contained in $Z$, and $\operatorname{dim} Z \leq$ 2. This proves Claim $\mathrm{C}$, and the proposition.

3.4. (K7) The module $\left(\operatorname{Sp}(4) \times \mathrm{GL}(3), \mathbb{C}^{4} \otimes \mathbb{C}^{3}\right)$. For this module we have

$$
\begin{aligned}
E & =\left\{\omega_{1}+\omega_{1}^{\prime}, \omega_{2}+\omega_{2}^{\prime}, \omega_{2}^{\prime}, \omega_{1}+\omega_{3}^{\prime}, \omega_{2}+\omega_{1}^{\prime}+\omega_{3}^{\prime}\right\} \\
d_{W} & =4 .
\end{aligned}
$$

Proposition 3.9. The $T_{\mathrm{ad}}$-module $T_{X_{0}} \mathrm{M}_{\mathcal{S}}^{G}$ is multiplicity-free. Its $T_{\mathrm{ad}}$-weight set is

$$
\left\{\alpha_{1}, \alpha_{2}, \alpha_{1}^{\prime}, \alpha_{2}^{\prime}\right\} \text {. }
$$

In particular, $\operatorname{dim} T_{X_{0}} \mathrm{M}_{\mathcal{S}}^{G}=d_{W}$.

Proof. Let $\beta$ be a $T_{\text {ad }}$-weight in $T_{X_{0}} \mathrm{M}_{\mathcal{S}}^{G}$. It follows from Proposition 2.19 that at least one $\lambda \in E$ has a positive coefficient in the expression of $\beta$ as a $\mathbb{Z}$-linear combination of elements of $E$. Note that all elements of $E$ have codimension 1. It follows from Proposition 2.21 that $\beta$ is a simple root and that its weight space has dimension one. Since the set (3.19) contains all simple roots of $G$, we can conclude that $\beta$ belongs to this set and that $\operatorname{dim} T_{X_{0}} \mathrm{M}_{\mathcal{S}}^{G} \leq d_{W}$. The proposition now follows from the a priori estimate $\operatorname{dim} T_{X_{0}} \mathrm{M}_{\mathcal{S}}^{G} \geq d_{W}$, see [PVS12, Proposition 2.5(1)]. 
3.5. (K8) The modules $\left(\mathrm{Sp}(4) \times \mathrm{GL}(n), \mathbb{C}^{4} \otimes \mathbb{C}^{n}\right)$ with $4 \leq n$. We put $\lambda_{1}=\omega_{1}+\omega_{1}^{\prime}, \lambda_{2}=$ $\omega_{2}+\omega_{2}^{\prime}, \lambda_{3}=\omega_{2}^{\prime}, \lambda_{4}=\omega_{1}+\omega_{3}^{\prime}, \lambda_{5}=\omega_{2}+\omega_{1}^{\prime}+\omega_{3}^{\prime}, \lambda_{6}=\omega_{4}^{\prime}$. Then

$$
\begin{aligned}
& E=\left\{\lambda_{1}, \lambda_{2}, \lambda_{3}, \lambda_{4}, \lambda_{5}, \lambda_{6}\right\} ; \\
& d_{W}=5 .
\end{aligned}
$$

Proposition 3.10. The $T_{\mathrm{ad}}$-module $T_{X_{0}} \mathrm{M}_{\mathcal{S}}^{G}$ is multiplicity-free. Its $T_{\mathrm{ad}}$-weight set is

$$
\left\{\alpha_{1}, \alpha_{2}, \alpha_{1}^{\prime}, \alpha_{2}^{\prime}, \alpha_{3}^{\prime}\right\}
$$

In particular, $\operatorname{dim} T_{X_{0}} \mathrm{M}_{\mathcal{S}}^{G}=d_{W}$.

Proof. Let $\beta$ be a $T_{\text {ad }}$-weight in $T_{X_{0}} \mathrm{M}_{\mathcal{S}}^{G}$. It follows from Proposition 2.19 that at least one $\lambda \in E$ has a positive coefficient in the expression of $\beta$ as a $\mathbb{Z}$-linear combination of elements of $E$. Note that all elements of $E$ except $\lambda_{6}$ have codimension 1. In particular, if $\lambda \neq \lambda_{6}$, then it follows from Proposition 2.21 that $\beta$ is a simple root belonging to the set (3.20), and that its weight space has dimension one.

Consequently, to prove the proposition, what remains is to show that $\lambda_{6}$ cannot be the only element of $E$ which has a positive coefficient in the expression of $\beta$ as a linear combination of the elements of $E$.

Note that $\bar{G}^{\prime}=\operatorname{Sp}(4) \times \operatorname{SL}(n)$ and therefore that $\operatorname{dim} \bar{G} / \bar{G}^{\prime}=1$. For $n=4$ the only connected group between $\bar{G}^{\prime}$ and $\bar{G}$, for which $W$ is spherical, is $G=\bar{G}$. On the other hand, if $n>4$, then there are two such groups: $G=\bar{G}$ and $G=\bar{G}^{\prime}$; cf. Remark 3.3, Straightforward computations show that

$$
\langle E\rangle_{\mathbb{Z}} \cap \Lambda_{R}= \begin{cases}\left\langle\alpha_{1}, \alpha_{2}, \alpha_{1}^{\prime}, \alpha_{2}^{\prime}, \alpha_{3}^{\prime}\right\rangle_{\mathbb{Z}} & \text { if } G=\bar{G} \text { or } n \text { is odd or } n=4 ; \\ \left\langle\alpha_{1}, \alpha_{2}, \alpha_{1}^{\prime}, \alpha_{2}^{\prime}, \alpha_{3}^{\prime}, \gamma\right\rangle_{\mathbb{Z}} & \text { if } G=\bar{G}^{\prime} \text { and } n \text { is even and } n>4\end{cases}
$$

where

$$
\gamma=(n-4) \alpha_{4}^{\prime}+(n-5) \alpha_{5}^{\prime}+\ldots+2 \alpha_{n-2}^{\prime}+\alpha_{n-1}^{\prime} .
$$

Let $K$ be the basis of $\langle E\rangle_{\mathbb{Z}} \cap \Lambda_{R}$ in equation (3.21). Since $\beta \in\langle\Pi\rangle_{\mathbb{N}}$ and all elements of $K$ contain a simple root in their support, which is not in the support of any other element of $K$, it follows that $\beta \in\langle K\rangle_{\mathbb{N}}$. We have the following equalities:

$$
\begin{aligned}
\alpha_{1} & =\lambda_{1}+\lambda_{4}-\lambda_{5} ; \\
\alpha_{2} & =\lambda_{2}+\lambda_{5}-\lambda_{3}-\lambda_{1}-\lambda_{4} ; \\
\alpha_{1}^{\prime} & =\lambda_{1}+\lambda_{5}-\lambda_{4}-\lambda_{2} ; \\
\alpha_{2}^{\prime} & =\lambda_{2}+\lambda_{3}-\lambda_{5} ; \\
\alpha_{3}^{\prime} & =\lambda_{4}+\lambda_{5}-\lambda_{6}-\lambda_{1}-\lambda_{2} ; \\
\gamma & =(n-3) \lambda_{6}-\frac{n-4}{2}\left[\lambda_{4}+\lambda_{5}-\lambda_{1}-\lambda_{2}+\lambda_{3}\right] .
\end{aligned}
$$

As one easily sees, $\gamma$ is the only element of $K$ in which $\lambda_{6}$ has a positive coefficient. Consequently, the Proposition follows from equation (3.21) for $G=\bar{G}$, for odd $n$ and for $n=4$.

We now assume that $G=\bar{G}^{\prime}$, that $n$ is even and at least 6 and that $\lambda_{6}$ has a positive coefficient in $\beta$. We will come to a contradiction. Our assumptions imply that $\gamma$ has 
a positive coefficient in the expression of $\beta$ as an $\mathbb{N}$-linear combination of the elements of $K$. Recall from Proposition 2.6 that $\beta$ is the sum of a simple root and an element of $R^{+} \cup\{0\}$. By equation (3.22) this is only possible if $n=6$ and if $\beta$ is one of the following three elements of $\langle K\rangle_{\mathbb{N}}$ :

$$
\begin{aligned}
& \beta_{1}:=\alpha_{1}^{\prime}+\alpha_{2}^{\prime}+\alpha_{3}^{\prime}+\gamma ; \\
& \beta_{2}:=\alpha_{2}^{\prime}+\alpha_{3}^{\prime}+\gamma ; \\
& \beta_{3}:=\alpha_{3}^{\prime}+\gamma .
\end{aligned}
$$

Since $\beta_{1}=\lambda_{1}+2 \lambda_{6}-\lambda_{4}$ and $\beta_{2}=\lambda_{2}+2 \lambda_{6}-\lambda_{5}$, it follows that $\beta$ cannot be either of them by Proposition 2.20, Finally, $\beta=\beta_{3}$ is not possible because if $v \in V^{\beta_{3}}$ with $0 \neq[v] \in\left(V / \mathfrak{g} \cdot x_{0}\right)^{G_{x_{0}}}$, then it follows from Proposition 2.6 that $X_{\alpha_{4}^{\prime}} \cdot v$ is a nonzero element in $\left\langle X_{-\left(\beta_{3}-\alpha_{4}^{\prime}\right)} \cdot x_{0}\right\rangle_{C}$, since $\alpha_{4}^{\prime}$ is the only simple root such that $\beta_{3}-\alpha_{4}^{\prime} \in R^{+} \cup\{0\}$. Since $X_{-\left(\beta_{3}-\alpha_{4}^{\prime}\right)} \cdot x_{0}$ has nonzero projection on $V\left(\lambda_{4}\right)$, so does $v$, but $\beta_{3}$ does not occur as a $T_{\text {ad }}$-weight in $V\left(\lambda_{4}\right)$ as follows immediately from the well known list of $T$-weights in $V\left(\omega_{3}^{\prime}\right)$. This completes the proof.

3.6. (K9) The modules $\left(\mathrm{SO}(n) \times \mathbb{C}^{\times}, \mathbb{C}^{n}\right)$ with $3 \leq n$. For these modules

$$
\begin{aligned}
& E=\left\{\omega_{1}+\varepsilon, 2 \varepsilon\right\} \\
& d_{W}=1 .
\end{aligned}
$$

Proposition 3.11. The $T_{\mathrm{ad}}$-module $\left(V / \mathfrak{g} \cdot x_{0}\right)^{G_{x_{0}}^{\prime}}$ is one-dimensional. Its weight is

$$
\begin{array}{r}
2 \alpha_{1}+2 \alpha_{2}+\ldots+2 \alpha_{(n / 2)-2}+\alpha_{(n / 2)-1}+\alpha_{n / 2} \quad \text { if } n \text { is even; } \\
2 \alpha_{1}+2 \alpha_{2}+\ldots+2 \alpha_{(n-1) / 2} \quad \text { if } n \text { is odd. }
\end{array}
$$

In particular, $\operatorname{dim}\left(V / \mathfrak{g} \cdot x_{0}\right)^{G_{x_{0}}^{\prime}}=d_{W}$. Consequently, $\operatorname{dim} T_{X_{0}} \mathrm{M}_{\mathcal{S}}^{G}=d_{W}$.

Proof. Observe that $\operatorname{dim} V=\operatorname{dim} W+1$, since $V(2 \varepsilon)$ is one-dimensional. Since $\operatorname{dim} W=$ $\operatorname{dim} \mathfrak{g} \cdot x_{0}$ (by Lemma 3.5), this implies that $\operatorname{dim} V / \mathfrak{g} \cdot x_{0}=1$. Since $d_{W}=1$, this implies that $\operatorname{dim}\left(V / \mathfrak{g} \cdot x_{0}\right)^{G_{x_{0}}^{\prime}}=1$ by [PVS12, Proposition 2.5(1) and Corollary 2.14].

We now find the $T_{\text {ad }}$-weight of $\left(V / \mathfrak{g} \cdot x_{0}\right)^{G_{x_{0}}^{\prime}}$. Using the well-known $T$-weight space decomposition of the G-module $V\left(\omega_{1}+\varepsilon\right) \simeq \mathbb{C}^{n} \otimes \mathbb{C}_{\varepsilon}$, the fact that $V(2 \varepsilon) \subset \mathfrak{g} \cdot x_{0}$ and [PVS12, Lemma 2.16(4)], one readily checks that the one-dimensional $T_{\text {ad }}$-module $V / \mathfrak{g} \cdot x_{0}$ has the $T_{\mathrm{ad}}$-weight given in the proposition.

3.7. (K10) The module $\left(\operatorname{Spin}(10) \times \mathbb{C}^{\times}, \mathbb{C}^{16}\right)$. Here

$$
\begin{aligned}
& E=\left\{\omega_{5}+\varepsilon, \omega_{1}+2 \varepsilon\right\} ; \\
& d_{W}=1 .
\end{aligned}
$$

Proposition 3.12. The $T_{\mathrm{ad}}$-module $\left(V / \mathfrak{g} \cdot x_{0}\right)^{G_{x_{0}}^{\prime}}$ is one-dimensional. Its weight is

$$
\alpha_{2}+2 \alpha_{3}+\alpha_{4}+2 \alpha_{5} \text {. }
$$

In particular, $\operatorname{dim}\left(V / \mathfrak{g} \cdot x_{0}\right)^{G_{x_{0}}^{\prime}}=d_{W}$. Consequently, $\operatorname{dim} T_{X_{0}} \mathrm{M}_{\mathcal{S}}^{G}=d_{W}$. 
Proof. Recall that $p$ is the projection from the weight lattice $\Lambda$ of $G$ to the weight lattice of $G^{\prime}=\operatorname{Spin}(10)$. We first observe that $W=V\left(\omega_{5}+\varepsilon\right)$ is spherical for $G^{\prime}=\operatorname{Spin}(10)$ (cf. Remark 3.3) and that its weight monoid $p(\mathcal{S})$ is $G^{\prime}$-saturated. By [PVS12, Corollary 2.27] it follows that $\operatorname{dim}\left(V / \mathfrak{g} \cdot x_{0}\right)^{G_{x_{0}}^{\prime}}=d_{W}$.

While we do not need it for Theorem 1.2, we give a proof of the claim that the $T_{\text {ad }}{ }^{-}$ weight of $\left(V / \mathfrak{g} \cdot x_{0}\right)^{G_{x_{0}}^{\prime}}$ is $\alpha_{2}+2 \alpha_{3}+\alpha_{4}+2 \alpha_{5}$. A straightforward calculation shows that

$$
\begin{aligned}
p\left(\langle E\rangle_{\mathbb{Z}}\right) \cap \Lambda_{R} & =\left\langle 2 \alpha_{1}+\alpha_{2}-\alpha_{5},-2 \alpha_{1}+2 \alpha_{3}+\alpha_{4}+3 \alpha_{5}\right\rangle_{\mathbb{Z}} \\
& =\left\langle\beta_{1}, \beta_{2}\right\rangle_{\mathbb{Z}} .
\end{aligned}
$$

where $\beta_{1}=\alpha_{2}+2 \alpha_{3}+\alpha_{4}+2 \alpha_{5}$ and $\beta_{2}=2 \alpha_{1}+2 \alpha_{2}+2 \alpha_{3}+\alpha_{4}+\alpha_{5}$. If $\beta$ is a $T_{\text {ad }}$-weight occurring in $\left(V / \mathfrak{g} \cdot x_{0}\right)^{G_{x_{0}}^{\prime}}$, then, by Proposition 2.6 ,

$$
\begin{aligned}
& \beta \in\langle\Pi\rangle_{\mathbb{N}} \cap p\left(\langle E\rangle_{\mathbb{Z}}\right)=\langle\Pi\rangle_{\mathbb{N}} \cap\left\langle\beta_{1}, \beta_{2}\right\rangle_{\mathbb{Z}} ; \text { and } \\
& \beta \in \Pi+\left(R^{+} \cup\{0\}\right)
\end{aligned}
$$

In the root system of type $D_{5}$, if an element of $\Pi+\left(R^{+} \cup\{0\}\right)$ is written as a linear combination of the simple roots, then none of the coefficients are greater than 3 . Consequently, (3.25) and (3.26) imply that there exists $a, b \in \mathbb{Z}$ such that $\beta=a \beta_{1}+b \beta_{2}$ and

$$
\begin{aligned}
3 \geq 2 b & \geq 0 \\
3 \geq 2 a+2 b & \geq 0 \\
3 \geq 2 a+b & \geq 0
\end{aligned}
$$

It follows from (3.27) that $b \in\{0,1\}$. If $b=0$, then it follows from (3.28) that $a \in\{0,1\}$. If $b=1$, then it follows from (3.29) that $a \in\{0,1\}$, and then (3.28) implies that $a=0$. Since $\beta \neq 0$, we have shown that $\beta=\beta_{1}$ or $\beta=\beta_{2}$.

To finish the proof, we have to show that $\beta_{2}$ cannot occur as a $T_{\text {ad }}$-weight in $(V / \mathfrak{g}$. $\left.x_{0}\right)^{G_{x_{0}}^{\prime}}$. To get a contradiction, suppose that $v \in V^{\beta_{2}}$ such that $0 \neq[v] \in\left(V / \mathfrak{g} \cdot x_{0}\right)^{G_{x_{0}}^{\prime}}$. Since $\alpha=\alpha_{1}$ is the only simple root such that $\beta_{2}-\alpha \in R^{+} \cup\{0\}$, it follows from Proposition 2.6 that

$$
X_{\alpha_{1}} \cdot v \in\left\langle X_{-\left(\beta_{2}-\alpha_{1}\right)} \cdot x_{0}\right\rangle_{\mathbb{C}} \backslash\{0\}
$$

Since $\left\langle\left(\beta_{2}-\alpha_{1}\right)^{\vee}, \omega_{1}\right\rangle \neq 0$ and $\left\langle\left(\beta_{2}-\alpha_{1}\right)^{\vee}, \omega_{5}\right\rangle \neq 0$, the vector $X_{-\left(\beta_{2}-\alpha_{1}\right)} \cdot x_{0}$ has nonzero projection on both irreducible components of $V$. This is in contradiction with (3.30), since the $T_{\text {ad }}$-weight $\beta$ does not occur in $V\left(\omega_{5}\right)$. This finishes the proof.

Remark 3.13. The fact that the $T_{\text {ad }}$-weight of $\left(V / \mathfrak{g} \cdot x_{0}\right)^{G_{x_{0}}^{\prime}}$ is $\alpha_{2}+2 \alpha_{3}+\alpha_{4}+2 \alpha_{5}$, which is equal to $\varepsilon_{2}+\varepsilon_{3}+\varepsilon_{4}+\varepsilon_{5}$, can also be deduced from the description of the little Weyl group of the module $W^{*}$ given in [Kno98] (see [PVS12, Remark 2.8] for some context.)

3.8. (K11) The module $\left(\operatorname{Spin}(7) \times \mathbb{C}^{\times}, \mathbb{C}^{8}\right)$. Here

$$
\begin{aligned}
& E=\left\{\omega_{3}+\varepsilon, 2 \varepsilon\right\} \\
& d_{W}=1 .
\end{aligned}
$$

Proposition 3.14. The $T_{\mathrm{ad}}$-module $\left(V / \mathfrak{g} \cdot x_{0}\right)^{G_{x_{0}}^{\prime}}$ is one-dimensional. Its weight is

$$
\alpha_{1}+2 \alpha_{2}+3 \alpha_{3} \text {. }
$$


In particular, $\operatorname{dim}\left(V / \mathfrak{g} \cdot x_{0}\right)^{G_{x_{0}}^{\prime}}=d_{W}$. Consequently, $\operatorname{dim} T_{X_{0}} \mathrm{M}_{\mathcal{S}}^{G}=d_{W}$

Proof. The proof that $\operatorname{dim}\left(V / \mathfrak{g} \cdot x_{0}\right)^{G_{x_{0}}^{\prime}}=1$ is exactly like in the proof of Proposition 3.11. We now find the $T_{\text {ad }}$-weight of $\left(V / \mathfrak{g} \cdot x_{0}\right)^{G_{x_{0}}^{\prime}}$, also like in the proof of Proposition 3.11, Using the $T$-weight space decomposition of the G-module $V\left(\omega_{3}+\varepsilon\right)$ (which can be computed by hand or with $L i E$ [VLCL92]), the fact that $V(2 \varepsilon) \subset \mathfrak{g} \cdot x_{0}$ and [PVS12, Lemma $2.16(4)]$, one readily checks that the one-dimensional $T_{\text {ad }}$-module $V / \mathfrak{g} \cdot x_{0}$ has the $T_{\mathrm{ad}^{-}}$ weight given in the proposition.

3.9. (K12) The module $\left(\operatorname{Spin}(9) \times \mathbb{C}^{\times}, \mathbb{C}^{16}\right)$. Here

$$
\begin{aligned}
& E=\left\{\omega_{4}+\varepsilon, \omega_{1}+2 \varepsilon, 2 \varepsilon\right\} ; \\
& d_{W}=2 .
\end{aligned}
$$

Proposition 3.15. The $T_{\mathrm{ad}}$-module $\left(V / \mathfrak{g} \cdot x_{0}\right)^{G_{x_{0}}^{\prime}}$ is multiplicity-free and has $T_{\mathrm{ad}}$-weight set

$$
\left\{\alpha_{1}+\alpha_{2}+\alpha_{3}+\alpha_{4}, \alpha_{2}+2 \alpha_{3}+3 \alpha_{4}\right\} \text {. }
$$

In particular, $\operatorname{dim}\left(V / \mathfrak{g} \cdot x_{0}\right)^{G_{x_{0}}^{\prime}}=d_{W}$. Consequently, $\operatorname{dim} T_{X_{0}} \mathrm{M}_{\mathcal{S}}^{G}=d_{W}$

Proof. Observe that $G^{\prime}=\operatorname{Spin}(9)$. Consider the $G^{\prime}$-module $V^{\prime}:=V\left(\omega_{1}\right) \oplus V\left(\omega_{4}\right)$ and its element $x_{0}^{\prime}=v_{\omega_{1}}+v_{\omega_{4}}$. Observe that $G_{x_{0}}^{\prime}=G_{x_{0}^{\prime}}^{\prime}$. Since $V(2 \varepsilon)$ is one-dimensional, we have that $\left(V^{\prime} / \mathfrak{g}^{\prime} \cdot x_{0}^{\prime}\right)^{G_{x_{0}}^{\prime}} \simeq\left(V / \mathfrak{g} \cdot x_{0}\right)^{G_{x_{0}}^{\prime}}$ as $T_{\text {ad }}$-modules. Put $E^{\prime}=p(E)=\left\{\omega_{1}, \omega_{4}\right\}$.

Let $\beta$ be a $T_{\text {ad }}$-weight of $\left(V^{\prime} / \mathfrak{g}^{\prime} \cdot x_{0}^{\prime}\right)^{G_{x_{0}}^{\prime}}$. Then, by Proposition 2.6,

$$
\begin{aligned}
& \beta \in\left\langle E^{\prime}\right\rangle_{\mathbb{Z}} \cap\langle\Pi\rangle_{\mathbb{N}} \\
& \beta \in \Pi+\left(R^{+} \cup\{0\}\right)
\end{aligned}
$$

A straightforward computation shows that

$$
\left\langle E^{\prime}\right\rangle_{\mathbb{Z}} \cap\langle\Pi\rangle_{\mathbb{Z}}=\left\langle\beta_{1}, \beta_{2}\right\rangle_{\mathbb{Z}}
$$

where $\beta_{1}=\alpha_{1}+\alpha_{2}+\alpha_{3}+\alpha_{4}$ and $\beta_{2}=\alpha_{2}+2 \alpha_{3}+3 \alpha_{4}$. The explicit description of $R^{+}$ for Spin(9) (see, e.g. [Bou68, Planche II]) shows that (3.32) implies that if $\beta$ is written as a linear combination of the simple roots, then the coefficient of $\alpha_{1}$ is at most 2 and that of the other simple roots is at most 3. Combined with (3.31) and (3.33) this implies that there exist $a, b \in \mathbb{Z}$ such that $\beta=a \beta_{1}+b \beta_{2}$ and

$$
\begin{aligned}
2 \geq a & \geq 0 ; \text { and } \\
3 \geq a+3 b & \geq 0
\end{aligned}
$$

This system implies that $(a, b) \in\{(0,0),(1,0),(2,0),(0,1)\}$. Since $\beta \neq 0$, we have shown that

$$
\beta \in\left\{\alpha_{1}+\alpha_{2}+\alpha_{3}+\alpha_{4}, 2\left(\alpha_{1}+\alpha_{2}+\alpha_{3}+\alpha_{4}\right), \alpha_{2}+2 \alpha_{3}+3 \alpha_{4}\right\} .
$$

We claim that $\beta_{3}:=2\left(\alpha_{1}+\alpha_{2}+\alpha_{3}+\alpha_{4}\right)$ does not occur as a $T_{\text {ad }}$-weight in $\left(V^{\prime} / \mathfrak{g}^{\prime}\right.$. $\left.x_{0}^{\prime}\right)^{G_{x_{0}}^{\prime}}$. We will argue by contradiction; assume $v \in V^{\prime}$ is a $T_{\text {ad }}$-eigenvector of weight $\beta_{3}$ such that $[v]$ is nonzero in $\left(V^{\prime} / \mathfrak{g}^{\prime} \cdot x_{0}^{\prime}\right)^{G_{x_{0}}^{\prime}}$. Using the explicit description of $R^{+}$once more, 
one readily checks that $\alpha=\alpha_{1}$ is the only simple root $\alpha$ such that $\beta_{3}-\alpha \in R^{+} \cup\{0\}$. By Proposition 2.6 this implies that

$$
X_{\alpha_{1}} \cdot v \in\left\langle X_{-\left(\beta_{3}-\alpha_{1}\right)} \cdot x_{0}^{\prime}\right\rangle_{C} \backslash\{0\} .
$$

Because $\left\langle\left(\beta_{3}-\alpha_{1}\right)^{\vee}, \omega_{1}\right\rangle \neq 0$ and $\left\langle\left(\beta_{3}-\alpha_{1}\right)^{\vee}, \omega_{4}\right\rangle \neq 0$, we have that $X_{-\left(\beta_{3}-\alpha_{1}\right)} x_{0}^{\prime}$ has nonzero projection to both summands $V\left(\omega_{1}\right)$ and $V\left(\omega_{4}\right)$ of $V^{\prime}$. On the other hand, the following computation in $L i E$ shows that $\beta_{3}$ does not occur as a $T_{\text {ad }}$-weight in $V\left(\omega_{4}\right)$.

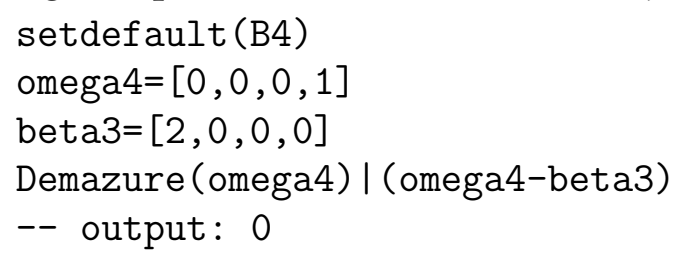

This implies that $v$ is in the kernel of the projection onto the summand $V\left(\omega_{4}\right)$ of $V^{\prime}$, which is in contradiction with equation (3.35). This proves the claim.

Since the monoid $\left\langle E^{\prime}\right\rangle_{\mathbb{N}}$ is free and $G^{\prime}$-saturated, we know that the $T_{\text {ad }}$-module $\left(V^{\prime} / \mathfrak{g}^{\prime}\right.$. $\left.x_{0}^{\prime}\right)^{G_{x_{0}}^{\prime}}$ is multiplicity-free by [BCF08, Theorem 3.10]. Equation (3.34) and the claim above

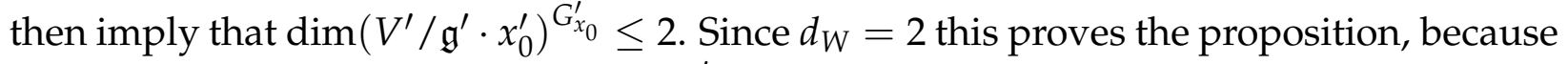
$T_{X_{0}} \mathrm{M}_{\mathcal{S}}^{G} \subseteq\left(V / \mathfrak{g} \cdot x_{0}\right)^{G_{x_{0}}} \subseteq\left(V^{\prime} / \mathfrak{g}^{\prime} \cdot x_{0}^{\prime}\right)^{G_{x_{0}}^{\prime}}$ and $\operatorname{dim} T_{X_{0}} \mathrm{M}_{\mathcal{S}}^{G} \geq d_{W}$, by [PVS12, Proposition 2.5(1)].

3.10. (K13) The module $\left(G_{2} \times \mathbb{C}^{\times}, \mathbb{C}^{7}\right)$. Here

$$
\begin{aligned}
& E=\left\{\omega_{1}+\varepsilon, 2 \varepsilon\right\} \\
& d_{W}=1 .
\end{aligned}
$$

Proposition 3.16. The $T_{\mathrm{ad}}$-module $\left(V / \mathfrak{g} \cdot x_{0}\right)^{G_{x_{0}}^{\prime}}$ is one-dimensional. Its weight is

$$
4 \alpha_{1}+2 \alpha_{2}
$$

In particular, $\operatorname{dim}\left(V / \mathfrak{g} \cdot x_{0}\right)^{G_{x_{0}}^{\prime}}=d_{W}$. Consequently, $\operatorname{dim} T_{X_{0}} \mathbf{M}_{\mathcal{S}}^{G}=d_{W}$

Proof. Same argument as for Proposition 3.14 .

3.11. (K14) The module $\left(E_{6} \times \mathbb{C}^{\times}, \mathbb{C}^{27}\right)$. Here

$$
\begin{aligned}
& E=\left\{\omega_{1}+\varepsilon, \omega_{6}+2 \varepsilon, 3 \varepsilon\right\} \\
& d_{W}=2 .
\end{aligned}
$$

Proposition 3.17. The $T_{\mathrm{ad}}$-module $\left(V / \mathfrak{g} \cdot x_{0}\right)^{G_{x_{0}}^{\prime}}$ is multiplicity-free and has $T_{\mathrm{ad}}$-weight set

$$
\left\{\alpha_{2}+\alpha_{3}+2 \alpha_{4}+2 \alpha_{5}+2 \alpha_{6}, 2 \alpha_{1}+\alpha_{2}+2 \alpha_{3}+2 \alpha_{4}+\alpha_{5}\right\} \text {. }
$$

In particular, $\operatorname{dim}\left(V / \mathfrak{g} \cdot x_{0}\right)^{G_{x_{0}}^{\prime}}=d_{W}$. Consequently, $\operatorname{dim} T_{X_{0}} \mathrm{M}_{\mathcal{S}}^{G}=d_{W}$

Proof. Note that $G^{\prime}=\mathrm{E}_{6}$. Consider the $G^{\prime}$-module $V^{\prime}:=V\left(\omega_{1}\right) \oplus V\left(\omega_{6}\right)$ and its element $x_{0}^{\prime}=v_{\omega_{1}}+v_{\omega_{6}}$. Since $V(3 \varepsilon) \subseteq \mathfrak{g} \cdot x_{0}$, we have that $\left(V^{\prime} / \mathfrak{g}^{\prime} \cdot x_{0}^{\prime}\right)^{G_{x_{0}}^{\prime}} \simeq\left(V / \mathfrak{g} \cdot x_{0}\right)^{G_{x_{0}}^{\prime}}$ as $T_{\text {ad }}$-modules. Observe that $G_{x_{0}}^{\prime}=G_{x_{0}^{\prime}}^{\prime}$,

The monoid $p\left(\langle E\rangle_{\mathbb{N}}\right)=\left\langle\omega_{1}, \omega_{6}\right\rangle_{\mathbb{N}}$ is free and $G^{\prime}$-saturated. By [BCF08, Theorems 3.1 and 3.10], $\left(V^{\prime} / \mathfrak{g}^{\prime} \cdot x_{0}^{\prime}\right)^{G_{x_{0}}^{\prime}}$ is multiplicity-free and its $T_{\text {ad }}$-weights belong to Table 1 in 
[BCF08, page 2810]. By Proposition 2.6 the $T_{\text {ad }}$-weights of $\left(V^{\prime} / \mathfrak{g}^{\prime} \cdot x_{0}^{\prime}\right)^{G_{x_{0}}^{\prime}}$ also belong to $\Lambda_{R} \cap\left\langle\omega_{1}, \omega_{6}\right\rangle_{\mathbb{Z}}$. A straightforward computation shows that

$$
\Lambda_{R} \cap\left\langle\omega_{1}, \omega_{6}\right\rangle_{\mathbb{Z}}=\left\langle\alpha_{2}+\alpha_{3}+2 \alpha_{4}+2 \alpha_{5}+2 \alpha_{6}, 2 \alpha_{1}+\alpha_{2}+2 \alpha_{3}+2 \alpha_{4}+\alpha_{5}\right\rangle_{\mathbb{Z}}
$$

Observe that the support of each of the two generators of $\Lambda_{R} \cap\left\langle\omega_{1}, \omega_{6}\right\rangle_{\mathbb{Z}}$ in equation (3.36) contains a simple root not in the support of the other generator. Because the $T_{\text {ad }}$-weights of $\left(V^{\prime} / \mathfrak{g}^{\prime} \cdot x_{0}^{\prime}\right)^{G_{x_{0}}^{\prime}}$ belong to $\langle\Pi\rangle_{\mathbb{N}}$, it follows that they belong to $\left\langle\alpha_{2}+\alpha_{3}+2 \alpha_{4}+2 \alpha_{5}+\right.$ $\left.2 \alpha_{6}, 2 \alpha_{1}+\alpha_{2}+2 \alpha_{3}+2 \alpha_{4}+\alpha_{5}\right\rangle_{\mathbb{N}}$. Because none of the $T_{\text {ad }}$-weights in [BCF08, Table 1] supported on a subdiagram of $\mathrm{E}_{6}$ has a coefficient greater than 2, it follows that the $T_{\text {ad }}-$ weights of $\left(V^{\prime} / \mathfrak{g}^{\prime} \cdot x_{0}^{\prime}\right)^{G_{x_{0}}^{\prime}}$ are a subset of $\left\{\alpha_{2}+\alpha_{3}+2 \alpha_{4}+2 \alpha_{5}+2 \alpha_{6}, 2 \alpha_{1}+\alpha_{2}+2 \alpha_{3}+2 \alpha_{4}+\right.$ $\alpha_{5}$ \}. Since $d_{W}=2$, this proves the proposition.

3.12. (K19) The module $\left(\operatorname{Sp}(2 n) \times \mathbb{C}^{\times} \times \mathbb{C}^{\times}, \mathbb{C}^{2 n} \oplus \mathbb{C}^{2 n}\right)$ with $2 \leq n$. For these modules,

$$
\begin{aligned}
& E=\left\{\lambda_{1}, \lambda_{2}, \lambda_{3}, \lambda_{4}\right\} ; \\
& d_{W}=2,
\end{aligned}
$$

where

$$
\begin{array}{ll}
\lambda_{1}:=\omega_{1}+\varepsilon ; & \lambda_{2}:=\omega_{1}+\varepsilon^{\prime} ; \\
\lambda_{3}:=\omega_{2}+\varepsilon+\varepsilon^{\prime} ; & \lambda_{4}:=\varepsilon+\varepsilon^{\prime} .
\end{array}
$$

Note that $G=\bar{G}$ is the only connected group between $\bar{G}^{\prime}$ and $\bar{G}$ for which this module is spherical, cf. Remark 3.3. Therefore, we can assume throughout this section that $G=$ $\bar{G}=\operatorname{Sp}(2 n) \times \mathbb{C}^{\times} \times \mathbb{C}^{\times}$.

Proposition 3.18. The $T_{\mathrm{ad}}$-module $T_{X_{0}} \mathrm{M}_{\mathcal{S}}^{G}$ is multiplicity-free and has $T_{\mathrm{ad}}$-weight set

$$
\left\{\alpha_{1}, \alpha_{1}+\gamma\right\}
$$

where $\gamma=2\left(\alpha_{2}+\alpha_{3}+\ldots+\alpha_{n-1}\right)+\alpha_{n}$ if $n>2$ and $\gamma=\alpha_{2}$ if $n=2$. In particular, $\operatorname{dim} T_{X_{0}} \mathrm{M}_{\mathcal{S}}^{G}=d_{W}$.

Proof. This proof is similar to that of Proposition 3.8. Let $\beta$ be a $T_{\text {ad }}$-weight in $T_{X_{0}} \mathrm{M}_{\mathcal{S}}^{G}$. By Proposition 2.6, we know that $\beta \in\langle\Pi\rangle_{\mathbb{N}} \cap\langle E\rangle_{Z}$. A straightforward computation shows that $\Lambda_{R} \cap\langle E\rangle_{\mathbb{Z}}=\left\langle\alpha_{1}, \gamma\right\rangle_{\mathbb{Z}}$. Since $\alpha_{1}$ is not in the support of $\gamma$ and $\alpha_{2}$ is in the support of $\gamma$ but not in the support of $\alpha_{1}$, it follows that

$$
\beta \in\left\langle\alpha_{1}, \gamma\right\rangle_{\mathbb{N}}
$$

By Proposition 2.19, at least one element of $\left\{\lambda_{1}, \lambda_{2}, \lambda_{3}\right\}$ must have a positive coefficient in the expression of $\beta$ as a linear combination of the elements of $E$. Since $\lambda_{1}$ and $\lambda_{2}$ have codimension 1, it follows from Proposition 2.21 that if one of them has a positive coefficient, then $\beta=\alpha_{1}$ and $\beta$ has multiplicity one in $T_{X_{0}} \mathrm{M}_{\mathcal{S}}^{G}$. To finish the proof it is therefore enough to show the following four claims:

Claim A: if $\lambda_{3}$ is the only element of $E$ which has a positive coefficient in the expression of $\beta$ as a linear combination of the elements of $E$, then $\beta \in\left\{\alpha_{1}+\gamma, \gamma\right\}$ if $n>2$ and $\beta \in\left\{\alpha_{1}+\gamma, \gamma, 2 \alpha_{1}+2 \gamma\right\}$ if $n=2$.

Claim B: the $T_{\text {ad }}$-weight $\beta=\gamma$ does not occur in $\left(V / \mathfrak{g} \cdot x_{0}\right)^{G_{x_{0}}}$, and therefore also not in the subspace $T_{X_{0}} \mathrm{M}_{\mathcal{S}}^{G}$. 
Claim C: suppose $n=2$; the $T_{\text {ad }}$-weight $\beta=2 \alpha_{1}+2 \gamma$ does not occur in $(V / \mathfrak{g}$. $\left.x_{0}\right)^{G_{x_{0}}}$, and therefore also not in the supspace $T_{X_{0}} \mathrm{M}_{\mathcal{S}}^{G}$.

Claim D: the $T_{\text {ad }}$-weight $\beta=\alpha_{1}+\gamma$ has multiplicity at most one in $T_{X_{0}} \mathrm{M}_{\mathcal{S}}^{G}$.

To prove Claim A, we first observe that

$$
\begin{aligned}
\alpha_{1} & =\lambda_{1}+\lambda_{2}-\lambda_{3} \\
\gamma & =2 \lambda_{3}-\lambda_{1}-\lambda_{2}-\lambda_{4} .
\end{aligned}
$$

It follows from (3.37) and the hypothesis of Claim $\mathrm{A}$, that there exist $A, B \in \mathbb{N}$ with $B>0$ and $B \geq A$ such that

$$
\beta=A \alpha_{1}+B \gamma
$$

For $n>2$ the only $\beta$ as in (3.38) that satisfy Proposition 2.6(a) are $\gamma$ and $\alpha_{1}+\gamma$. For $n=2$, there are three additional such $\beta$, namely $\beta=2 \gamma=2 \alpha_{2}, \beta=\alpha_{1}+2 \gamma=\alpha_{1}+2 \alpha_{2}$ and $\beta=2 \alpha_{1}+2 \alpha_{2}$. Proposition 2.20 tells us that $\beta=2 \alpha_{2}$ and $\beta=\alpha_{1}+2 \alpha_{2}$ cannot occur as a $T_{\mathrm{ad}}$-weight in $T_{X_{0}} \mathbf{M}_{\mathcal{S}}^{G}$. This finishes the proof of Claim $\mathrm{A}$.

We proceed to Claim B. Let $\beta=\gamma$. Observe that the $T_{\text {ad }}$-weight $\gamma$ does not occur in the G-modules $V\left(\lambda_{1}\right), V\left(\lambda_{2}\right)$ and $V\left(\lambda_{4}\right)$. It occurs in $V\left(\lambda_{3}\right)$ with multiplicity one: the $T_{a^{-}}$ weight space in $V\left(\lambda_{3}\right)$ of weight $\beta$ is spanned by $X_{-\beta} v_{\lambda_{3}}$. It follows that $\beta$ occurs with multiplicity one in $V$ and that its weight space is a subspace of $\mathfrak{g} \cdot x_{0}$. This proves Claim B.

We move to Claim C. Let $n=2$ and $\beta=2 \alpha_{1}+2 \alpha_{2}$. One deduces from the wellknown decompositions into T-weight spaces of $V\left(\omega_{1}\right)$ and $V\left(\omega_{2}\right)$ that the $T_{\text {ad }}$-weight space in $V$ of $T_{\text {ad }}$-weight $\beta$ is a line $\mathbb{C} v$ in $V\left(\lambda_{3}\right) \subseteq V$. We prove Claim C by contradiction. Indeed, since $\alpha_{2}$ is the only simple root such that $\beta-\alpha_{2} \in R^{+} \cup\{0\}$, we have that $0 \neq$ $[v] \in\left(V / \mathfrak{g} \cdot x_{0}\right)^{G_{x_{0}}}$ would, by Proposition 2.6, imply that $X_{\alpha_{2}} \cdot v$ is a nonzero element of $\left\langle X_{-\left(\beta-\alpha_{2}\right)} \cdot x_{0}\right\rangle_{C}$. This is absurd since $X_{-\left(\beta-\alpha_{2}\right)} \cdot x_{0}$ has nonzero projection onto the components $V\left(\lambda_{1}\right)$ and $V\left(\lambda_{2}\right)$ of $V$. Claim $C$ is proved.

Finally, we show Claim D. We fix $\beta=\alpha_{1}+\gamma$. We will show that the $T_{\text {ad }}$-weight $\beta$ has multiplicity at most one in $\left(V / \mathfrak{g} \cdot x_{0}\right)^{G_{x_{0}}}$. Since $T_{X_{0}} \mathrm{M}_{\mathcal{S}}^{G} \subseteq\left(V / \mathfrak{g} \cdot x_{0}\right)^{G_{x_{0}}}$, this implies Claim D. First off, we observe that the $T_{\text {ad }}$-weight $\beta$ can only occur in $V\left(\lambda_{1}\right), V\left(\lambda_{2}\right)$ or $V\left(\lambda_{3}\right)$. Let $Z$ be the subspace of $V\left(\lambda_{1}\right) \oplus V\left(\lambda_{2}\right) \oplus V\left(\lambda_{3}\right)$ consisting of $T_{\text {ad }}$-eigenvectors $v$ of $T_{\text {ad }}$-weight $\beta$ that satisfy the following three conditions:

$$
\begin{aligned}
& X_{\alpha_{1}} \cdot v \in\left\langle X_{-\left(\beta-\alpha_{1}\right)} x_{0}\right\rangle_{\mathbb{C}} ; \\
& X_{\alpha_{2}} \cdot v \in\left\langle X_{-\left(\beta-\alpha_{2}\right)} x_{0}\right\rangle_{\mathbb{C}} ; \text { and } \\
& X_{\alpha_{k}} \cdot v=0 \text { for all } k \in\{1,2, \ldots, n\} \backslash\{1,2\} .
\end{aligned}
$$

By Proposition 2.6, every $v \in V^{\beta}$ with $0 \neq[v] \in\left(V / \mathfrak{g} \cdot x_{0}\right)^{G_{x_{0}}}$ satisfies (3.39), (3.40) and (3.41). To show Claim $D$ it is therefore enough to prove that

$$
\operatorname{dim} Z \leq 2,
$$

since the nonzero vector $X_{-\beta} \cdot x_{0}$ belongs to $\mathfrak{g} \cdot x_{0} \cap \mathrm{Z}$. 
To prove the inequality (3.42) we will make use of the explicit description of $\mathfrak{s p}(2 n)$ and its root operators given in the proof of [GW09, Theorem 2.4.1], as well as the notations therein like we did in the proof of Proposition 3.8; see page 21.

Note that

$$
\beta=\alpha_{1}+\gamma=\omega_{2}=\varepsilon_{1}+\varepsilon_{2} .
$$

We now identify the weight spaces of this $T_{\text {ad }}$-weight in the representations $V\left(\omega_{1}\right)$ and $V\left(\omega_{2}\right)$ of $\operatorname{Sp}(2 n)$. A vector in $V\left(\omega_{1}\right)$ has $T_{\text {ad }}$-weight $\beta$ if and only if it has $T$-weight $\omega_{1}-$ $\beta=-\varepsilon_{2}$. We identify $V\left(\omega_{1}\right)$ with the standard representation $\mathbb{C}^{2 n}$ of $\operatorname{Sp}(2 n)$, which has $e_{1}$ as a highest weight vector. Then the $T$-weight space of weight $-\varepsilon_{2}$ is the line spanned by $e_{-2}$.

A vector in $V\left(\omega_{2}\right)$ has $T_{\text {ad }}$-weight $\beta$ if and only if it has $T$-weight $\omega_{2}-\beta=0$. As is well-known, $V\left(\omega_{2}\right)$ is the irreducible component of the $\operatorname{Sp}(2 n)$-module $\wedge^{2} \mathbb{C}^{2 n}$ generated by the highest weight vector $e_{1} \wedge e_{2}$. In the larger module $\wedge^{2} \mathbb{C}^{2 n}$ the $T$-weight space of weight 0 is spanned by the following vectors

$$
e_{1} \wedge e_{-1}, e_{2} \wedge e_{-2}, \ldots, e_{n} \wedge e_{-n} .
$$

It follows from the previous paragraph that if $v \in Z$, then there exist $A, B \in \mathbb{C}$ and $C_{1}, C_{2}, \ldots, C_{n} \in \mathbb{C}$ such that

$$
v=A\left(e_{-2} \otimes v_{\mathcal{E}}\right)+B\left(e_{-2} \otimes v_{\mathcal{E}^{\prime}}\right)+\sum_{k=1}^{n} C_{k}\left(e_{k} \wedge e_{-k} \otimes v_{\mathcal{E}+\varepsilon^{\prime}}\right) .
$$

Straightforward computations using the root operators show that conditions (3.39), (3.40) and (3.41) imply that when $n>2$,

$$
\begin{aligned}
& A=B=C_{3}-C_{2} ; \text { and } \\
& C_{3}=C_{4}=\ldots=C_{n},
\end{aligned}
$$

and that when $n=2$,

$$
A=B
$$

Either way, this implies that $\operatorname{dim} Z \leq 3$. Note that the vector $v_{1} \otimes v_{\varepsilon+\varepsilon^{\prime}} \in \wedge^{2} \mathbb{C}^{2 n} \otimes V(\varepsilon+$ $\left.\varepsilon^{\prime}\right)$, where

$$
v_{1}=\sum_{k=1}^{n} e_{k} \wedge e_{-k}
$$

satisfies the equations (3.45) and (3.46). It is straightforward to check that $v_{1}$ is a highest weight vector. It follows that $v_{1}$ is an element of the $\operatorname{Sp}(2 n)$-stable complement to $V\left(\omega_{2}\right)$ in $\wedge^{2} \mathbb{C}^{2 n}$. Consequently, the line spanned by $v_{1} \otimes v_{\varepsilon+\varepsilon^{\prime}}$ is not contained in $Z$, and $\operatorname{dim} Z \leq$ 2. This proves Claim $\mathrm{D}$, and the proposition.

Remark 3.19. Proceeding as in the proof of [PVS12, Proposition 5.14], one can show that in fact $\left(V / \mathfrak{g} \cdot x_{0}\right)^{G_{x_{0}}^{\prime}}$ is a multiplicity-free $T_{\text {ad }}$-module with the same $T_{\text {ad }}$-weight set as $T_{X_{0}} \mathrm{M}_{\mathcal{S}}$. 
3.13. (K20) The modules $\left(\left(\operatorname{Sp}(2 n) \times \mathbb{C}^{\times}\right) \times \mathrm{GL}(2),\left(\mathbb{C}^{2 n} \otimes \mathbb{C}^{2}\right) \oplus \mathbb{C}^{2}\right)$ with $2 \leq n$. For these modules,

$$
\begin{aligned}
& E=\left\{\lambda_{1}, \lambda_{2}, \lambda_{3}, \lambda_{4}, \lambda_{5}\right\} ; \\
& d_{W}=3,
\end{aligned}
$$

where

$$
\begin{array}{lll}
\lambda_{1}:=\omega_{1}^{\prime} ; & \lambda_{2}:=\omega_{1}+\varepsilon+\omega_{1}^{\prime} ; & \lambda_{3}:=\omega_{1}+\varepsilon+\omega_{2}^{\prime} ; \\
\lambda_{4}:=\omega_{2}+2 \varepsilon+\omega_{2}^{\prime} ; & \lambda_{5}:=2 \varepsilon+\omega_{2}^{\prime} . &
\end{array}
$$

We remark that $G=\bar{G}$ is the only connected group between $\bar{G}^{\prime}$ and $\bar{G}$ for which these modules are spherical, cf. Remark 3.3. Therefore, we assume $G=\bar{G}=\operatorname{Sp}(2 n) \times \mathbb{C}^{\times} \times$ GL(2) throughout this section.

In this section we will prove the following proposition.

Proposition 3.20. The $T_{\mathrm{ad}}$-module $T_{X_{0}} \mathrm{M}_{\mathcal{S}}^{G}$ is multiplicity-free. Its $T_{\mathrm{ad}}$-weight set is

$$
\left\{\alpha_{1}, \alpha_{1}+\gamma, \alpha_{1}^{\prime}\right\} \text {, }
$$

where $\gamma_{1}=\alpha_{2}$ if $n=2$ and $\gamma=2\left(\alpha_{2}+\alpha_{3}+\ldots+\alpha_{n-1}\right)+\alpha_{n}$ if $n>2$. In particular, $\operatorname{dim} T_{X_{0}} \mathrm{M}_{\mathcal{S}}^{G}=d_{W}$.

Proof. The argument is very similar to that of Proposition 3.8. Let $\beta$ be a $T_{\text {ad }}$-weight in $T_{X_{0}} \mathrm{M}_{\mathcal{S}}^{G}$. It follows from Proposition 2.19 that at least one $\lambda \in E$ has a positive coefficient in the expression of $\beta$ as a $\mathbb{Z}$-linear combination of elements of $E$. Note that all elements of $E$ except $\lambda_{4}$ have codimension 1. In particular, if $\lambda \neq \lambda_{4}$, then it follows from Proposition 2.21 that $\beta$ is a simple root belonging to the set (3.48), and that its weight space has dimension one. Consequently, to prove the proposition, what remains is to show the following two claims:

Claim A: if $\lambda_{4}$ is the only element of $E$ which has a positive coefficient in the expression of $\beta$, then $\beta=\alpha_{1}+\gamma$.

Claim B: the $T_{\text {ad }}$-weight $\beta=\alpha_{1}+\gamma$ has multiplicity at most one in $T_{X_{0}} \mathrm{M}_{\mathcal{S}}^{G}$.

We begin with Claim A. Recall from Proposition 2.6 that $\beta \in\langle E\rangle_{\mathbb{Z}} \cap\langle\Pi\rangle_{\mathbb{N}}$. Straightforward computations show that

$$
\langle E\rangle_{\mathbb{Z}} \cap \Lambda_{R}=\left\langle\alpha_{1}, \alpha_{1}^{\prime}, \gamma\right\rangle_{\mathbb{Z}}
$$

and that

$$
\begin{aligned}
\alpha_{1} & =\lambda_{2}+\lambda_{3}-\lambda_{1}-\lambda_{4} ; \\
\alpha_{1}^{\prime} & =\lambda_{1}+\lambda_{2}-\lambda_{3} ; \\
\gamma & =2 \lambda_{4}+\lambda_{1}-\lambda_{2}-\lambda_{3}-\lambda_{5} .
\end{aligned}
$$

Let $K$ be the basis of $\langle E\rangle_{\mathbb{Z}} \cap \Lambda_{R}$ given in equation (3.49). Since $\beta \in\langle\Pi\rangle_{\mathbb{N}}$ and all elements of $K$ contain a simple root in their support, which is not in the support of any other element of $K$, it follows that $\beta \in\langle K\rangle_{\mathbb{N}}$. Therefore, there exist $A, B, C \in \mathbb{N}$ such that

$$
\beta=A \alpha_{1}+B \alpha_{1}^{\prime}+C \gamma \text {. }
$$


From the hypothesis of Claim A, it follows that

$$
\left\{\begin{array}{l}
-A+B+C \leq 0 \\
A+B-C \leq 0 \\
A-B-C \leq 0
\end{array}\right.
$$

Adding the first two inequalities in (3.51) yields that $B=0$. After substituting $B=0$, the first two inequalities yield that $A=C$. It follows that $\beta \in\left\langle\alpha_{1}+\gamma\right\rangle_{\mathbb{N}}$. Using that $\beta$ is the sum of a simple root and an element of $R^{+} \cup\{0\}$ (see Proposition 2.6) it follows that $\beta=\alpha_{1}+\gamma$ when $n>2$ and that $\beta \in\left\{\alpha_{1}+\gamma, 2 \alpha_{1}+2 \gamma\right\}$ when $n=2$. With an argument like that for Claim $C$ in the proof of Proposition 3.18, one shows that $2 \alpha_{1}+2 \gamma$ cannot occur as a $T_{\text {ad }}$-weight in $\left(V / \mathfrak{g} \cdot x_{0}\right)^{G_{x_{0}}}$ when $n=2$. This proves Claim A.

The argument for Claim B is the same as that for Claim D in Proposition 3.18 above.

3.14. (K22) The modules $\left(\left(\operatorname{Sp}(2 m) \times \mathbb{C}^{\times}\right) \times \operatorname{SL}(2) \times \mathrm{GL}(n),\left(\mathbb{C}^{2 m} \otimes \mathbb{C}^{2}\right) \oplus\left(\mathbb{C}^{2} \otimes \mathbb{C}^{n}\right)\right)$ with $2 \leq m, n$. For these modules,

$$
\begin{aligned}
& E=\left\{\lambda_{1}, \lambda_{2}, \lambda_{3}, \lambda_{4}, \lambda_{5}, \lambda_{6}\right\} \\
& d_{W}=4
\end{aligned}
$$

where

$$
\begin{array}{lll}
\lambda_{1}:=\omega_{1}+\varepsilon+\omega^{\prime} ; & \lambda_{2}:=\omega^{\prime}+\omega_{1}^{\prime \prime} ; & \lambda_{3}:=\omega_{1}+\varepsilon+\omega_{1}^{\prime \prime} ; \\
\lambda_{4}:=\omega_{2}+2 \varepsilon ; & \lambda_{5}:=\omega_{2}^{\prime \prime} ; & \lambda_{6}:=2 \varepsilon .
\end{array}
$$

In this section we will prove the following proposition.

Proposition 3.21. The $T_{\mathrm{ad}}$-module $T_{X_{0}} \mathrm{M}_{\mathcal{S}}^{G}$ is multiplicity-free. Its $T_{\mathrm{ad}}$-weight set is

$$
\left\{\alpha_{1}, \alpha_{1}+\gamma, \alpha^{\prime}, \alpha_{1}^{\prime \prime}\right\},
$$

where $\gamma=\alpha_{2}$ if $m=2$ and $\gamma=2\left(\alpha_{2}+\alpha_{3}+\ldots+\alpha_{m-1}\right)+\alpha_{m}$ if $m>2$. In particular, $\operatorname{dim} T_{X_{0}} \mathrm{M}_{\mathcal{S}}^{G}=d_{W}$.

Proof. The argument is very similar to that of Propositions 3.8, 3.18 and 3.20, Let $\beta$ be a $T_{\text {ad }}$-weight in $T_{X_{0}} \mathrm{M}_{\mathcal{S}}^{G}$. It follows from Proposition 2.19 that at least one $\lambda \in E$ has a positive coefficient in the expression of $\beta$ as a $\mathbb{Z}$-linear combination of elements of $E$. Note that all elements of $E$ except $\lambda_{4}$ and $\lambda_{5}$ have codimension 1. In particular, if $\lambda \notin\left\{\lambda_{4}, \lambda_{5}\right\}$, then it follows from Proposition 2.21) that $\beta$ is a simple root belonging to the set (3.52), and that its weight space has dimension one.

Consequently, to prove the proposition, what remains is to show the following two claims:

Claim A: if $\lambda_{4}$ or $\lambda_{5}$ are the only elements of $E$ which have a positive coefficient in the expression of $\beta$, then $\beta=\alpha_{1}+\gamma$.

Claim B: the $T_{\text {ad }}$-weight $\beta=\alpha_{1}+\gamma$ has multiplicity at most one in $T_{X_{0}} \mathrm{M}_{\mathcal{S}}^{G}$.

We begin with Claim A. Recall from Proposition 2.6 that $\beta \in\langle E\rangle_{\mathbb{Z}} \cap\langle\Pi\rangle_{\mathbb{N}}$. Consequently $\beta \in p\left(\langle E\rangle_{\mathbb{Z}}\right) \cap \Lambda_{R}$. Straightforward computations show that

$$
p\left(\langle E\rangle_{\mathbb{Z}}\right) \cap \Lambda_{R}=\left\langle\alpha_{1}, \alpha^{\prime} \alpha_{1}^{\prime \prime}, \gamma\right\rangle_{\mathbb{Z}}
$$


and that

$$
\begin{aligned}
\alpha_{1} & =\lambda_{1}+\lambda_{3}-\lambda_{2}-\lambda_{4} \\
\alpha^{\prime} & =\lambda_{1}+\lambda_{2}-\lambda_{3} \\
\alpha_{1}^{\prime \prime} & =\lambda_{2}+\lambda_{3}-\lambda_{1}-\lambda_{5} \\
\gamma & =2 \lambda_{4}+\lambda_{2}-\lambda_{1}-\lambda_{3}-\lambda_{6} .
\end{aligned}
$$

Let $K$ be the basis of $p\left(\langle E\rangle_{\mathbb{Z}}\right) \cap \Lambda_{R}$ given in equation (3.53). Since $\beta \in\langle\Pi\rangle_{\mathbb{N}}$ and all elements of $K$ contain a simple root in their support, which is not in the support of any other element of $K$, it follows that $\beta \in\langle K\rangle_{\mathbb{N}}$. Therefore, there exist $A_{1}, A_{2}, A_{3}, A_{4} \in \mathbb{N}$ such that

$$
\beta=A_{1} \alpha_{1}+A_{2} \gamma+A_{3} \alpha^{\prime}+A_{4} \alpha_{1}^{\prime \prime} .
$$

From the hypothesis of Claim A, it follows that

$$
\left\{\begin{array}{l}
A_{1}-A_{2}+A_{3}-A_{4} \leq 0 \\
-A_{1}+A_{2}+A_{3}+A_{4} \leq 0 \\
A_{1}-A_{2}-A_{3}+A_{4} \leq 0
\end{array}\right.
$$

Adding the last two inequalities in (3.55) yields that $A_{4}=0$. After substituting $A_{4}=0$, the first two inequalities yield that $A_{3}=0$ and then that $A_{1}=A_{2}$. It follows that $\beta \in$ $\left\langle\alpha_{1}+\gamma\right\rangle_{\mathbb{N}}$. Using that $\beta$ is the sum of a simple root and an element of $R^{+} \cup\{0\}$ (see Proposition (2.6) it follows that $\beta=\alpha_{1}+\gamma$ when $m>2$ and that $\beta \in\left\{\alpha_{1}+\gamma, 2 \alpha_{1}+2 \gamma\right\}$ when $m=2$. With an argument like that for Claim $C$ in the proof of Proposition 3.18, one shows that $2 \alpha_{1}+2 \gamma$ cannot occur as a $T_{\text {ad }}$-weight in $\left(V / \mathfrak{g} \cdot x_{0}\right)^{G_{x_{0}}}$ when $m=2$. This proves Claim A.

We now proceed to Claim B. We fix $\beta=\alpha_{1}+\gamma$. We will show that the $T_{\text {ad }}$-weight $\beta$ has multiplicity at most one in $\left(V / \mathfrak{g} \cdot x_{0}\right)^{G_{x_{0}}}$. Since $T_{X_{0}} \mathrm{M}_{\mathcal{S}}^{G} \subseteq\left(V / \mathfrak{g} \cdot x_{0}\right)^{G_{x_{0}}}$, this implies Claim B. First off, we note that the $T_{\text {ad }}$-weight $\beta$ only occurs in $V\left(\lambda_{1}\right), V\left(\lambda_{3}\right)$ and in $V\left(\lambda_{4}\right)$, since $\beta$ belongs to the root lattice of $\operatorname{Sp}(2 n)$. Let $Z$ be the subspace of $V\left(\lambda_{1}\right) \oplus V\left(\lambda_{3}\right) \oplus$ $V\left(\lambda_{4}\right)$ consisting of $T_{\text {ad }}$-eigenvectors $v$ of $T_{\text {ad }}$-weight $\beta$ that satisfy the following three conditions:

$$
\begin{aligned}
& X_{\alpha_{1}} \cdot v \in\left\langle X_{-\left(\beta-\alpha_{1}\right)} x_{0}\right\rangle_{C} ; \\
& X_{\alpha_{2}} \cdot v \in\left\langle X_{-\left(\beta-\alpha_{2}\right)} x_{0}\right\rangle_{C} ; \text { and } \\
& X_{\alpha_{k}} \cdot v=0 \text { for all } k \in\{1,2, \ldots, n\} \backslash\{1,2\} .
\end{aligned}
$$

By Proposition 2.6, every $v \in V^{\beta}$ with $0 \neq[v] \in\left(V / \mathfrak{g} \cdot x_{0}\right)^{G_{x_{0}}}$ satisfies (3.56), (3.57) and (3.58). To show Claim $B$ it is therefore enough to prove that

$$
\operatorname{dim} Z \leq 2,
$$

since the nonzero vector $X_{-\beta} \cdot x_{0}$ belongs to $\mathfrak{g} \cdot x_{0} \cap Z$. The proof of (3.59) is the same as that of (3.42). 
3.15. (K23) The modules $\left(\left(\operatorname{Sp}(2 m) \times \mathbb{C}^{\times}\right) \times \operatorname{SL}(2) \times\left(\operatorname{Sp}(2 n) \times \mathbb{C}^{\times}\right),\left(\mathbb{C}^{2 m} \otimes \mathbb{C}^{2}\right) \oplus\left(\mathbb{C}^{2} \otimes\right.\right.$ $\left.\left.\mathbb{C}^{2 n}\right)\right)$ with $2 \leq m, n$. For these modules,

$$
\begin{aligned}
& E=\left\{\lambda_{1}, \lambda_{2}, \lambda_{3}, \lambda_{4}, \lambda_{5}, \lambda_{6}, \lambda_{7}\right\} ; \\
& d_{W}=5,
\end{aligned}
$$

where

$$
\begin{aligned}
& \lambda_{1}:=\omega_{1}+\varepsilon+\omega^{\prime} ; \quad \lambda_{2}:=\omega^{\prime}+\omega_{1}^{\prime \prime}+\varepsilon^{\prime} ; \quad \lambda_{3}:=\omega_{1}+\varepsilon+\omega_{1}^{\prime \prime}+\varepsilon^{\prime} ; \quad \lambda_{4}:=\omega_{2}+2 \varepsilon ; \\
& \lambda_{5}:=\omega_{2}^{\prime \prime}+2 \varepsilon^{\prime} ; \quad \lambda_{6}:=2 \varepsilon ; \quad \lambda_{7}:=2 \varepsilon^{\prime} .
\end{aligned}
$$

We remark that $G=\bar{G}$ is the only connected group between $\bar{G}^{\prime}$ and $\bar{G}$ for which these modules are spherical, cf. Remark 3.3. Therefore, we assume $G=\bar{G}=\left(\operatorname{Sp}(2 m) \times \mathbb{C}^{\times}\right) \times$ $\mathrm{SL}(2) \times\left(\operatorname{Sp}(2 n) \times \mathbb{C}^{\times}\right)$throughout this section.

In this section we will prove the following proposition.

Proposition 3.22. The $T_{\mathrm{ad}}$-module $T_{X_{0}} \mathrm{M}_{\mathcal{S}}^{G}$ is multiplicity-free. Its $T_{\mathrm{ad}}$-weight set is

$$
\left\{\alpha_{1}, \alpha_{1}+\gamma, \alpha^{\prime}, \alpha_{1}^{\prime \prime}, \alpha_{1}^{\prime \prime}+\gamma^{\prime \prime}\right\},
$$

where

$$
\begin{aligned}
\gamma & = \begin{cases}\alpha_{2} & \text { if } m=2 ; \\
2\left(\alpha_{2}+\alpha_{3}+\ldots+\alpha_{m-1}\right)+\alpha_{m} & \text { if } m>2 ;\end{cases} \\
\gamma^{\prime \prime} & = \begin{cases}\alpha_{2}^{\prime \prime} & \text { if } n=2 ; \\
2\left(\alpha_{2}^{\prime \prime}+\alpha_{3}^{\prime \prime}+\ldots+\alpha_{n-1}^{\prime \prime}\right)+\alpha_{n}^{\prime \prime} & \text { if } n>2 .\end{cases}
\end{aligned}
$$

In particular, $\operatorname{dim} T_{X_{0}} \mathrm{M}_{\mathcal{S}}^{G}=d_{W}$.

Proof. The argument is very similar to that of Propositions 3.8, 3.20 and 3.21. Let $\beta$ be a $T_{\text {ad }}$-weight in $T_{X_{0}} \mathrm{M}_{\mathcal{S}}^{G}$. It follows from Proposition 2.19 that at least one $\lambda \in E$ has a positive coefficient in the expression of $\beta$ as a $\mathbb{Z}$-linear combination of elements of $E$. Note that all elements of $E$ except $\lambda_{4}$ and $\lambda_{5}$ have codimension 1. In particular, if $\lambda \notin\left\{\lambda_{4}, \lambda_{5}\right\}$, then it follows from Proposition 2.21) that $\beta$ is a simple root belonging to the set (3.52), and that its weight space has dimension one.

Consequently, to prove the proposition, what remains is to show the following two claims:

Claim A: if $\lambda_{4}$ or $\lambda_{5}$ are the only element of $E$ which have a positive coefficient in the expression of $\beta$, then $\beta=\alpha_{1}+\gamma$ or $\beta=\alpha_{1}^{\prime \prime}+\gamma^{\prime \prime}$.

Claim B: the $T_{\text {ad }}$-weights $\beta=\alpha_{1}+\gamma$ and $\beta^{\prime \prime}=\alpha_{1}^{\prime \prime}+\gamma_{1}^{\prime \prime}$ have multiplicity at most one in $T_{X_{0}} \mathrm{M}_{\mathcal{S}}^{G}$.

We begin with Claim A. Recall from Proposition 2.6 that $\beta \in\langle E\rangle_{\mathbb{Z}} \cap\langle\Pi\rangle_{\mathbb{N}}$. Straightforward computations show that

$$
\left.\langle E\rangle_{\mathbb{Z}} \cap \Lambda_{R}=\underset{34}{\left\langle\alpha_{1}, \gamma\right.}, \alpha^{\prime}, \alpha_{1}^{\prime \prime}, \gamma^{\prime \prime}\right\rangle_{\mathbb{Z}}
$$


and that

$$
\begin{aligned}
\alpha_{1} & =\lambda_{1}+\lambda_{3}-\lambda_{2}-\lambda_{4} ; \\
\alpha^{\prime} & =\lambda_{1}+\lambda_{2}-\lambda_{3} ; \\
\alpha_{1}^{\prime \prime} & =\lambda_{2}+\lambda_{3}-\lambda_{1}-\lambda_{5} ; \\
\gamma & =2 \lambda_{4}+\lambda_{2}-\lambda_{1}-\lambda_{3}-\lambda_{6} ; \\
\gamma^{\prime \prime} & =2 \lambda_{5}+\lambda_{1}-\lambda_{2}-\lambda_{3}-\lambda_{7} .
\end{aligned}
$$

Let $K$ be the basis of $\langle E\rangle_{\mathbb{Z}} \cap \Lambda_{R}$ given in equation (3.61). Since $\beta \in\langle\Pi\rangle_{\mathbb{N}}$ and all elements of $K$ contain a simple root in their support, which is not in the support of any other element of $K$, it follows that $\beta \in\langle K\rangle_{\mathbb{N}}$. Therefore, there exist $A_{1}, A_{2}, A_{3}, A_{4}, A_{5} \in \mathbb{N}$ such that

$$
\beta=A_{1} \alpha_{1}+A_{2} \gamma+A_{3} \alpha^{\prime}+A_{4} \alpha_{1}^{\prime \prime}+A_{5} \gamma^{\prime \prime}
$$

From the hypothesis of Claim A, it follows that

$$
\left\{\begin{array}{l}
A_{1}-A_{2}+A_{3}-A_{4}+A_{5} \leq 0 \\
-A_{1}+A_{2}+A_{3}+A_{4}-A_{5} \leq 0 \\
A_{1}-A_{2}-A_{3}+A_{4}-A_{5} \leq 0
\end{array}\right.
$$

Adding the first two inequalities in (3.63) yields that $A_{3}=0$. Adding the first and the third inequality tells us that $A_{1} \leq A_{2}$, while adding the second and third gives $A_{4} \leq A_{5}$. Moreover, after substituting $A_{3}=0$, the first two inequalities also give us that $A_{2}-A_{1}=$ $A_{5}-A_{4}$. Put $C:=A_{2}-A_{1}=A_{5}-A_{4}$. Then $C \in \mathbb{N}$ and

$$
\begin{aligned}
\beta & =A_{1} \alpha_{1}+\left(A_{1}+C\right) \gamma+A_{4} \alpha_{1}^{\prime \prime}+\left(A_{4}+C\right) \gamma^{\prime \prime} \\
& =\left(A_{1}+2 C\right) \lambda_{4}+\left(A_{4}+2 C\right) \lambda_{5}-2 C \lambda_{3}-\left(A_{1}+C\right) \lambda_{6}-\left(A_{4}+C\right) \lambda_{7} .
\end{aligned}
$$

By Proposition 2.20, it follows from (3.65) that $A_{1}+A_{4}+4 C \leq 2$. This implies that $C=0$. The inequality $A_{1}+A_{4} \leq 2$ has five solutions in $\mathbb{N} \times \mathbb{N}$. This implies that $\beta \in\left\{\beta_{1}, \beta_{2}, \ldots, \beta_{5}\right\}$ where $\beta_{1}=\alpha_{1}+\gamma, \beta_{2}=\alpha_{1}^{\prime \prime}+\gamma^{\prime \prime}, \beta_{1}=\alpha_{1}+\gamma+\alpha_{1}^{\prime \prime}+\gamma^{\prime \prime}, \beta_{4}=$ $2 \alpha_{1}+2 \gamma$ and $\beta_{5}=2 \alpha_{1}^{\prime \prime}+2 \gamma^{\prime \prime}$. We cannot have $\beta=\beta_{3}$ because $\beta_{3}$ does not belong to $\Pi+\left(R^{+} \cup\{0\}\right)$. If $m>2$, then $\beta \neq \beta_{4}$ for the same reason. If $m=2$, then an argument like that for Claim $C$ in the proof of Proposition 3.18 shows that $\beta \neq \beta_{4}$. If $n>2$, then $\beta \neq \beta_{5}$ because $\beta_{5} \notin \Pi+\left(R^{+} \cup\{0\}\right)$. If $n=2$, then $\beta \neq \beta_{5}$ by an argument like that for Claim $C$ in the proof of Proposition 3.18. This proves Claim A.

The argument for Claim B is the same as that for Claim B in the proof of Proposition 3.20, except that one has to go through it twice: first for $\beta=\alpha_{1}+\gamma$ and then for $\beta^{\prime \prime}=\alpha_{1}^{\prime \prime}+\gamma^{\prime \prime}$. This finishes the proof.

3.16. (K24) The module $\left(\operatorname{Spin}(8) \times \mathbb{C}^{\times} \times \mathbb{C}^{\times}, \mathbb{C}_{+}^{8} \oplus \mathbb{C}_{-}^{8}\right)$. Here

$$
\begin{aligned}
& E=\left\{\omega_{3}+\varepsilon, \omega_{4}+\varepsilon^{\prime}, \omega_{1}+\varepsilon+\varepsilon^{\prime}, 2 \varepsilon, 2 \varepsilon^{\prime}\right\} ; \\
& d_{W}=3 .
\end{aligned}
$$

Proposition 3.23. The $T_{\mathrm{ad}}$-module $\left(V / \mathfrak{g} \cdot x_{0}\right)^{G_{x_{0}}^{\prime}}$ is multiplicity-free and has $T_{\mathrm{ad}}$-weight set

$$
\left\{\alpha_{1}+\alpha_{2}+\alpha_{3}, \alpha_{1}+\alpha_{2}+\alpha_{4}, \alpha_{2}+\alpha_{3}+\alpha_{4}\right\}
$$


In particular, $\operatorname{dim}\left(V / \mathfrak{g} \cdot x_{0}\right)^{G_{x_{0}}^{\prime}}=d_{W}$. Consequently, $\operatorname{dim} T_{X_{0}} \mathbf{M}_{\mathcal{S}}^{G}=d_{W}$

Proof. Note that $G^{\prime}=\operatorname{Spin}(8)$. Consider the $G^{\prime}$-module $V^{\prime}:=V\left(\omega_{1}\right) \oplus V\left(\omega_{3}\right) \oplus V\left(\omega_{4}\right)$ and its element $x_{0}^{\prime}=v_{\omega_{1}}+v_{\omega_{3}}+v_{\omega_{4}}$. Since $V(2 \varepsilon)$ and $V\left(2 \varepsilon^{\prime}\right)$ are subspaces of $\mathfrak{g} \cdot x_{0}$, we have that $\left(V^{\prime} / \mathfrak{g}^{\prime} \cdot x_{0}^{\prime}\right)^{G_{x_{0}}^{\prime}} \simeq\left(V / \mathfrak{g} \cdot x_{0}\right)^{G_{x_{0}}^{\prime}}$ as $T_{\text {ad }}$-modules. Observe that $G_{x_{0}}^{\prime}=G_{x_{0}^{\prime}}^{\prime}$.

The monoid $p\left(\langle E\rangle_{\mathbb{N}}\right)=\left\langle\omega_{1}, \omega_{3} \omega_{4}\right\rangle_{\mathbb{N}}$ is free and $G^{\prime}$-saturated. By [BCF08, Theorems 3.1 and 3.10], $\left(V^{\prime} / \mathfrak{g}^{\prime} \cdot x_{0}^{\prime}\right)^{G_{x_{0}}^{\prime}}$ is multiplicity-free and its $T_{\text {ad }}$-weights belong to Table 1 in [BCF08, page 2810]. By Proposition 2.6, the $T_{\text {ad }}$-weights of $\left(V^{\prime} / \mathfrak{g}^{\prime} \cdot x_{0}^{\prime}\right)^{G_{x_{0}}^{\prime}}$ also belong to $\Lambda_{R} \cap\left\langle\omega_{1}, \omega_{3}, \omega_{4}\right\rangle_{\mathbb{Z}}$. A straightforward computation shows that

$$
\Lambda_{R} \cap\left\langle\omega_{1}, \omega_{3}, \omega_{4}\right\rangle_{\mathbb{Z}}=\left\{a \alpha_{1}+b \alpha_{2}+c \alpha_{3}+d \alpha_{4} \mid a, b, c, d \in \mathbb{Z} \text { and } 2 b=a+c+d\right\} .
$$

Let $\gamma$ be a $T_{\text {ad }}$-weight of $\left(V^{\prime} / \mathfrak{g}^{\prime} \cdot x_{0}^{\prime}\right)^{G_{x_{0}}^{\prime}}$. It follows from equation (3.66) that

$$
|\operatorname{supp}(\gamma)| \geq 2 \text { and } \alpha_{2} \in \operatorname{supp}(\gamma) \text {. }
$$

There are twelve $T_{\text {ad }}$-weights in [BCF08, Table 1, page 2810] that satisfy (3.67). Six of them are

$$
\alpha_{1}+\alpha_{2}, \alpha_{2}+\alpha_{3}, \alpha_{2}+\alpha_{4}, \alpha_{1}+2 \alpha_{2}+\alpha_{3}, \alpha_{1}+2 \alpha_{2}+\alpha_{4}, 2 \alpha_{2}+\alpha_{3}+\alpha_{4}
$$

but $\gamma$ cannot be among these since they do not belong to $\Lambda_{R} \cap\left\langle\omega_{1}, \omega_{3}, \omega_{4}\right\rangle_{\mathbb{Z}}$ by equation (3.66).

Three more $T_{\text {ad }}$-weights in [BCF08, Table 1] that satisfy (3.67) are

$$
\begin{aligned}
& \gamma_{1}:=2 \alpha_{1}+2 \alpha_{2}+\alpha_{3}+\alpha_{4}=2 \omega_{1} \\
& \gamma_{3}:=\alpha_{1}+2 \alpha_{2}+2 \alpha_{3}+\alpha_{4}=2 \omega_{3} \\
& \gamma_{4}:=\alpha_{1}+2 \alpha_{2}+\alpha_{3}+2 \alpha_{4}=2 \omega_{4}
\end{aligned}
$$

We claim that none of them is a $T_{\text {ad }}$-weight in $\left(V^{\prime} / \mathfrak{g}^{\prime} \cdot x_{0}^{\prime}\right)^{G_{x_{0}}^{\prime}}$. Let $i \in\{1,3,4\}$. We will argue by contradiction that $\gamma_{i}$ is not a $T_{\text {ad }}$-weight in $\left(V^{\prime} / \mathfrak{g}^{\prime} \cdot x_{0}^{\prime}\right)^{G_{x_{0}}^{\prime}}$. Assume $v \in V^{\prime}$ is a $T_{\text {ad }}$-eigenvector of weight $\gamma_{i}$ such that $[v]$ is nonzero in $\left(V^{\prime} / \mathfrak{g}^{\prime} \cdot x_{0}^{\prime}\right)^{G_{x_{0}}^{\prime}}$. Note that $\alpha_{i}$ is the only simple root $\beta$ such that $\gamma_{i}-\beta$ is in $R^{+} \cup\{0\}$. By Proposition 2.6 this implies that

$$
X_{\alpha_{i}} \cdot v \in\left\langle X_{-\left(\gamma_{i}-\alpha_{i}\right)} x_{0}^{\prime}\right\rangle_{\mathbb{C}} \backslash\{0\} .
$$

Because $\left\langle\left(\gamma_{i}-\alpha_{i}\right)^{\vee}, \cdot\right\rangle$ is nonzero on $\omega_{1}, \omega_{3}$ and $\omega_{4}$, we have that $X_{-\left(\gamma_{i}-\alpha_{i}\right)} x_{0}^{\prime}$ has nonzero projection on the three summands $V\left(\omega_{1}\right), V\left(\omega_{3}\right)$ and $V\left(\omega_{4}\right)$ of $V^{\prime}$. On the other hand, one checks with $L i E$ that $\gamma_{i}$ does not occur as a $T_{\text {ad }}$-weight in all three components of $V^{\prime}$ (see the proof of Proposition 3.15 for the code of a similar compuation in LiE). This contradiction with equation (3.68) proves the claim.

The remaining three $T_{\mathrm{ad}}$-weights in [BCF08, Table 1] that satisfy equation (3.67) are the three weights listed in the proposition. Since $d_{W}=3$ this proves the proposition.

\section{APPENDIX: COMPUTING THE INVARIANTS OF THE MODULES IN THE FAMILY K5}

During the work for the present paper, we also developed a different technique which explicitly computes the invariants in $\left(V / \mathfrak{g} \cdot x_{0}\right)^{G_{x_{0}}}$. The main idea is to use theoretical and elementary arguments to reduce the problem to the study of the smallest case for the parameter $n$ (or the parameters $(n, m)$ ), and then do a direct computation for the smallest 
case. In this appendix we present the method for the K5 family. The method works equally well for the study of the remaining infinite families.

3.17. Notation and Generalities about $\mathrm{Sp}_{2 n}$. To accommodate the computational and explicit nature of this appendix, the notation used here is different from that used in the rest of the paper. Assume $n \geq 1$ is a positive integer. Consider the vector space $\mathbb{C}^{2 n}$ with basis $e_{1}, \ldots, e_{2 n}$. We also set $f_{i}=e_{2 n+1-i}$ for $1 \leq i \leq n$.

We define a nondegenerate skewsymmetric bilinear form $\Omega: \mathbb{C}^{2 n} \times \mathbb{C}^{2 n} \rightarrow \mathbb{C}$ by

$$
\Omega\left(e_{i}, e_{j}\right)=\Omega\left(f_{i}, f_{j}\right)=0, \quad \Omega\left(e_{i}, f_{j}\right)=\delta_{i j}, \quad \Omega\left(f_{i}, e_{j}\right)=-\delta_{i j}
$$

for $1 \leq i, j \leq n$, where $\delta_{i j}$ denotes the Kronecker delta function. By definition $\mathrm{Sp}_{2 n}$ consists of the linear automorphisms $g$ of $\mathbb{C}^{2 n}$ which have the property $\Omega(g(v), g(w))=\Omega(v, w)$ for all $v, w \in \mathbb{C}^{2 n}$.

We denote by $\mathfrak{s p}_{2 n}$ the Lie algebra of $\mathrm{Sp}_{2 n}$. According to [GW09, p.72, Eq (2.8)] it has a basis

$$
\left\{a_{i j}, b_{k l}, c_{k l}: 1 \leq i, j \leq n, 1 \leq k \leq l \leq n\right\}
$$

defined as follows: $a_{i j}=\left(e_{j} \mapsto e_{i}, f_{i} \mapsto-f_{j}\right)$ where the notation means that $a_{i j}\left(e_{j}\right)=$ $e_{i}, a_{i j}\left(f_{i}\right)=-f_{j}, a_{i j}\left(e_{t}\right)=0$ if $1 \leq t \leq n$ and $t \neq j$, and $a_{i j}\left(f_{t}\right)=0$ if $1 \leq t \leq n$ and $t \neq i$. With the same notational convention $b_{k l}=\left(e_{l} \mapsto f_{k}, e_{k} \mapsto f_{l}\right)$ and $c_{k l}=\left(f_{l} \mapsto e_{k}, f_{k} \mapsto e_{l}\right)$.

3.18. Notation for the $\mathrm{K} 5$ example. By definition, for $n \geq 2, \mathrm{~K} 5$ with parameter $n$, or more simply $\mathrm{K} 5(n)$, is the $\mathrm{K} 5$ family in List (3.1) with group $\mathrm{Sp}_{2 n} \times \mathrm{GL}_{2}$ and $W=\mathbb{C}^{2 n} \otimes \mathbb{C}^{2}$. Fix $n \geq 2$. Set $G=\mathrm{Sp}_{2 n} \times \mathrm{GL}_{2}$.

We denote by $\varepsilon_{1}, \ldots, \varepsilon_{n}$ the standard basis of the weight lattice of $\operatorname{Sp}_{2 n}$ and by $\varepsilon_{1}^{\prime}, \varepsilon_{2}^{\prime}$ the standard basis of the weight lattice of $\mathrm{GL}_{2}$. For $1 \leq i \leq n$ we denote by $\omega_{i}$ the $i$-th fundamental weight of $\mathrm{Sp}_{2 n}$, and for $1 \leq i \leq 2$ we denote by $\omega_{i}^{\prime}$ the $i$-th fundamental weight of $\mathrm{GL}_{2}$. We set:

$$
V_{1}^{\prime}=V\left(\omega_{1}\right) \otimes V\left(\omega_{1}^{\prime}\right), \quad V_{2}^{\prime}=\wedge^{2} \mathbb{C}^{2 n} \otimes V\left(\omega_{2}^{\prime}\right), \quad V_{3}^{\prime}=V\left(\omega_{2}^{\prime}\right), \quad V^{\prime}=\oplus_{i=1}^{3} V_{i}^{\prime} .
$$

We also set

$$
V_{2}=V\left(\omega_{2}\right) \otimes V\left(\omega_{2}^{\prime}\right) \subset V_{2}^{\prime}, \quad V_{i}=V_{i}^{\prime} \text { for } i \neq 2, \text { and } V=\oplus_{i=1}^{3} V_{i} .
$$

We define, for $1 \leq i \leq 3$, dominant weights $\lambda_{i}$ of $G$ by $V_{i}=V\left(\lambda_{i}\right)$. Hence $\lambda_{1}=\omega_{1}+\omega_{1}^{\prime}$, $\lambda_{2}=\omega_{2}+\omega_{2}^{\prime}, \lambda_{3}=\omega_{2}^{\prime}$. We denote by $T$ the diagonal maximal torus of $G$. We define an action $\rho^{\prime}: T \times V^{\prime} \rightarrow V^{\prime}$ by $\rho^{\prime}\left(t, \sum_{i=1}^{3} w_{i}\right)=\sum_{i=1}^{3} \lambda_{i}(t) t^{-1} \cdot w_{i}$, for $t \in T$ and $w_{i} \in V_{i}^{\prime}$ for $1 \leq i \leq 3$. It is clear that for $t \in T$ and $w \in V$ we have $\rho^{\prime}(t, w)=\alpha(t, w)$, where $\alpha: T \times V \rightarrow V$ is the action defined in [PVS12, Definition 2.11].

For the $\mathbb{C}^{2 n}$ that $S p_{2 n}$ acts we fix a basis $e_{1}, \ldots, e_{2 n}$ and define $\Omega, f_{i}, a_{i j}, b_{k l}, c_{k l}$ as in Subsection 3.17. For the $\mathbb{C}^{2}$ that $\mathrm{GL}_{2}$ acts we fix a basis $g_{1}, g_{2}$ and define a basis $\left\{d_{p q}: 1 \leq\right.$ $p, q \leq 2\}$ of $\mathfrak{g l}_{2}$ by $d_{p q}\left(e_{a}\right)=e_{p}$ if $q=a$ and 0 otherwise. Then the set

$$
\left\{a_{i j}, b_{k l}, c_{k l}, d_{p q}: 1 \leq i, j \leq n, 1 \leq k \leq l \leq n, 1 \leq p, q \leq 2\right\}
$$

is a basis of $\mathfrak{g}$ which we call the standard basis.

We set $\mathcal{A}=\{1,2,2 n-1,2 n\}$ and $H=\sum_{i=1}^{n} e_{i} \wedge f_{i} \in \wedge^{2} \mathbb{C}^{2 n}, \quad H_{s}=\sum_{i=1}^{2} e_{i} \wedge f_{i} \in$ $\wedge^{2} \mathbb{C}^{2 n}, \quad x_{0}=e_{1} \otimes g_{1}+e_{1} \wedge e_{2} \otimes g_{1} \wedge g_{2}+g_{1} \wedge g_{2}$. For $1 \leq i \leq 3$ we denote by $v_{i}$ the component of $x_{0}$ that is in $V_{i}$. For example, $v_{3}=g_{1} \wedge g_{2}$. 
We say that $v \in V^{\prime}$ is an invariant if $[v] \in\left(V^{\prime} / \mathfrak{g} \cdot x_{0}\right)^{G_{x_{0}}}$. For $1 \leq i \leq 2 n$ we define that the index of $e_{i}$ is $i$. Since $f_{i}=e_{2 n+1-i}$, we also define that the index of $f_{i}$ is $2 n+1-i$. We denote by $X_{0} \subset V$ the Zariski closure of the G-orbit of $x_{0}$.

We fix the following basis of $V^{\prime}$ which we call the monomial basis:

$$
e_{i} \otimes g_{p}: 1 \leq i \leq 2 n, 1 \leq p \leq 2, \quad e_{i} \wedge e_{j} \otimes g_{1} \wedge g_{2}: 1 \leq i<j \leq 2 n, \quad g_{1} \wedge g_{2} .
$$

For $v \in V^{\prime}$, the terms of $v$ are by definition the nonzero monomial terms of the (unique) expression of $v$ as a linear combination of the monomial basis. For example, the terms of $2 e_{2} \otimes g_{1}+7 g_{1} \wedge g_{2}$ are $2 e_{2} \otimes g_{1}$ and $7 g_{1} \wedge g_{2}$.

We denote by $V_{a}^{\prime}$, the linear span of the subset of the monomial basis of $V^{\prime}$ where all indices appearing for $e_{i}$ are in $\mathcal{A}$. In more detail, $V_{a}^{\prime}$ is the linear span of

$e_{i} \otimes g_{p}: i \in \mathcal{A}, 1 \leq p \leq 2, \quad e_{i} \wedge e_{j} \otimes g_{1} \wedge g_{2}: 1 \leq i<j \leq 2 n,\{i, j\} \subset \mathcal{A}, \quad g_{1} \wedge g_{2}$.

We denote by $V_{b}^{\prime}$ the linear span of the remaining elements of the monomial basis of $V^{\prime}$.

We define a Lie subalgebra $Z_{a} \subset \mathfrak{g}$ and a vector subspace $Z_{b} \subset \mathfrak{g}$ such that, as vector space, $\mathfrak{g}$ is the direct sum of $Z_{a}$ and $Z_{b}$. Namely, we set $Z_{a}$ to be the linear span of

$$
\left\{a_{i j}, b_{k l}, c_{k l}, d_{i j}: \quad 1 \leq i, j \leq 2,1 \leq k \leq l \leq 2\right\}
$$

and $Z_{b}$ to be the linear span of the remaining elements of the standard basis of $\mathfrak{g}$. Clearly $Z_{a}$ is in a natural way isomorphic to $\mathfrak{s p}_{4} \oplus \mathfrak{g l}_{2}$ which is the Lie algebra of the group $\mathrm{Sp}_{4} \times$ $\mathrm{GL}_{2}$ of the case $\mathrm{K} 5(2)$.

An easy direct computation proves the following proposition.

Proposition 3.24. We have $Z_{a} \cdot V_{a}^{\prime} \subset V_{a}^{\prime}, Z_{a} \cdot V_{b}^{\prime} \subset V_{b}^{\prime}$ and $Z_{b} \cdot V_{a}^{\prime} \subset V_{b}^{\prime}$. As a corollary, $Z_{a} \cdot x_{0} \subset V_{a}^{\prime}$ and $Z_{b} \cdot x_{0} \subset V_{b}^{\prime}$.

Corollary 3.25. Assume $v \in \mathfrak{g} \cdot x_{0}$. Write $v=v_{a}+v_{b}$ with $v_{a} \in V_{a}^{\prime}, v_{b} \in V_{b}^{\prime}$. Then there exist $z_{a} \in Z_{a}$ and $z_{b} \in Z_{b}$ with $v_{a}=z_{a} \cdot x_{0}$ and $v_{b}=z_{b} \cdot x_{0}$.

Proof. There exists $z \in \mathfrak{g}$ with $v=z \cdot x_{0}$. Write $z=z_{a}+z_{b}$ with $z_{a} \in Z_{a}$ and $z_{b} \in Z_{b}$, then $v=z_{a} \cdot x_{0}+z_{b} \cdot x_{0}$. Using Proposition 3.24 $z_{a} \cdot x_{0} \in V_{a}^{\prime}$ and $z_{b} \cdot x_{0} \in V_{b}^{\prime}$. Since $V_{a}^{\prime} \cap V_{b}^{\prime}=\{0\}$ we get $v_{a}=z_{a} \cdot x_{0}$ and $v_{b}=z_{b} \cdot x_{0}$.

We denote by $\mathfrak{g}_{x_{0}}$ the Lie algebra of the stabilizer $G_{x_{0}}$ of the point $x_{0}$. It will be computed in Proposition 3.28 .

Corollary 3.26. Assume $v \in V^{\prime}$ is an invariant. Write $v=v_{a}+v_{b}$ with $v_{a} \in V_{a}^{\prime}$ and $v_{b} \in V_{b}^{\prime}$. Set $F=Z_{a} \cap \mathfrak{g}_{x_{0}}$. Then $F \cdot v_{a} \subset Z_{a} \cdot x_{0}$.

Proof. If $n=2$ then $v=v_{a}$ and the result is obvious. Assume $n \geq 3$ and let $z \in F$. Since $F \subset \mathfrak{g}_{x_{0}}$ and $v$ is an invariant we have $z \cdot v \in \mathfrak{g} \cdot x_{0}$. Hence $z \cdot v_{a}+z \cdot v_{b} \in \mathfrak{g} \cdot x_{0}$. Since $z \in Z_{a}$, by Proposition $3.24 z \cdot v_{a} \in V_{a}^{\prime}$ and $z \cdot v_{b} \in V_{b}^{\prime}$. Hence $z \cdot v_{a}+z \cdot v_{b}$ is the decomposition of $z \cdot v$ with components in $V_{a}^{\prime}$ and $V_{b}^{\prime}$. Using Corollary 3.25 we have that $z \cdot v_{a} \in Z_{a} \cdot x_{0}$.

Direct computations give the following two propositions.

Proposition 3.27. The stabilizer subgroup $G_{x_{0}}$ is equal to the set of $\left(h_{1}, h_{2}\right) \in \mathrm{Sp}_{2 n} \times \mathrm{GL}_{2}$ which have the property that there exists $b \in \mathbb{C}^{*}$ and $a_{1}, a_{2} \in \mathbb{C}$ such that

$$
h_{1}\left(e_{1}\right)=b e_{1}, \quad h_{1}\left(e_{2}\right)=b^{-1} e_{2}+a_{1} e_{1}, \quad h_{2}\left(g_{1}\right)=b^{-1} g_{1}, \quad h_{2}\left(g_{2}\right)=b g_{2}+a_{2} g_{1} .
$$


Proposition 3.28. The following set

$$
\begin{gathered}
\left\{a_{12}\right\} \cup\left\{a_{i j}: 1 \leq i \leq n, 3 \leq j \leq n\right\} \cup\left\{b_{i j}: 3 \leq i \leq j \leq n\right\} \cup \\
\left\{c_{i j}: 1 \leq i \leq j \leq n\right\} \cup\left\{d_{12}\right\} \cup\left\{d_{11}-a_{11}-d_{22}+a_{22}\right\}
\end{gathered}
$$

is a basis for $\mathfrak{g}_{x_{0}}$.

Lemma 3.29. Assume $v \in V^{\prime}$ is an invariant for K5(n). Write $v=v_{a}+v_{b}$ with $v_{a} \in V_{a}^{\prime}$ and $v_{b} \in V_{b}^{\prime}$. Then $\left[v_{a}\right] \in\left(V^{\prime} / \mathfrak{g} \cdot x_{0}\right)^{\mathfrak{g}_{0}}$ for K5(2).

Proof. Using Proposition 3.28, which implies compatibility of $\mathfrak{g}_{x_{0}}$ for $\mathrm{K} 5(n)$ as $n$ varies, the result follows by Corollary 3.26 .

Proposition 3.30. Assume $v \in V^{\prime}$ is a $\rho^{\prime}$-weight vector such that $[v] \in\left(V^{\prime} / \mathfrak{g} \cdot x_{0}\right)^{G_{x_{0}}}$ and $[v] \neq 0$. Denote by $\beta$ the $\rho^{\prime}$-weight of $v$. Then $\beta$ is a linear combination with integer coefficients of the $\lambda_{i}$.

Proof. Write $v=w_{1}+w_{2}+w_{3}$ with $w_{j} \in V_{j}^{\prime}$. Since $v \neq 0$ there exists $i$ with $1 \leq i \leq 3$ such that $w_{i} \neq 0$. Then the arguments in the proof of [PVS12, Lemma 2.17(c)] also work here, taking into account that by the definition of $\rho^{\prime}$ we have that $w_{i}$ is a $T$-weight vector with $T$-weight equal to $\lambda_{i}-\beta$.

3.19. Analysis of the invariants of $K 5$ with a high index. Assume $n \geq 2$ and we are in $\mathrm{K} 5(n)$ case. Proposition 3.34 will give a strong restriction on the invariants $v$ with $v \notin V_{a}^{\prime}$. Recall $H=\sum_{i=1}^{n} e_{i} \wedge f_{i}$. We set $\gamma_{2}=\varepsilon_{1}+\varepsilon_{2}$ and $q^{(2)}=H \otimes g_{1} \wedge g_{2}$.

Remark 3.31. We have computed $G_{x_{0}}$ in Proposition 3.27. A small computation shows that $q^{(2)}$ is an invariant.

Lemma 3.32. Assume $v \in V^{\prime}$ such that $0 \neq[v] \in\left(V^{\prime} / \mathfrak{g} \cdot x_{0}\right)^{G_{x_{0}}}$. Assume $v$ is a $\rho^{\prime}$-weight vector. Denote by $\beta$ the $\rho^{\prime}$-weight of $v$. Assume $w$ is an element of the monomial basis of $V^{\prime}$ such that $w \in V_{b}^{\prime}$ and a nonzero multiple of $w$ is a term of $v$. Then $n \geq 3, \beta=\gamma_{2}, w \in V_{2}^{\prime}$ and there exists $i$ with $3 \leq i \leq n$ such that $w=e_{i} \wedge f_{i} \otimes g_{1} \wedge g_{2}$.

Proof. Denote by $\Gamma$ the $\mathbb{Z}$-span of the weights $\varepsilon_{1}, \varepsilon_{2}, \varepsilon_{1}^{\prime}, \varepsilon_{2}^{\prime}$. We will use that by Proposition $3.30 \beta \in \Gamma$. The assumption $w \in V_{b}^{\prime}$ implies that there exists $j$ with $3 \leq j \leq 2 n-2$ such that $e_{j}$ appears in $w$. If $w \in V_{1}^{\prime}$ then $w=e_{j} \otimes g_{p}$ with $1 \leq p \leq 2$ which implies that $\beta \notin \Gamma$, a contradiction. Hence $w \in V_{2}^{\prime}$, so $w=e_{p} \wedge e_{q} \otimes g_{1} \wedge g_{2}$ for some $1 \leq p<q \leq n$, or $w=e_{p} \wedge f_{q} \otimes g_{1} \wedge g_{2}$ for some $1 \leq p, q \leq n$, or $w=f_{p} \wedge f_{q} \otimes g_{1} \wedge g_{2}$ for some $1 \leq p<q \leq n$. The first and the third cases are impossible, since then $\beta \notin \Gamma$. The second case is possible if and only if $p=q$.

We need the following lemma, which restricts further the candidate invariants.

Lemma 3.33. Assume $c_{t} \in \mathbb{C}$, for $1 \leq t \leq n$. Set $z=\left(\sum_{t=1}^{n} c_{t} e_{t} \wedge f_{t}\right) \otimes g_{1} \wedge g_{2}$. Assume $v \in V^{\prime}$ is an invariant which is also a $\rho^{\prime}$-weight vector with $\rho^{\prime}$-weight $\gamma_{2}$ such that, for all $1 \leq t \leq n$ and $d \in \mathbb{C}^{*}$, we have that $d e_{t} \wedge f_{t} \otimes g_{1} \wedge g_{2}$ is not a term of $v-z$. Then $c_{i}=c_{j}$ for all $3 \leq i<j \leq n$.

Proof. Fix $i, j$ with $3 \leq i<j \leq n$. We assume $c_{i} \neq c_{j}$ and we will get a contradiction. Set $w=e_{i} \wedge f_{j} \otimes g_{1} \wedge g_{2}$. 
We act by the element $a_{i j}=\left(e_{j} \mapsto e_{i}, f_{i} \mapsto-f_{j}\right)$ which, by Proposition 3.28, is in $\mathfrak{g}_{x_{0}}$. We have $a_{i j} \cdot z=\left(c_{i}-c_{j}\right) w$. Lemma 3.32, which restricts the terms that can appear in $v$, implies that no nonzero muliple of $w$ can appear as a term of $a_{i j} \cdot(v-z)$. Hence $\left(c_{i}-c_{j}\right) w$ appears as a term of $a_{i j} \cdot v$. Since $v$ is an invariant, we get that $a_{i j} \cdot v \in \mathfrak{g} \cdot x_{0}$, hence there exists $z \in \mathfrak{g}$ such that $\left(c_{i}-c_{j}\right) w$ is a term of $z \cdot x_{0}$. Since $x_{0}$ has all indices less or equal to 2 it follows that each term of $z \cdot x_{0}$ can have at most one index $\geq 3$. This is a contradiction, since $w$ has two distinct indices, namely $i, 2 n+1-j$, greater or equal than 3 .

The following proposition is an important step for the reduction of the problem of invariants for the case $\mathrm{K} 5(n)$ to the case $\mathrm{K} 5(2)$.

Proposition 3.34. Assume $v \in V^{\prime}$ such that $0 \neq[v] \in\left(V^{\prime} / \mathfrak{g} \cdot x_{0}\right)^{G_{x_{0}}}$. Assume $v$ is a $\rho^{\prime}$-weight vector. Denote by $\beta$ the $\rho^{\prime}$-weight of $v$. Write $v=v_{a}+v_{b}$ with $v_{a} \in V_{a}^{\prime}$ and $v_{b} \in V_{b}^{\prime}$. Assume $v_{b} \neq 0$. Then $\beta=\gamma_{2}$ and there exists $c \in \mathbb{C}^{*}$ such that $v-c q^{(2)}$ is an invariant contained in $V_{a}^{\prime}$.

Proof. Lemma 3.32 implies that $\beta=\gamma_{2}$. Combining Lemmas 3.32 and 3.33 it follows that there exist $c \in \mathbb{C}^{*}$ such that $v-c q^{(2)} \in V_{a}^{\prime}$. By Remark $3.31 q^{(2)}$ is an invariant, hence $v-c q^{(2)}$ is an invariant contained in $V_{a}^{\prime}$.

3.20. The invariants of K5. Recall $H_{s}=\sum_{i=1}^{2} e_{i} \wedge f_{i}$ and $\gamma_{2}=\varepsilon_{1}+\varepsilon_{2}$. We define the following $\rho^{\prime}$-weights and weight vectors in $V^{\prime}$ : We set $\gamma_{1}=\varepsilon_{1}-\varepsilon_{2}+\varepsilon_{1}^{\prime}-\varepsilon_{2}^{\prime}$, and $r_{1}=$ $e_{2} \otimes g_{2}$. We set $r_{2,1}=H_{s} \otimes g_{1} \wedge g_{2}$ and $r_{2,2}=e_{1} \wedge f_{1} \otimes g_{1} \wedge g_{2}$. Then $r_{1}$ is a $\rho^{\prime}$-weight vector with $\rho^{\prime}$-weight $\gamma_{1}$ and $r_{2,1}$ and $r_{2,2}$ are $\rho^{\prime}$-weight vectors with $\rho^{\prime}$-weight $\gamma_{2}$.

An easy direct computation gives the following proposition.

Proposition 3.35. Assume we are in K5(2). Then $\left(V^{\prime} / \mathfrak{g} \cdot x_{0}\right)^{G_{x_{0}}}=\left(V^{\prime} / \mathfrak{g} \cdot x_{0}\right)^{\mathfrak{g}_{0}}$ and the classes in $V^{\prime} / \mathfrak{g} \cdot x_{0}$ of the elements

is a basis for the vector space $\left(V^{\prime} / \mathfrak{g} \cdot x_{0}\right)^{G_{x_{0}}}$.

$$
r_{1}, r_{2,1}, r_{2,2}
$$

Proposition 3.36. Assume $n \geq 2$ and we are in $K 5(n)$. Then the classes in $V^{\prime} / \mathfrak{g} \cdot x_{0}$ of the elements

is a basis for the vector space $\left(V^{\prime} / \mathfrak{g} \cdot x_{0}\right)^{G_{x_{0}}}$.

$$
r_{1}, q^{(2)}, r_{2,2}
$$

Proof. If $n=2$ then the result follows from Proposition 3.35, So we assume that $n \geq 3$.

We have computed $G_{x_{0}}$ in Proposition 3.27. A small calculation shows that the vectors in the statement of the present proposition are indeed invariants for $\mathrm{K} 5(n)$.

We will use that if an invariant $v$ for $\mathrm{K} 5(n)$ is an element of $V_{a}^{\prime}$ then, by Lemma 3.29, $[v] \in\left(V^{\prime} / \mathfrak{g} \cdot x_{0}\right)^{\mathfrak{g}_{0}}$ for $\mathrm{K} 5(2)$, hence by Proposition 3.35 $v$ is an invariant for K5(2).

Assume $v \in V^{\prime}$ is an invariant which is also a $\rho^{\prime}$-weight vector with $\rho^{\prime}$-weight $\beta$. Write $v=v_{a}+v_{b}$ with $v_{a} \in V_{a}^{\prime}$ and $v_{b} \in V_{b}^{\prime}$. We will show that $[v]$ is in the linear subspace of $V^{\prime} / \mathfrak{g} \cdot x_{0}$ spanned by the classes of the elements in the statement of the present proposition. Since these classes are linearly independent it will then follow that the classes are a basis for $\left(V^{\prime} / \mathfrak{g} \cdot x_{0}\right)^{G_{x_{0}}}$.

We first prove that there exists $i$ with $1 \leq i \leq 2$ such that $\beta=\gamma_{i}$. If $v_{b} \neq 0$ it follows from Proposition 3.34 that $\beta=\gamma_{2}$. If $v_{b}=0$ then $v$ is an invariant for K5(2), hence the existence of $i$ such that $\beta=\gamma_{i}$ follows from Proposition 3.35. 
Assume $\beta=\gamma_{1}$. By Proposition $3.34 v_{b}=0$ and hence $v$ is an invariant for the case K5(2). By Proposition 3.35 the vector subspace of $\left(V^{\prime} / \mathfrak{g} \cdot x_{0}\right)^{G_{x_{0}}}$ consisting of vectors with $\rho^{\prime}$-weight $\gamma_{1}$ is 1-dimensional spanned by $\left[r_{1}\right]$. Hence $[v]$ is in the linear span of $\left[r_{1}\right]$.

Assume $\beta=\gamma_{2}$. It follows from Proposition 3.34 that there exists $c_{1} \in \mathbb{C}$ such that $v-c_{1} q^{(2)}$ is an invariant contained in $V_{a}^{\prime}$. Consequently, by Proposition 3.35 there exist $c_{2}, c_{3} \in \mathbb{C}$ with

$$
v-c_{1} q^{(2)}-\left(c_{2} r_{2,1}+c_{3} r_{2,2}\right) \in \mathfrak{g} \cdot x_{0} .
$$

We will show $c_{2}=0$. Assume $c_{2} \neq 0$ and we will get a contradiction. We act by the element $a_{23}=\left(e_{3} \mapsto e_{2}, f_{2} \mapsto-f_{3}\right) \in \mathfrak{g}_{x_{0}}$. Since for all $b \in \mathfrak{g}_{x_{0}}$ we have $b \cdot\left(\mathfrak{g} \cdot x_{0}\right) \subset\left(\mathfrak{g} \cdot x_{0}\right)$, we get $a_{23} \cdot\left(\mathfrak{g} \cdot x_{0}\right) \subset \mathfrak{g} \cdot x_{0}$. Since $v, q^{(2)}$ are invariants, we have that $a_{23} \cdot\left(v-c_{1} q^{(2)}\right) \in$ $\mathfrak{g} \cdot x_{0}$. Hence, Equation (3.69) implies $a_{23} \cdot\left(c_{2} r_{2,1}+c_{3} r_{2,2}\right) \in \mathfrak{g} \cdot x_{0}$. Since $r_{2,2}$ is an invariant and $c_{2} \neq 0$ it follows that $a_{23} \cdot r_{2,1} \in \mathfrak{g} \cdot x_{0}$, hence $e_{2} \wedge f_{3} \otimes g_{1} \wedge g_{2} \in \mathfrak{g} \cdot x_{0}$. This is a contradiction, since by Lemma 2.17 (for $t=1$ ) the set of nonzero elements of $\mathfrak{g} \cdot x_{0}$ with $\rho^{\prime}$-weight $\varepsilon_{2}+\varepsilon_{3}$ is equal to $\left\{d\left(b_{13} \cdot x_{0}\right): d \in \mathbb{C}^{*}\right\}=\left\{d\left(f_{3} \otimes g_{1}-e_{2} \wedge f_{3} \otimes g_{1} \wedge g_{2}\right): d \in\right.$ $\left.\mathbb{C}^{*}\right\}$.

Proposition 3.37. Assume $n \geq 2$ and we are in $K 5(n)$. Then the classes in $V / \mathfrak{g} \cdot x_{0}$ of the following elements of $V$

is a basis for the vector space $\left(V / \mathfrak{g} \cdot x_{0}\right)^{r_{1}, \quad n r_{2,2}-q^{(2)}}$

Proof. By [FH91, Theorem 17.5] $V\left(\omega_{2}\right) \subset \wedge^{2} \mathbb{C}^{2 n}$ is the kernel of the linear map $\wedge^{2} \mathbb{C}^{2 n} \rightarrow \mathbb{C}$ uniquely specified by $u_{1} \wedge u_{2} \mapsto \Omega\left(u_{1}, u_{2}\right)$ for all $u_{1}, u_{2} \in \mathbb{C}^{2 n}$. Hence a basis of $V\left(\omega_{2}\right)$ is

$$
e_{i} \wedge e_{j}, \quad f_{i} \wedge f_{j}, \quad e_{a} \wedge f_{b}, \quad e_{k} \wedge f_{k}-e_{1} \wedge f_{1}
$$

with indices $1 \leq i<j \leq n, 2 \leq k \leq n, 1 \leq a, b \leq n$ with $a \neq b$.

Using Proposition 3.36, the result follows by an easy computation.

Proposition 3.38. Assume $n \geq 2$ and we are in $K 5(n)$. Then the vector subspace of $(V / \mathfrak{g}$. $\left.x_{0}\right)^{G_{x_{0}}}$ consisting of the vectors with the property that the section in $H^{0}\left(G \cdot x_{0}, \mathcal{N}_{X_{0}}\right)^{G}$ they induce extends to $X_{0}$ is equal to $\left(V / \mathfrak{g} \cdot x_{0}\right)^{G_{x_{0}}}$.

Proof. It is enough to show that for each of the 2 basis elements in the statement of Proposition 3.37 the induced section in $H^{0}\left(G \cdot x_{0}, \mathcal{N}_{X_{0}}\right)^{G}$ extends to $X_{0}$. Recall $x_{0}=\sum_{i=1}^{3} v_{i}$ with $v_{i} \in V_{i}$. For $1 \leq i \leq 3$ we set $w_{i}=x_{0}-v_{i}$. Using [PVS12, Proposition 3.1] we have that, for $i=1,2$, the codimension in $X_{0}$ of the $G$-orbit of $w_{i}$ is $\geq 2$.

We have $\gamma_{1}=2 \lambda_{1}-\lambda_{2}$. It follows from Corollary 2.9 that the equivariant section in $H^{0}\left(G \cdot x_{0}, \mathcal{N}_{X_{0}}\right)^{G}$ defined by $r_{1}$ extends to $X_{0}$.

We have $\gamma_{2}=\lambda_{2}-\lambda_{3}$. It follows from Corollary 2.9 that the equivariant section in $H^{0}\left(G \cdot x_{0}, \mathcal{N}_{X_{0}}\right)^{G}$ defined by $n r_{2,2}-q^{(2)}$ extends to $X_{0}$.

\section{REFERENCES}

[AB05] Valery Alexeev and Michel Brion, Moduli of affine schemes with reductive group action, J. Algebraic Geom. 14 (2005), no. 1, 83-117. MR MR2092127 (2006a:14017)

[ACF14] Roman Avdeev and Stéphanie Cupit-Foutou, On the irreducible components of moduli schemes for affine spherical varieties, arXiv:1406.1713v2 [math.AG], 2014. 
[BCF08] P. Bravi and S. Cupit-Foutou, Equivariant deformations of the affine multicone over a flag variety, Adv. Math. 217 (2008), no. 6, 2800-2821. MR MR2397467 (2009a:14061)

[Bou68] N. Bourbaki, Éléments de mathématique. Fasc. XXXIV. Groupes et algèbres de Lie. Chapitre IV: Groupes de Coxeter et systèmes de Tits. Chapitre V: Groupes engendrés par des réflexions. Chapitre VI: systèmes de racines, Actualités Scientifiques et Industrielles, No. 1337, Hermann, Paris, 1968. MR MR0240238 (39 \#1590)

[BR96] Chal Benson and Gail Ratcliff, A classification of multiplicity free actions, J. Algebra 181 (1996), no. 1, 152-186. MR MR1382030 (97c:14046)

[Bri10] Michel Brion, Introduction to actions of algebraic groups, Les cours du CIRM 1 (2010), no. 1, 1-22.

[Bri13] , Invariant Hilbert schemes, Handbook of moduli. Vol. I, Adv. Lect. Math. (ALM), vol. 24, Int. Press, Somerville, MA, 2013, pp. 64-117. MR 3184162

[BVS15] Paolo Bravi and Bart Van Steirteghem, The moduli scheme of affine spherical varieties with a free weight monoid, Int. Math. Res. Not. IMRN (2015), 44 pages, advance access, doi: 10.1093/imrn/rnv281.

[CLS11] David A. Cox, John B. Little, and Henry K. Schenck, Toric varieties, Graduate Studies in Mathematics, vol. 124, American Mathematical Society, Providence, RI, 2011. MR 2810322 (2012g:14094)

[FH91] William Fulton and Joe Harris, Representation theory, Graduate Texts in Mathematics, vol. 129, Springer-Verlag, New York, 1991, A first course, Readings in Mathematics. MR 1153249 (93a:20069)

[GS] Daniel R. Grayson and Michael E. Stillman, Macaulay2, a software system for research in algebraic geometry, available at http://www.math.uiuc.edu/Macaulay2/.

[GW09] Roe Goodman and Nolan R. Wallach, Symmetry, representations, and invariants, Graduate Texts in Mathematics, vol. 255, Springer, Dordrecht, 2009. MR 2522486 (2011a:20119)

[JR09] Sébastien Jansou and Nicolas Ressayre, Invariant deformations of orbit closures in $\mathfrak{s l}(n)$, Represent. Theory 13 (2009), 50-62. MR MR2485792

[Kno98] Friedrich Knop, Some remarks on multiplicity free spaces, Representation theories and algebraic geometry (Montreal, PQ, 1997), NATO Adv. Sci. Inst. Ser. C Math. Phys. Sci., vol. 514, Kluwer Acad. Publ., Dordrecht, 1998, pp. 301-317. MR MR1653036 (99i:20056)

[Lea98] Andrew S. Leahy, A classification of multiplicity free representations, J. Lie Theory 8 (1998), no. 2, 367-391. MR MR1650378 (2000g:22024)

[Los09] Ivan V. Losev, Proof of the Knop conjecture, Ann. Inst. Fourier (Grenoble) 59 (2009), no. 3, 1105-1134. MR MR2543664

[Lun01] D. Luna, Variétés sphériques de type A, Publ. Math. Inst. Hautes Études Sci. (2001), no. 94, 161-226. MR 1896179 (2003f:14056)

[PVS12] Stavros Argyrios Papadakis and Bart Van Steirteghem, Equivariant degenerations of spherical modules for groups of type A, Ann. Inst. Fourier (Grenoble) 62 (2012), no. 5, 1765-1809, extended version at arXiv:1008.0911v3 [math.AG]. MR 3025153

[Tim11] Dmitry A. Timashev, Homogeneous spaces and equivariant embeddings, Encyclopaedia of Mathematical Sciences, vol. 138, Springer, Heidelberg, 2011, Invariant Theory and Algebraic Transformation Groups, 8. MR 2797018 (2012e:14100)

[TY05] Patrice Tauvel and Rupert W. T. Yu, Lie algebras and algebraic groups, Springer Monographs in Mathematics, Springer-Verlag, Berlin, 2005. MR MR2146652 (2006c:17001)

[vLCL92] Marc A. A. van Leeuwen, Arjeh M. Cohen, and Bert Lisser, LiE, a package for Lie group computations, Computer Algebra Nederland, Amsterdam, 1992, available at http://www-math.univ-poitiers.fr/ maavl/LiE/.

[VP72] Ė. B. Vinberg and V. L. Popov, A certain class of quasihomogeneous affine varieties, Izv. Akad. Nauk SSSR Ser. Mat. 36 (1972), 749-764, English translation in Math. USSR Izv. 6 (1972), 743-758. MR MR0313260 (47 \#1815) 
Department of Mathematics, UniVersity of IOANNINA, 45110 IOANNINA, GREeCE

E-mail address: spapadak@cc.uoi.gr

Department of Mathematics, Medgar Evers College - City University of New York, 1650 BEDFORD AVE., BROOKLYN, NY 11225, USA

E-mail address: bartvs@mec. cuny . edu 